ADAPTIVE MULTILAYER OPTICE

FOR

EXTREME ULTRAVIOLET WAVELENGTHS
ADAPTIVE MULTILAYER OPTICS FOR

EXTREME ULTRAVIOLET

\section{WAVELENGTHS}




\section{ADAPTIVE MULTILAYER OPTICS FOR EXTREME ULTRAVIOLET WAVELENGTHS}

Muharrem Bayraktar 


\section{Ph.D. graduation committee}

Chairman \& secretary:

Prof. Dr. Ir. J.W.M. Hilgenkamp University of Twente

\section{Promotors:}

Prof. Dr. F. Bijkerk

University of Twente

Prof. Dr. K.J. Boller

University of Twente

\section{Members:}

Prof. Dr. Ing. A.J.H.M. Rijnders

University of Twente

Prof. Dr. D.J. Gravesteijn

Prof. Dr. B. Noheda

University of Twente \& NXP Semiconductors

Prof. Dr. C. Fallnich

University of Groningen

Prof. Dr. Ir. L. Abelmann

University of Münster \& University of Twente

University of Twente

The research presented in this thesis was carried out at the Laser Physics and Nonlinear Optics group, Department of Science and Technology, MESA+ Institute of Nanotechnology, University of Twente, P.O. Box 217, 7500 AE, Enschede, The Netherlands.

This research is supported by the Dutch Technology Foundation STW under project name "Smart Multilayer Interactive Optics for Lithography at Extreme Ultraviolet Wavelengths (SMILE)" with project number 10448.

Cover: Artist impression of wavefront correction using adaptive optics.

(C)Muharrem Bayraktar (2015)

Adaptive Multilayer Optics for Extreme Ultraviolet Wavelengths

Ph.D. thesis, University of Twente, Enschede, The Netherlands

Illustrated - With references - With summary in English and Dutch

ISBN: 978-90-365-3845-9

DOI: http://dx.doi.org/10.3990/1.9789036538459 


\section{ADAPTIVE MULTILAYER OPTICS FOR}

\section{EXTREME ULTRAVIOLET WAVELENGTHS}

\section{DISSERTATION}

to obtain

the degree of doctor at the University of Twente, on the authority of the rector magnificus, prof. dr. H. Brinksma,

on account of the decision of the graduation committee, to be publicly defended

on Thursday the $26^{\text {th }}$ of February 2015 at 16.45 .

by

\section{Muharrem Bayraktar}

born on the 15 December 1984

in Gerede, Turkey 
This dissertation has been approved by:

Promotors: $\quad$ Prof. Dr. F. Bijkerk

Prof. Dr. K.J. Boller 
This thesis is based on a patent and the following publications:

\section{- Chapter 2}

M. Bayraktar, F. A. van Goor, K.-J. Boller, F. Bijkerk, Spectral purification and infrared light recycling in extreme ultraviolet lithography sources, Optics Express 22, 8633 (2014).

\section{- Chapter 3}

M. Bayraktar, A. Chopra, F. Bijkerk, and G. Rijnders, Nanosheet controlled epitaxial growth of $\mathrm{PbZr}_{0.52} \mathrm{Ti}_{0.48} \mathrm{O}_{3}$ thin films on glass substrates, Applied Physics Letters 105, 132904 (2014).

\section{- Chapter 4}

M. Bayraktar, A. Chopra, F. Bijkerk, and G. Rijnders, Tuning of large piezoelectric response in nanosheet-buffered $\mathrm{PbZr}_{0.52} \mathrm{Ti}_{0.48} \mathrm{O}_{3}$ films on glass substrates, submitted.

\section{- Chapter 5}

M. Bayraktar, A. Chopra, G. Rijnders, K.-J. Boller, F. Bijkerk, Wavefront correction in the extreme ultraviolet wavelength range using piezoelectric thin films, Optics Express 22, 30623 (2014).

\section{- Chapter 6}

M. Bayraktar, W. A. Wessels, C. J. Lee, F. A. van Goor, G. Koster, G. Rijnders, and F. Bijkerk, Active multilayer mirrors for reflectance tuning at extreme ultraviolet (EUV) wavelengths, Journal of Physics D: Applied Physics 45, 494001 (2012).

\section{- Appendix}

A. Chopra, M. Bayraktar, F. Bijkerk, and G. Rijnders, Controlled growth of $\mathrm{PbZr}_{0.52} \mathrm{Ti}_{0.48} \mathrm{O}_{3}$ using nanosheet coated $\mathrm{Si}$ (001), submitted. 



\section{Contents}

1 Introduction 9

1.1 Motivation ..................... . . 9

1.2 Spectral purification and IR recycling in EUV sources . . . . . . 12

1.3 Basics of multilayer mirror optics . . . . . . . . . . . . . 13

1.4 Adaptive multilayer optics . . . . . . . . . . . . . . . . 15

1.4.1 Epitaxial growth of piezoelectric films on glass substrates . 16

1.4.2 Wavefront correction . . . . . . . . . . . . . . . . 17

1.4 .3 Reflectance tuning . . . . . . . . . . . . . . . . . . . . 19

1.4.4 Wavelength tuning . . . . . . . . . . . . . . . . 20

1.5 This thesis . . . . . . . . . . . . . . . . . . . 21

2 Spectral purification and infrared light recycling in EUV lithography sources 29

2.1 Introduction . . . . . . . . . . . . . . . . 30

2.2 Calculation of Fresnel zone pattern . . . . . . . . . . . . 31

2.3 Numerical results . . . . . . . . . . . . . . . . . . . . . . . . . 33

2.4 Conclusion . . . . . . . . . . . . . . . . 36

3 Nanosheet controlled epitaxial growth of $\mathrm{PbZr}_{0.52} \mathrm{Ti}_{0.48} \mathrm{O}_{3}$ thin films on glass $\quad 41$

3.1 Introduction . . . . . . . . . . . . . . . . . . 42

3.2 Experimental details . . . . . . . . . . . . . . . . . . 44 43

3.3 Results and discussion . . . . . . . . . . . . . . . . . . . . . 44

3.4 Conclusions . . . . . . . . . . . . . . . . . . . 46

4 Tuning of large piezoelectric response in nanosheet-buffered $\mathrm{PbZr}_{0.52} \mathrm{Ti}_{0.48} \mathrm{O}_{3}$ films $\quad \mathbf{5 5}$

4.1 Introduction . . . . . . . . . . . . . . . . . . 56

4.2 Experimental methods . . . . . . . . . . . . . . . . . 57

4.3 Results and discussion . . . . . . . . . . . . . . . . 58

4.4 Conclusions . . . . . . . . . . . . . . . . . . . . 64

5 Wavefront correction in the EUV wavelength range using piezoelectric thin films 71

5.1 Introduction . . . . . . . . . . . . . . . . 72

5.2 Growth of piezoelectric films on glass substrates . . . . . . . . . 73

5.3 Calculation of suitable pixel size . . . . . . . . . . . . . 74

5.4 Measurement of piezoelectric response . . . . . . . . . . 78

5.5 Summary and conclusion . . . . . . . . . . . . . . 79 
6 Active multilayer mirrors for reflectance tuning at EUV wavelengths 85

6.1 Introduction . . . . . . . . . . . . . . . 86

6.2 Active multilayer mirror structure . . . . . . . . . . . . . 86

6.3 Reflectance tuning range . . . . . . . . . . . . . . . . 87

6.4 Experimental results . . . . . . . . . . . . . . . . . . 90

6.5 Conclusions . . . . . . . . . . . . . . . . . . . 91

7 Valorization and outlook $\quad 95$

7.1 Enhancement of EUV source quality . . . . . . . . . . . . . . 96

7.2 Adaptive multilayer optics . . . . . . . . . . . . . . . . 96

7.2.1 Wavefront correction . . . . . . . . . . . . . . 97

7.2 .2 Reflectance tuning . . . . . . . . . . . . . . . . 98

7.2.3 Wavelength tuning . . . . . . . . . . . . . 98

7.2.4 Controlled epitaxial growth of piezoelectric films on glass substrates . . . . . . . . . . . . . . 99

A Wavelength tuning

B Controlled growth of $\mathrm{PbZr}_{0.52} \mathrm{Ti}_{0.48} \mathrm{O}_{3}$ using nanosheet coated $\mathrm{Si}(001) 107$

B.1 Introduction . . . . . . . . . . . . . . . . . . . . . 108

B.2 Experimental procedure . . . . . . . . . . . . . . . . . 109

B.3 Results and discussion . . . . . . . . . . . . . . . . . . 110

B.4 Conclusions . . . . . . . . . . . . . . . . . 113

$\begin{array}{lr}\text { C Zernike - Fringe polynomials } & 117\end{array}$

$\begin{array}{ll}\text { Summary } & 119\end{array}$

$\begin{array}{lr}\text { Nederlandse samenvatting } & 121\end{array}$

$\begin{array}{ll}\text { Acknowledgements } & 123\end{array}$ 


\section{Introduction}

\subsection{Motivation}

Imaging is closely related to our visual sense that provides us information. Thereby imaging has a vital role in understanding of what surrounds us, be it on the scale of the universe, or on the microscopic scale. With this role it is obvious that the amount of understanding that can be gained, for example about an object, enhances with the amount of information that can be gathered, which is done by imaging the object to more detail. In this sense imaging becomes more valuable as more details of the object can be resolved. Another feature of imaging is that it makes it possible to represent, regenerate or copy the information of an object at/to a different place than its original position. In this respect imaging enables transferring object information from one position to another. Again, this transfer is more valuable when the object is imaged to higher detail because more information is transferred. The combination of the resolving and the transfer features of imaging enables that collected information can either be widely spread, such as in displays, or that information can be condensed and arranged across extremely small areas. An example of the latter kind is photolithography in which the information about the structure of a complex electronic integrated circuit (IC) is imaged from a mask to a light-sensitive photoresist layer spread across a semiconductor wafer. Further processing steps, such as development of the photoresist and etching with liquids, plasmas or gases, can then permanently store, or "write" the image information in the form of a patterned surface or volume. This named technique, based on imaging using light to a semiconductor wafer, is called photolithography, literally meaning "writing on stone with light".

Due to high importance for the electronic devices, photolithography has undergone a dramatic advancement with regard to the amount of details that can be resolved. In the first versions applied in the early 1960s, circuit features with sizes around micrometers, which is about 1/100 of human hair thickness [1], could be patterned on the wafers [2-7]. Now, using current lithography tools, it is possible to pattern ICs with feature sizes as small as $32 \mathrm{~nm}$ [6], which is only a few hundred atomic diameters. This miniaturization enables fabrication of more compact, more powerful and more energy efficient electronic devices. These advantages of miniaturized ICs explain also the desire of the semiconductor industry to further reduce the chip feature sizes.

It is clear that the users of the chips with small feature sizes, built into communication devices or data storage and processing devices, demand that miniaturization keeps progressing as long and as fast as possible. However current lithography tools are approaching their physical limit that is set by nature. The resolution in imaging which determines the minimum feature size in lithography, 
is limited by the light wavelength used. The effect that underlies this limitation is called diffraction, which is the fundamental property of waves to spread out also transversely during propagation. A way to reduce the undesired spread, which would correspond to a loss of information, is to use shorter wavelengths. The shortest wavelength presently used is in the ultraviolet (UV) range at $193 \mathrm{~nm}$ and the next wavelength range that is considered is the extreme ultraviolet (EUV) around $13.5 \mathrm{~nm}$. Due to this extremely small wavelength, EUV lithography has been capable to fabricate sub- $22 \mathrm{~nm}$ features [6-8]. Even smaller wavelength at $6.7 \mathrm{~nm}$ is anticipated for further miniaturization of the chips in the future [9-11].

Yet, with the clear need to use extremely short wavelength in the EUV wavelength range, the properties of radiation in this range represent some significant challenges. The reason is that some of the most basic and well-known concepts for imaging, well-working in the visible, tend to fail at increasingly shorter wavelength. The reason is that the primary inner-shell resonance lines of almost all chemical elements lie in the EUV range, therefore, almost all materials are highly absorptive in this wavelength range. Essentially, the transmission of EUV light through any solid or liquid material or optical component becomes zero, except for extremely thin films with sub-micrometer thickness [12]. This extremely high strength of absorption basically excludes the usage of any transmissive optics, leaving reflective optical components the only viable choice. Nevertheless, without further precautions, also reflective optics perform poorly. The reason is that in the EUV range, the reflection from a single interface or surface is much too weak to be useful, typically only below $1 \%$. This marked difference with the visible and infrared range is due to the fact that in the EUV range the refractive index of all materials are close to unity, which diminishes the index contrast at interfaces to almost zero. To achieve a high reflectance in spite of low contrast, EUV mirrors need to comprise multiple layers to provide multiple interfaces, where the partial reflection from the interfaces is coherently added at selected wavelengths and angles of reflection. At a wavelength of $13.5 \mathrm{~nm}$ the current record in reflectivity amounts to about $70 \%$, being close to what is theoretically possible, and has been achieved using 100 alternating layers of Mo and Si (i.e. 50 so-called Mo/Si bilayers) [13]. Such multilayer mirrors are the prime optical components for EUV, however, there are constraints for using them in high numerical aperture lithography systems, which is due their strong dispersion. Because based on interference, EUV multilayer mirrors reflect only within a smaller range of incident angles as will be explained in 1.3. To nevertheless collect or image light over a large field of view, comprising a large range of incident angles, multiple mirrors are required. A typical example, in this case comprising 11 reflective components, is shown in Fig. 1.1 [6]. The EUV light is in this case generated in a plasma via a high power infrared laser $\left(\mathrm{CO}_{2}\right.$ drive laser $)$ from a tin (Sn) target. The emitted EUV light is collected by a first mirror (collector) and then directed by four subsequent mirrors to illuminate the so-called reticle, which is the object to be imaged. Imaging onto the photoresist layer on a wafer is performed with another set of six multilayer mirrors.

The presented EUV lithography setup can pattern extremely fine features, i.e., below $22 \mathrm{~nm}$ [6]. Yet, these benefits come together with two challenges in 


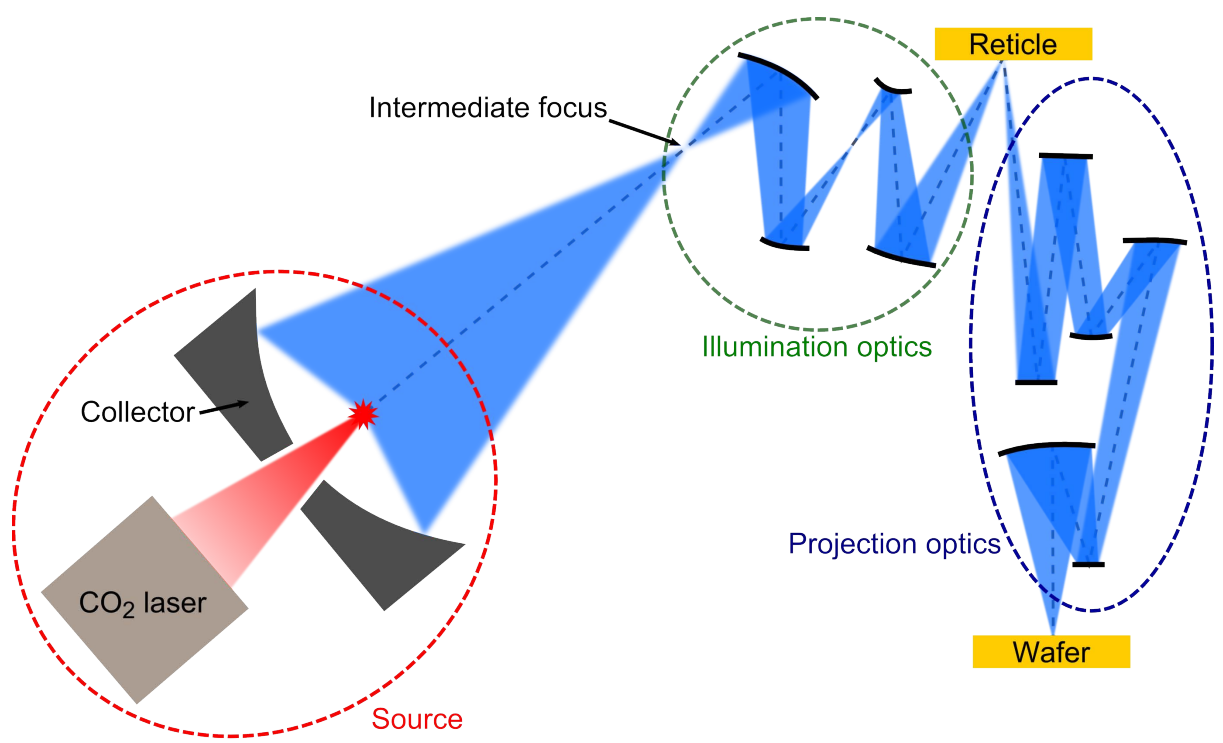

Figure 1.1: An all-reflective EUV imaging system used in EUV lithography [6]. A high power $\mathrm{CO}_{2}$ laser is generating a plasma that is intensively emitting EUV light. This EUV light is directed by the collector and illumination mirrors to the reticle. The reticle is then imaged to the wafer by the projection mirrors.

the EUV lithography system that need to be carefully taken into account. The first challenge lies in the source and is posed by an undesired partial reflection of the IR drive laser light from the Sn plasma [14, 15]. The reflected IR light is directed by the collector mirror, together with the EUV light, through the entire lithography system. The problems caused thereby are twofold. First the IR light is lost for the plasma generation and, second, the IR light causes an unfavorable heating of the photoresist materials used in the lithography process, which can severely lower the quality of imaging. The second challenge is found in the multilayer mirrors due to that part of the EUV light which is not reflected but absorbed. The resulting heat load and temperature changes in the multilayer mirror [16-20] can lead to a distortion of the reflected wavefronts, a degradation of the mirror reflectance and a deviation of the reflectance curve from the required wavelength band. All these challenges are threatening the optical resolution of the lithography tool from reaching its full potential.

In this thesis we developed novel approaches for EUV optical components in order to meet the named challenges in EUV lithography. The first approach is a novel design for an EUV collector mirror that enhances the source quality by spectrally purifying the EUV light and that simultaneously recycles IR drive laser light back into the plasma. In the second approach a new class of multilayer optics with adaptive features was investigated. These adaptive multilayer optics are based on piezoelectric layers integrated into the EUV mirrors that 
can be adjusted externally. The developed adaptive optics can be applied for three different tasks, i.e., correct wavefront distortions, reflectance degradations or wavelength deviations. The integration of piezoelectric layers into multilayer mirrors necessitates growing high quality crystalline piezoelectric films on glass substrates, i.e., on amorphous structures. In this thesis we achieved the first epitaxial growth of piezoelectric films on glass substrates, and we thereby achieved the highest known piezoelectric response. Importantly, this response is in a magnitude relevant for the range where wavefront distortions can occur in the EUV wavelengths. In the next sections, we first explain in more detail the developed collector mirror design, then give a brief overview on basics of multilayer mirror optics and explain the developed variants of adaptive optical components as based on epitaxial piezoelectric film growth on glass substrates.

\subsection{Spectral purification and IR recycling in EUV sources}

The presented source configuration can collect significant EUV powers, but there are two challenges due to partial reflection of the IR drive laser light from the plasma $[14,15]$. First, the reflected IR light is lost for the plasma generation process. Second, the IR light is directed to the most undesired location, the exit of the source at the intermediate focus, from where it can follow the same path as the EUV light. This is highly undesired because the high power of the IR light causes heating of the photoresist material used in the lithography process.

Potential solutions to prevent this undesired IR light from reaching the photoresist have been proposed based on membranes, absorbing gases and gratings [2130]. Membranes and gaseous filters may impose thermally induced distortions to the EUV light and the grating approaches do not recycle any reflected IR light. Recycling of the lost IR light has not been addressed up to now.

In this thesis we present a collector mirror design that solves both problems simultaneously. The presented design is based on patterning a Fresnel zone plate (FZP) at the collector mirror surface. Fresnel zone plates are focusing diffractive structures and since the focusing is based on diffraction, it is strongly chromatic, i.e. wavelength dependent. As schematically shown in Fig. 1.2 a FZP is comprised of concentric rings with alternating phases. The phase and radius of the rings can be selected, i.e. matched, to focus a specific wavelength $\lambda_{1}$ to a chosen point $F$ in Fig. 1.2. The rest of the wavelengths, especially if they are largely separated from the focusing wavelength, are minimally affected and reflected back due to mismatch between the structure of the FZP and the wavelengths.

There are two advantages of FZPs for our purpose as such their focal distances can be adjusted by changing the surface structure and they can be integrated onto curved mirrors. When integrated on a curved mirror FZP introduces, to a specific wavelength, an extra focusing power that can be adjusted by the surface structure. In our design a FZP is patterned on the collector mirror surface to divert the IR light from the exit of the source and focus back to the plasma for further heating of the plasma. Diversion of the IR light from the exit of the 


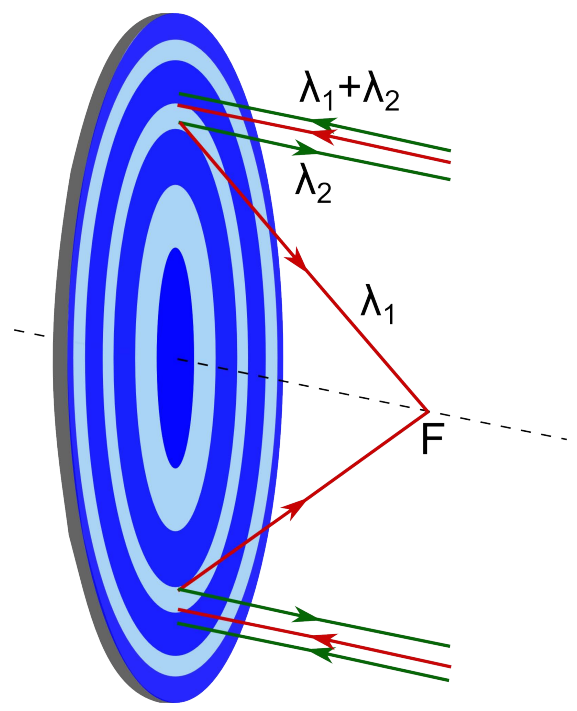

Figure 1.2: A Fresnel zone plate (FZP) is comprised of concentric rings with alternating phases as shown with dark and light blue colors. The phase and radius of the FZP rings are selected to focus a specific wavelength $\lambda_{1}$ to a desired distance $F$. The other wavelength $\lambda_{2}$ that is not matching the phase and ring radius of the FZP is reflected back.

source effectively results in a strong purification of the EUV light at the exit of the source. This design achieves a purification factor of four orders of magnitude and at the same time recycles approximately $35 \%$ of the otherwise wasted IR laser light. Since the EUV wavelength is largely, orders of magnitude, smaller than the IR wavelength, the effect of the FZP on the EUV light is negligibly small.

\subsection{Basics of multilayer mirror optics}

A multilayer mirror consists of at least two different materials as shown in Fig. 1.3(a) that have a difference in their refractive indices. In the EUV wavelength range, where all materials are strongly absorptive and have indices very close to unity, it is convenient to express the complex refractive index via the socalled refraction coefficient $\delta$ and the absorption coefficient $\beta$ as $n=1-\delta+i \beta$, where both $\delta$ and $\beta$ are much smaller than unity. At the interface between two materials, partial Fresnel reflection occurs due to the refractive index difference [31]. In order to have maximum partial reflection in a specific wavelength range, two materials are chosen that offer a larger difference in their refraction coefficients, while ensuring lower absorption coefficients. The materials with high and low refraction coefficients are usually referred to as the absorber and spacer layers that are shown with dark and light blue in Fig. 1.3(a), respectively. The 
partially reflected beams add up constructively when the bilayer period, $d$, is chosen according to the generalized Bragg condition [12]:

$$
m \lambda=2 d \sin \theta \sqrt{1-\frac{4 \bar{\delta} d^{2}}{m^{2} \lambda^{2}}}
$$

where $m$ is the diffraction order, $\lambda$ is the wavelength, $\theta$ is the grazing angle of incidence and $\bar{\delta}$ is the thickness weighted average of the refraction coefficients in a bilayer period.

Considering the near-unity indices at EUV wavelengths, the period of the multilayers equals approximately half of the wavelength. As a result, an individual layer is extremely thin, in the nanometer range, i.e., only 10-20 atoms thick, such that fabrication is not straightforward at all. There has been an in-depth research and progress in the last 20 years in achieving reflectance values close to the theoretical maximum. This progress has been enabled mostly by understanding the physical processes taking place during, and also after, layer deposition, i.e., also during usage of the mirrors. This understanding includes critical nanoscopic parameters such as the roughness at the bilayer interface, the composition and morphology of the layers, and the thickness of the interfacial layer that inevitably forms during deposition. All of these parameters have been optimized so far using techniques like ion-smoothing, and using capping layers or barrier layers that control or prevent interfacial layer formation. By now, these techniques have matured for the $13.5 \mathrm{~nm}$ wavelength, as can be seen from reflectance values that are close to the theoretical values and can be achieved reproducibly. In Fig. 1.3(b) the measured high reflectance at $13.5 \mathrm{~nm}$, a record value of $70 \%$, is shown as achieved with a Mo/Si multilayer mirror [13].
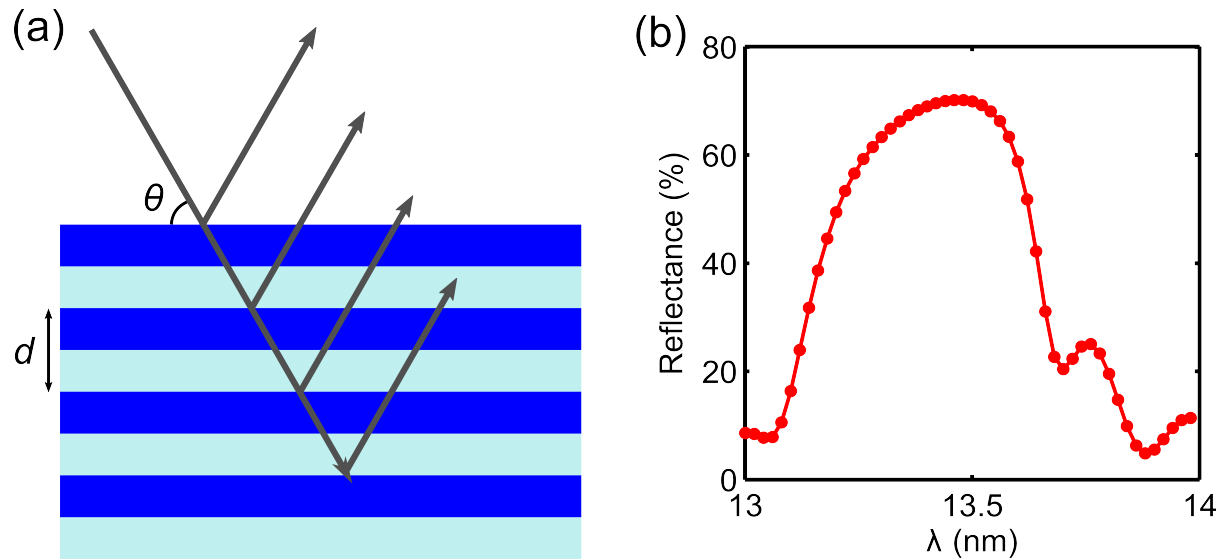

Figure 1.3: (a) Multilayer mirror (b) The measured high reflectance at $13.5 \mathrm{~nm}$, a record value of $70 \%$, achieved with a $\mathrm{Mo} / \mathrm{Si}$ multilayer mirror [13].

The less than full reflectance of EUV mirrors has an important implication. The fraction of the incoming light absorbed in the multilayer, combined with the 
high average powers required in applications such as free electron laser research or EUV lithography, creates a significant heat load on the optical components [1620]. The associated temperature changes could lead to a change in the mirror substrates or the multilayer coatings that in turn result in distortion of the reflected wavefronts, degradation of the mirror reflectance or a spectral shift of the maximum of the reflectance spectrum, away from the desired wavelength. These distortions, degradations or deviations can significantly reduce the optical resolution and performance of the overall lithography system. Obviously, there is a strong need for optical components that could compensate for such effects.

In this thesis we introduce novel types of optical components that allow adaptation of their dimensions by external control and we investigate how they compensate wavefront distortions, degradations and spectral deviations. These optics are based on piezoelectric films integrated to the multilayer mirrors. We call this new class of optical components adaptive multilayer optics.

\subsection{Adaptive multilayer optics}

The desired adaptations can be accomplished using piezoelectric materials integrated into the multilayer mirror stack as shown in Fig. 1.4. In Fig. 1.4(a) a piezoelectric layer is situated beneath the multilayer mirror stack. This scheme allows moving the complete multilayer mirror stack perpendicular to the substrate surface. The reflectance spectrum does not change but the reflected light is phase-shifted. Thereby the scheme can be used for correcting wavefront distortions or shaping the wavefront as desired. In the second scheme, shown in Fig. 1.4(b), the piezoelectric layer is integrated between two multilayer mirror stacks. In this scheme the incident waves will be reflected partially from the upper and lower multilayer stacks and these reflections will interfere. The degree of interference can be controlled by adjusting the thickness of the piezoelectric layer as in a Fabry-Perot etalon or a microcavity. Depending on the interference, the total reflectance at a selected wavelength can be tuned. In Fig. 1.4(c) piezoelectric layers are integrated into each bilayer. This scheme can be used to shift the complete reflectance curve to different wavelengths or to tune the complete wavelength response of the multilayer.

In these adaptive multilayers the ability to integrate the piezoelectric films into the multilayer mirrors is essential. The central problem here is that piezoelectric films need to consist of crystalline materials, for achieving a sufficient stroke (maximal change in thickness), in contrast to the template systems, like optics substrates and multilayers, which need to be made of amorphous materials. This necessitates to realize the growth of crystalline piezoelectric films on amorphous templates. Though growth of crystalline piezoelectric films on crystalline substrates is a well-studied and established field, the knowledge on growing high quality crystalline piezoelectric films on glass substrates is limited. The reported piezoelectric films on glass substrates are mostly polycrystalline which limits their piezoelectric response to quite low values compared to epitaxially grown piezoelectric films on crystalline substrates. For example, the reported piezoelectric 

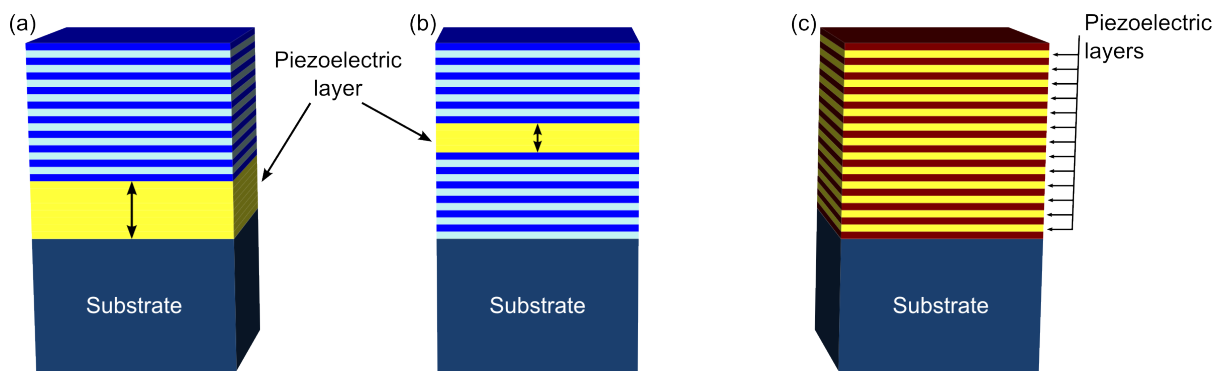

Figure 1.4: (a) Wavefront correction with a piezoelectric layer (yellow) integrated beneath the multilayer mirror (b) Reflectance tuning with a piezoelectric layer deposited between two multilayer mirror stacks (c) Wavelength tuning with piezoelectric layers integrated at each period of the multilayer mirror.

response on glass is only $30 \mathrm{pm} / \mathrm{V}$ [32], to be compared to $330 \mathrm{pm} / \mathrm{V}$ for epitaxial films $[33,34]$. Therefore the ability to grow high quality, if possible epitaxial, piezoelectric films on glass substrates is absolutely essential for our adaptive multilayer optics, and an according success would be of highest importance also for many other applications such as data storage and electronic displays [35-39].

\subsubsection{Epitaxial growth of piezoelectric films on glass substrates}

In this thesis we report on the first growth of locally epitaxial piezoelectric films on glass substrates. The transition from the amorphous structure of the substrate to epitaxial structure of the piezoelectric film was achieved by using crystalline nanosheets deposited on the substrate. The composition of the piezoelectric films was selected as $\mathrm{PbZr}_{0.52} \mathrm{Ti}_{0.48} \mathrm{O}_{3}$ (PZT), a material which is known to have the highest piezoelectric coefficient. The deposition of the piezoelectric layer was performed using pulsed laser deposition (PLD) that is a proven technique to grow high quality piezoelectric films. In the first attempts with low deposition rate, a high piezoelectric coefficient of $98 \mathrm{pm} / \mathrm{V}$ was measured for the grown piezoelectric films.

The piezoelectric response in thin films is known to be reduced compared to their bulk values due to clamping by the substrate. This clamping effect typically limits the piezoelectric response in a thin film to half or one third of the bulk piezoelectric response [40]. We report on minimization of the clamping effect to enhance the piezoelectric response. The PZT films that we deposited at the highest repetition rate has the lowest clamping effect and the highest piezoelectric response, namely $280 \mathrm{pm} / \mathrm{V}$. This piezoelectric response is, to the best of our knowledge, the highest value reported on glass substrates.

These adaptive multilayers represent a novel class of optics that has not been described or fabricated before. Due to this absolute novelty, there is a risk of failures in fabrication, or there can be unforeseen limitations but, more importantly, there is a huge potential for substantial innovation and breakthrough. In this 
thesis we have explored all of the three adaptive multilayer schemes. The first scheme aiming for wavefront correction, Fig. 1.4(a), was experimentally demonstrated. The second scheme for reflectance tuning, Fig. 1.4(b), was theoretically explored and though the complete stack has not yet been fabricated, feasibility of the critical parameters was experimentally demonstrated. The third scheme aiming at tuning of the wavelength response has thus far been explored only theoretically. In the next three sections, the relevant literature reviews and the key findings for each scheme are presented.

\subsubsection{Wavefront correction}

Wavefront correction is a well-known technique in the infrared and visible wavelengths. In modern imaging systems used in microscopy, astronomy or vision science wavefront correction, typically done using deformable mirrors, is indispensable to achieve diffraction limited resolution [41-44]. In order to have a laterally fine correction, the deformable mirror substrates are usually made from thin membranes with reflective coatings. The thin membrane/substrate is deformed by an array of actuators placed at the back side. Several deformable mirrors with piezoelectric, magnetic or electrostatic actuators are available for these relatively long wavelengths [45-53].

In contrast, the wavefront correction approach needs to be quite different in the EUV range, where wavelengths are approximately two orders of magnitude shorter. This difference translates into a much higher requirement for mechanical and thermal stability in the sub-nanometer range. For satisfying the mechanical stability requirement, the substrates need to be several centimeters thick. In order to have thermal stability at the same time, the substrates have to be composed of glasses with a low coefficient of thermal expansion such as Fused Silica, Zerodur or Ultra Low Expansion (ULE) glass. Imposing these requirements excludes the usage of bending type deformable mirrors developed for grazing incidence optics in soft x-ray telescopes [54-61]. The two approaches which have been considered in the literature so far, and their associated drawbacks compared to our approach are explained as follows.

The first approach described in the literature is based on an array of thermomechanical actuators/pillars that are glued to the back side of a Zerodur mirror substrate as shown in Fig. 1.5(a-b) $[62,63]$. In this approach the complete thick substrate has to be bent, so there is a strong coupling between neighboring actuators, as shown in Fig. 1.5(a). In order to reduce the coupling between neighboring actuators and have a more localized surface deformation, a thin back plate is used as shown in Fig. 1.5(b). Since the actuation is based on expansion of the actuators using thermal heating, the resulting actuation is slow, i.e., $2 \mathrm{~nm} / \mathrm{min}[62,63]$.

The second approach is also based on thermal actuation. In this approach a thick Fused Silica substrate is heated from back using a spatially extended heat source as shown in Fig. 1.5(c) [20]. The heat source used in this approach is a high power light source that is spatially structured using an adjustable mask. The heat is absorbed by an absorptive coating deposited at the front surface of the substrate to induce a surface modification. This approach can use the already 
available thick substrates due to its non-contact nature. This approach is indeed accurate but the actuation is slow, $0.1 \mathrm{~nm} / \mathrm{min}$, and the lateral resolution of the actuation is limited to centimeters due to heat conduction [20].
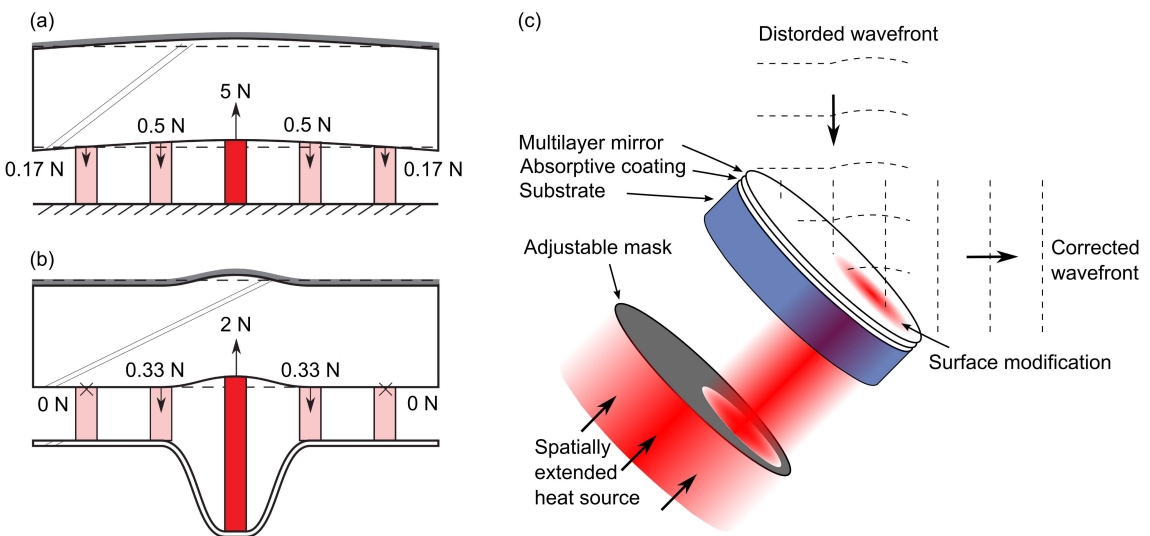

Figure 1.5: (a-b) Wavefront correction by thermo-mechanical actuators/pillars glued at the back side of the substrate $[62,63]$. Schematic cross-sections of the three dimensional finite element analysis. Actuators are laterally positioned in two co-centric hexagons, such that the actuator at the center is surrounded by six actuators positioned in a hexagon shape and these seven actuators are surrounded by another 12 actuators again positioned in a hexagon shape. (a) When the actuators are fixed from the bottom, there is a strong coupling between neighboring actuators. The force exerted by the energized actuator at the center is balanced by the forces at the surrounding actuators, i.e., 5 Newton $(\mathrm{N}) \approx 6 \times 0.5 \mathrm{~N}+12 \times 0.17 \mathrm{~N}$. (b) The coupling can be reduced using a thin back plate. The force balance is given by $2 \mathrm{~N} \approx 6 \times 0.33 \mathrm{~N}$. (c) Wavefront correction using structured back-illumination with spatially extended heat source [20]. The illumination is structured using an adjustable mask to create/compensate thermal gradients on the substrate surface. The heat is absorbed by the absorptive coating deposited at the front surface of the substrate.

Our wavefront corrector approach shown in Fig. 1.4(a) is a simplified schematic drawing of the complete stack, whereas in a practical case the piezoelectric films additionally have electrodes for applying a voltage and buffer layers for ensuring epitaxial growth, as shown in Fig. 1.6. Figure 1.6(a) shows a schematic drawing of the piezoelectric film stack with electrodes and the nanosheet buffer layer. In Fig. 1.6(b) the scanning electrode microscope (SEM) image of the deposited film stack is shown. The SEM image reveals a highly columnar structure as an indication of the high quality growth. Having assured a high quality growth is the first step towards wavefront correction with the developed piezoelectric films. The next step is to make sure that the developed films can achieve sufficient surface displacements needed for applications in the EUV wavelengths. Yet, the wavefront correction needs to have a laterally fine resolution.

In this thesis we demonstrate that the developed piezoelectric films can achieve a large surface displacement up to $25 \mathrm{~nm}$ with the required fine resolution. This 
surface displacement can clearly be named large, because it compares favorably with the required value of $3.4 \mathrm{~nm}$, which is a value calculated in [20] considering the high power operation of a EUV lithography tool and also corresponds to quarter wavelength for $13.5 \mathrm{~nm}$. The developed piezoelectric films show a piezoelectric response of $250 \mathrm{pm} / \mathrm{V}$ which enables achieving the required surface displacement with a moderate voltage.
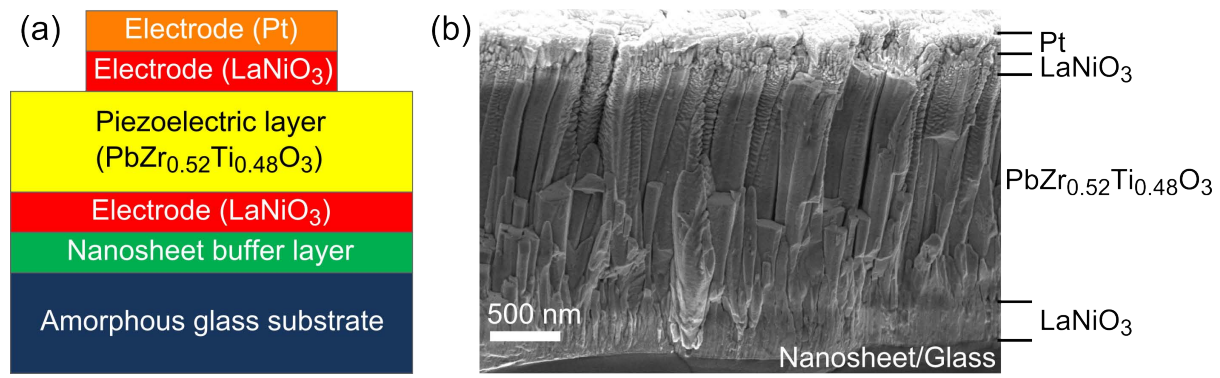

Figure 1.6: (a) The piezoelectric film stack. (b) The scanning electron microscope (SEM) image of the deposited film stack.

The main characteristics of the two wavefront correction approaches and our piezoelectric thin film approach are compared in Table 1.1. The piezoelectric approach offers fast actuation speed and high spatial resolution. Considering our piezoelectric thin films, a stroke of $25 \mathrm{~nm}$ could be achieved in sub-seconds, resulting a conservative prediction of the speed as $25 \mathrm{~nm} / \mathrm{s}$ or $1500 \mathrm{~nm} / \mathrm{min}$. The response of piezoelectric materials is known to suffer from hysteresis, however, this is much weaker in piezoelectric thin films than in commonly known bulk piezoelectric materials [64-66]. The hysteresis can be decreased even more by staying on the same side of the hysteresis loop or by other control methods [67, $68]$.

\subsubsection{Reflectance tuning}

The second adaptive multilayer mirror structure explored is shown Fig. 1.4(b). In this adaptive mirror, the piezoelectric film is integrated between two stacks of multilayers to control the phase difference between the beams reflected from the upper and lower multilayer stack. By controlling the phase difference, the interference between the beams can be tuned from destructive to constructive and the total reflectance can be increased, or vice versa. This scheme can be used to have a mirror with varying reflectance across its surface after patterning the mirror into separate regions. Such a mirror can be used to homogenize a non-homogeneous illumination profile.

A detailed drawing of the complete multilayer mirror stack is shown in Fig. 1.7. In this scheme, the piezoelectric layer is equipped with electrodes and the buffer layer similar to the wavefront correction case. In contrast, the piezoelectric material is in the optical path, hence its optical properties such as the surface 
Table 1.1: Wavefront correction approaches for the EUV wavelength range

\begin{tabular}{lll}
\hline & Wavefront correction approach & Characteristics \\
\hline Ravensbergen & Thermo-mechanical actuation & - Low speed \\
{$[62,63]$} & & $(2 \mathrm{~nm} / \mathrm{min})$ \\
Saathof $[20]$ & Structured back illumination & - Low speed \\
& & $(0.1 \mathrm{~nm} / \mathrm{min})$ \\
& - Low spatial resolution \\
This thesis & Piezoelectric thin films & - High speed \\
& & $(1500 \mathrm{~nm} / \mathrm{min})$ \\
& & - High spatial resolution \\
& - Hysteresis behavior may \\
& & require correction
\end{tabular}

roughness and absorption introduced by the piezoelectric layer are important. To have low surface roughness, piezoelectric films with $\mathrm{PbZr}_{0.20} \mathrm{Ti}_{0.80} \mathrm{O}_{3}$ composition were explored for this structure. Modeling of the reflectance of these mirrors show that the optimum thickness of the piezoelectric layer is in the range of $20 \mathrm{~nm}$. We confirmed a piezoelectric response of $60 \mathrm{pm} / \mathrm{V}$ for the developed $\mathrm{PbZr}_{0.20} \mathrm{Ti}_{0.80} \mathrm{O}_{3}$ thin films. The developed films have a quite low surface roughness of $0.3 \mathrm{~nm}$, comparable to the roughness of the underlying substrate of 0.2 $\mathrm{nm}$. With these piezoelectric films, a reflectance tuning range in the order of the total strain, i.e. $\sim 1 \%$ is achievable.

\subsubsection{Wavelength tuning}

The third adaptive multilayer scheme may have a high applicability too, yet it represents still an effort in development. In this scheme the piezoelectric material is integrated into every bilayer as shown in Fig. 1.4(c) to have control over the spectral response of the multilayer mirror. Since one of the materials in the multilayer stack is replaced with a piezoelectric material, the reflectance becomes an important parameter to be investigated. In Appendix A, we calculated the reflectance of these adaptive multilayers for two wavelengths that are of main interest for EUV lithography, $13.5 \mathrm{~nm}$ and $6.7 \mathrm{~nm}$, In the multilayer stack, the thickness of each layer is approximately a quarter of the wavelength. For $6.7 \mathrm{~nm}$ wavelength thickness of a single layer is below $2 \mathrm{~nm}$. With such small thicknesses and the need for crystallinity for the piezoelectric layer, the multilayer stack should likely be grown completely from crystalline materials. Here we considered crystalline perovskite materials that are known to have piezoelectric response at such thicknesses and studied extensively for their remarkably high piezoelectric response [69]. In the reflectance calculations, we kept the weakly absorbing Si 


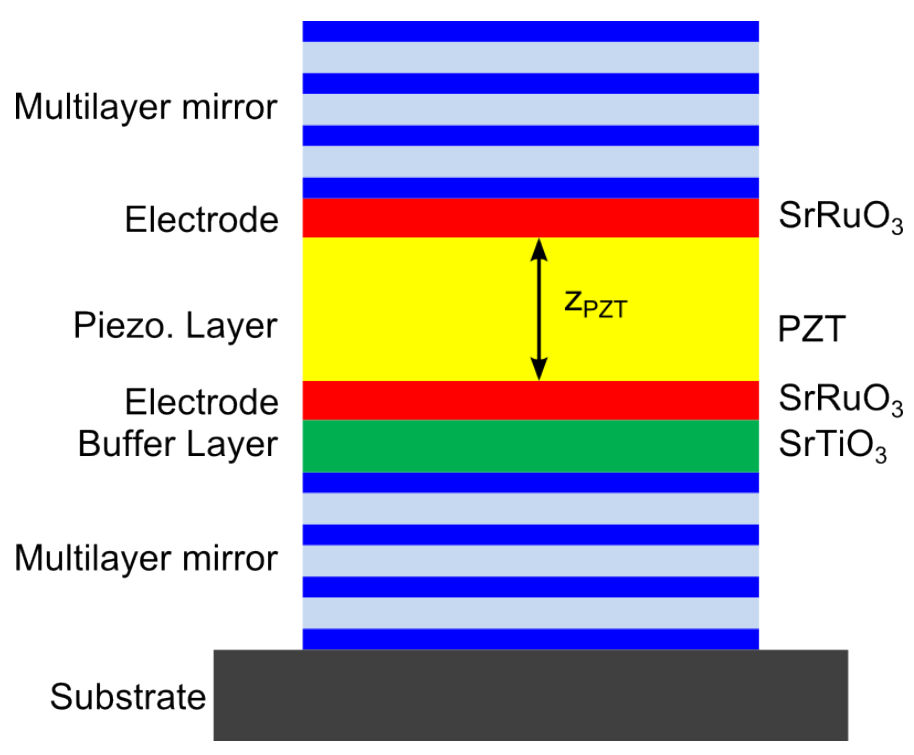

Figure 1.7: Detailed drawing of the reflectance tuning film stack. The piezoelectric layer, electrodes and the buffer layer are integrated between two multilayer mirror stacks.

and B layers for $13.5 \mathrm{~nm}$ and $6.7 \mathrm{~nm}$ respectively and paired them with perovskite materials taken from a crystallography database [70-72]. The multilayer stacks that have reflectance values above $40 \%$ are presented in Table 1.2. It can be seen that the reflectance of the adaptive mirrors reaches considerable reflectance values, up to $49 \%$ and $53.5 \%$ at wavelengths $13.5 \mathrm{~nm}$ and $6.7 \mathrm{~nm}$, respectively. These reflectance values are somewhat lower than the maximum reflectance values of $70 \%$ and $57 \%$ achieved at $13.5 \mathrm{~nm}$ and $6.7 \mathrm{~nm}$, respectively. The experimental realization of this adaptive mirror scheme needs further investigation.

\subsection{This thesis}

The motivation of this thesis can be briefly described as enhancing the performance of imaging at EUV wavelengths. Though the motivation is optical, the topics covered in this thesis spans a much wider field including piezoelectric materials, thin film growth and mechanics in addition to optics. In view of this multidisciplinary nature, we aimed on each chapter being self-contained instead of assembling all background into a separate mixed-theory chapter. For the sake of conciseness some of the supporting information and derivation of the basic equations are presented in the appendices. The thesis is organized as follows:

- Chapter 2 presents a collector mirror design for the laser produced plasma light sources of the EUV lithography tools. This collector mirror design comprises of a Fresnel zone plate patterned into the surface of the collector mirror to spectrally purify the EUV light from the IR light at the exit of the source and 
Table 1.2: WReflectance of spectrally adaptive multilayer mirrors at $13.5 \mathrm{~nm}$ and 6.7 $\mathrm{nm}$ wavelengths.

\begin{tabular}{|c|c|c|c|}
\hline $\begin{array}{l}\text { Wavelength } \\
(\mathrm{nm})\end{array}$ & Absorber & $\begin{array}{l}\text { Density } \\
\left(\mathrm{g} / \mathrm{cm}^{3}\right)\end{array}$ & $\begin{array}{l}\text { Reflectance } \\
\%\end{array}$ \\
\hline 13.5 & $\mathrm{SrCa}_{0.33} \mathrm{Nb}_{0.67} \mathrm{O}_{3}$ & 5.09 & 49.0 \\
\hline (Spacer layer: Si, & $\mathrm{CaIrO}_{3}$ & 8.62 & 46.0 \\
\hline \multirow[t]{11}{*}{ Density: $2.33 \mathrm{~g} / \mathrm{cm}^{3}$ ) } & $\mathrm{YMnO}_{3}$ & 5.15 & 45.8 \\
\hline & $K_{0.3} \mathrm{Na}_{0.7} \mathrm{NbO}_{3}$ & 4.50 & 43.7 \\
\hline & $\mathrm{Mn}_{2} \mathrm{O}_{3}$ & 5.03 & 43.5 \\
\hline & $K_{0.05} \mathrm{Na}_{0.95} \mathrm{NbO}_{3}$ & 4.51 & 42.1 \\
\hline & $L i_{0.02} \mathrm{Na}_{0.98} \mathrm{NbO}$ & 4.61 & 42.1 \\
\hline & $\mathrm{CaCO}_{3}$ & 2.57 & 41.4 \\
\hline & $\mathrm{PbZn}_{0.31} N b_{0.61} T i_{0.08} \mathrm{O}_{3}$ & 8.39 & 40.9 \\
\hline & $\mathrm{Tl}_{2} \mathrm{O}_{3}$ & 10.3 & 40.5 \\
\hline & $\mathrm{PbH}_{0.4} \mathrm{Ti}_{0.6} \mathrm{O}_{3}$ & 9.67 & 40.4 \\
\hline & $\mathrm{FeMnO}_{3}$ & 5.16 & 40.2 \\
\hline & $\mathrm{PbVO}_{3}$ & 7.54 & 40.0 \\
\hline 6.7 & $L a_{2} \mathrm{O}_{3}$ & 6.61 & 53.5 \\
\hline (Spacer layer: B, & $\mathrm{Ce}_{2} \mathrm{O}_{3}$ & 6.90 & 45.4 \\
\hline Density: $2.37 \mathrm{~g} / \mathrm{cm}^{3}$ ) & $\mathrm{Pr}_{2} \mathrm{O}_{3}$ & 7.11 & 41.5 \\
\hline
\end{tabular}

at the same time focus the IR light to the plasma to enhance the IR-to-EUV conversion. Using diffraction calculations, it is shown that a strong purification of the EUV light by four orders of magnitude and recycling of the IR light by $35 \%$ can be achieved.

- In chapter 3, we demonstrate the growth of crystalline piezoelectric films on amorphous glass substrates with control on the growth orientation. The transition from amorphous phase to crystalline phase was promoted by using crystalline nanosheet as the buffer layers on the glass substrates. The nanosheet were prepared chemically in crystalline form and transferred to the substrate using Langmuir-Blodgett deposition. The growth orientation was controlled by selecting different nanosheets and locally epitaxial growth has been achieved. Characterization of the microstructure, electrical and piezoelectric properties of the films are presented.

- In chapter 4, enhancement of the piezoelectric response in thin films is demonstrated. In thin films the piezoelectric response is reduced compared to their bulk counterparts due to clamping effect induced by the substrate. We control the degree of clamping by tuning the density of the piezoelectric film. The density 
was varied by the repetition rate of the deposition or using a buffer layer [73]. We achieved a record high piezoelectric response of $280 \mathrm{pm} / \mathrm{V}$ for the optimized films.

- Chapter 5 continues with the demonstration of the wavefront correction that can be achieved with the developed piezoelectric films. Relevant wavefront distortions are considered in order to evaluate the wavefront correction performance of the presented approach. It is demonstrated that the developed films can achieve a stroke of $25 \mathrm{~nm}$ and perform the desired wavefront corrections with relatively low voltage of $13.5 \mathrm{~V}$.

- In chapter 6 , a novel multilayer mirror design is presented in which an adaptive piezoelectric layer is placed between two standard multilayer mirrors similar to an etalon structure. Using such a mirror design the total reflectance can be tuned by changing the thickness of the piezoelectric layer. It was experimentally verified that the piezoelectric films can be made with a high smoothness that satisfies the requirement of the EUV lithography, and at the same time shows an acceptable piezoelectric response of $60 \mathrm{pm} / \mathrm{V}$.

- In chapter 7 , we summarize the findings of the thesis and give an outlook towards valorization of the results, i.e., we consider potential utilization of the results in a variety of industrial and scientific applications.

\section{Bibliography}

[1] G. Elert, Width of a human hair, The Physics Factbook (1999).

[2] G. Stevens, Microphotography (J. Wiley \& Sons, 1968).

[3] J. T. Bruning, Optical lithography - thirty years and three orders of magnitude: the evolution of optical lithography tools, Proceedings of SPIE 3051, 14 (1997).

[4] T. Matsuyama, Y. Ohmura, and D. M. Williamson, The lithographic lens: its history and evolution, Proceedings of SPIE 6154, 615403 (2006).

[5] J. H. Bruning, Optical lithography: 40 years and holding, Proceedings of SPIE 6520, 652004 (2007).

[6] C. Wagner and N. Harned, EUV lithography: Lithography gets extreme, Nature Photonics 4, 24 (2010).

[7] G. Tallents, E. Wagenaars, and G. Pert, Optical lithography: Lithography at EUV wavelengths, Nature Photonics 4, 809 (2010).

[8] EUV lithography, edited by V. Bakshi (SPIE Press, Bellingham, Washington, 2008).

[9] I. A. Makhotkin, E. Zoethout, E. Louis, A. M. Yakunin, S. Müllender, and F. Bijkerk, Wavelength selection for multilayer coatings for lithography generation beyond extreme ultraviolet, Journal of Micro/Nanolithography MEMS and MOEMS 11, 040501 (2012). 
[10] I. A. Makhotkin, E. Zoethout, E. Louis, A. M. Yakunin, S. Müllender, and F. Bijkerk, Spectral properties of La/B - based multilayer mirrors near the boron K absorption edge, Optics Express 20, 11778 (2012).

[11] I. A. Makhotkin, E. Zoethout, R. W. E. van de Kruijs, L. E. Yakunin, S. N., A. M. Yakunin, S. Müllender, and F. Bijkerk, Short period La/B and LaN/B multilayer mirrors for 6.8 nm wavelength, Optics Express 20, 11778 (2012).

[12] D. Attwood, Soft x-rays and extreme ultraviolet radiation: principles and applications (Cambridge University Press, 2007).

[13] E. Louis, A. E. Yakshin, T. Tsarfati, and F. Bijkerk, Nanometer interface and materials control for multilayer EUV-optical applications, Progress in Surface Science 86, 255 (2011).

[14] V. Bakshi, EUV sources for lithography (SPIE press, 2006), Vol. 149.

[15] V. Banine, K. Koshelev, and G. Swinkels, Physical processes in EUV sources for microlithography, Journal of Physics D: Applied Physics 44, 253001 (2011).

[16] A. K. Ray-Chaudhuri, S. E. Gianoulakis, P. A. Spence, M. P. Kanouff, and C. D. Moen, Impact of thermal and structural effects on EUV lithographic performance, Proceedings of SPIE 3331, 124 (1998).

[17] Y. Li, K. Ota, and K. Murakami, Thermal and structural deformation and its impact on optical performance of projection optics for extreme ultraviolet lithography, Journal of Vacuum Science \& Technology B 21, 127 (2003).

[18] K. Liu, Y. Li, F. Zhang, and M. Fan, Transient thermal and structural deformation and its impact on optical performance of projection optics for extreme ultraviolet lithography, Japanese Journal of Applied Physics 46, 6568 (2007).

[19] G. Yang and Y. Li, Analysis and control of thermal and structural deformation of projection optics for 22-nm EUV lithography, Proceedings of SPIE 8322, 83222V (2012).

[20] R. Saathof, Adaptive optics to counteract thermal aberrations, Ph.D. thesis, Technische Universiteit Delft, The Netherlands, 2013.

[21] V. Belik, Y. M. Zadiranov, N. Il'inskaya, A. Korlyakov, V. Luchinin, M. Markosov, R. Seisyan, and E. Sher, Free-standing optical filters for a nanolithography source operating in the 12-15 $\mathrm{nm}$ wavelength range, Technical Physics Letters 33, 508 (2007).

[22] N. I. Chkhalo, M. N. Drozdov, E. B. Kluenkov, A. Y. Lopatin, V. I. Luchin, N. N. Salashchenko, N. N. Tsybin, L. A. Sjmaenok, V. E. Banine, and A. M. Yakunin, Free-standing spectral purity filters for extreme ultraviolet lithography, Journal of Micro/Nanolithography MEMS and MOEMS 11, 021115 (2012). 
[23] C. Mbanaso, A. Antohe, H. Bull, F. Goodwin, A. Hershcovitch, and G. Denbeaux, Out-of-band radiation mitigation at $10.6 \mu \mathrm{m}$ by molecular absorbers in laser-produced plasma extreme ultraviolet sources, Journal of Micro/Nanolithography MEMS and MOEMS 11, 021116 (2012).

[24] A. van den Boogaard, E. Louis, F. Van Goor, and F. Bijkerk, Optical element for full spectral purity from IR-generated EUV light sources, Proceedings of SPIE 72713B (2009).

[25] A. van den Boogaard, F. van Goor, E. Louis, and F. Bijkerk, Wavelength separation from extreme ultraviolet mirrors using phaseshift reflection, Optics Letters 37, 160 (2012).

[26] W. A. Soer, M. J. Jak, A. M. Yakunin, M. M. van Herpen, and V. Y. Banine, Grid spectral purity filters for suppression of infrared radiation in laser-produced plasma EUV sources, Proceedings of SPIE 72712Y (2009).

[27] W. Soer, P. Gawlitza, M. Van Herpen, M. Jak, S. Braun, P. Muys, and V. Banine, Extreme ultraviolet multilayer mirror with near-zero IR reflectance, Optics Letters 34, 3680 (2009).

[28] M. Van Herpen, R. van de Kruijs, D. Klunder, E. Louis, A. Yakshin, S. A. van der Westen, F. Bijkerk, and V. Banine, Spectral-purity-enhancing layer for multilayer mirrors, Optics Letters 33, 560 (2008).

[29] V. Medvedev, A. Yakshin, R. van de Kruijs, V. Krivtsun, A. Yakunin, K. Koshelev, and F. Bijkerk, Infrared suppression by hybrid EUV multilayer-IR etalon structures, Optics Letters 36, 3344 (2011).

[30] V. Medvedev, A. Yakshin, R. van de Kruijs, V. Krivtsun, A. Yakunin, K. Koshelev, and F. Bijkerk, Infrared antireflective filtering for extreme ultraviolet multilayer Bragg reflectors, Optics Letters 37, 1169 (2012).

[31] E. Hecht, Optics (Addison Wesley, San Francisco, 2002).

[32] P. Verardi, M. Dinescu, F. Craciun, R. Dinu, and M. F. Ciobanu, Growth of oriented $\mathrm{Pb}\left(Z r_{x} T i_{1-x}\right) \mathrm{O}_{3}$ thin films on glass substrates by pulsed laser deposition, Applied Physics A 69, S837 (1999).

[33] J. H. Kim and F. F. Lange, Epitaxial growth of $\mathrm{PbZr}_{0.5} \mathrm{Ti}_{0.5} \mathrm{O}_{3}$ thin films on (001) $\mathrm{LaAlO}_{3}$ by the chemical solution deposition method, Journal of Materials Research 14, 4004 (1999).

[34] M. Nakajima, S. Okamoto, H. Nakaki, T. Yamada, and H. Funakuboa, Enhancement of piezoelectric response in (100)/(001) oriented tetragonal $\mathrm{Pb}(\mathrm{Zr}, \mathrm{Ti}) \mathrm{O}_{3}$ films by controlling tetragonality and volume fraction of the (001) orientation, Journal of Applied Physics 109, 091601 (2011).

[35] J. Son and Y.-H. Shin, Highly c-Oriented $\mathrm{PbZr}_{0.48} \mathrm{Ti}_{0.52} \mathrm{O}_{3}$ Thin Films on Glass Substrates, Electrochemical and Solid-State Letters 12, G20 (2009). 
[36] D. H. Kim, Y. K. Kim, S. Hong, Y. Kim, and S. Baik, Nanoscale bit formation in highly (111)-oriented ferroelectric thin films deposited on glass substrates for high-density storage media, Nanotechnology 22, 245705 (2011).

[37] S. S. Roy, H. Gleeson, C. P. Shaw, R. W. Whatmore, Z. Huang, Q. Zhang, and S. Dunn, Growth and characterisation of lead zirconate titanate (30/70) on indium tin oxide coated glass for oxide ferroelectric-liquid crystal display application, Integrated Ferroelectrics 29, 189 (2000).

[38] K. K. Uprety, L. E. Ocola, and O. Auciello, Growth and characterization of transparent $\mathrm{Pb}(\mathrm{Zi}, \mathrm{Ti}) \mathrm{O}_{3}$ capacitor on glass substrate, Journal of Applied Physics 102, 084107 (2007).

[39] E. Bruno, F. Ciuchi, M. Castriota, S. Marino, G. Nicastro, E. Cazzanelli, and N. Scaramuzza, Structural transformations of PZT 53/47 sol-gel films on different substrates driven by thermal treatments, Ferroelectrics 396, 49 (2010).

[40] K. Lefki and G. J. M. Dormans, Measurement of piezoelectric coefficients of ferroelectric thin films, Journal of Applied Physics 76, 1764 (1994).

[41] J. W. Hardy, Adaptive optics for astronomical telescopes (Oxford University Press, New York, 1998).

[42] F. Roddier, Adaptive optics in astronomy (Cambridge University Press, Cambridge, 1999).

[43] Adaptive optics for vision science: Principles, practices, design and applications, edited by J. Porter, H. M. Queener, J. E. Lin, K. Thorn, and A. A. S. Awwal (John Wiley \& Sons, Inc, Hoboken, NJ, 2006).

[44] R. K. Tyson, Principles of adaptive optics, 3rd ed. (Taylor \& Francis Group, LLC, Boca Raton, Florida, 2011).

[45] Boston Micromachines Corporation, 2014.

[46] Iris AO, Inc., 2014.

[47] ALPAO SAS, 2014.

[48] Flexible Optical B.V. (Okotech), 2014.

[49] CILAS Corporate, 2014.

[50] AOA Xinetics, 2014.

[51] Microgate engineering, 2014.

[52] R. Hamelinck, R. Ellenbroek, N. Rosielle, M. Steinbuch, M. Verhaegen, and N. Doelman, Validation of a new adaptive deformable mirror concept, Proceedings of SPIE 7015, 70150Q (2008). 
[53] R. Hamelinck, Adaptive deformable mirror: based on electromagnetic actuators, Ph.D. thesis, Technische Universiteit Eindhoven, The Netherlands, 2010.

[54] P. Doel, C. Atkins, S. Thompson, D. Brooks, J. Yao, C. Feldman, R. Willingale, T. Button, D. Zhang, and A. James, Large thin adaptive x-ray mirrors, Proceedings of SPIE 6705, 67050M (2007).

[55] P. B. Reid, S. S. Murray, S. Trolier-McKinstry, M. Freeman, M. Juda, W. Podgorski, B. Ramsey, and D. Schwartz, Development of adjustable grazing incidence optics for Generation-x, Proceedings of SPIE 7011, 70110V (2008).

[56] W. N. Davis, P. B. Reid, and D. A. Schwartz, Finite element analyses of thin film active grazing incidence $x$-ray optics, Proceedings of SPIE 7803, 78030P (2010).

[57] D. Zhang, D. Rodriguez-Sanmartin, T. W. Button, C. Atkins, D. Brooks, P. Doel, C. Dunare, C. Feldman, A. James, A. Michette, W. Parkes, S. Pfauntsch, S. Sahraei, T. Stevenson, H. Wang, and R. Willingale, Development of piezoelectric actuators for active $x$-ray optics, Journal of Electroceramics 27, 1 (2011).

[58] V. Cotroneo, W. N. Davis, V. Marquez, P. B. Reid, D. A. Schwartz, R. L. Johnson-Wilke, S. E. Trolier-McKinstry, and R. H. T. Wilke, Adjustable grazing incidence $x$-ray optics based on thin PZT films, Proceedings of SPIE 8503, 850309 (2012).

[59] R. H. T. Wilke, R. L. Johnson-Wilke, V. Cotroneo, W. N. Davis, P. B. Reid, D. A. Schwartz, and S. Trolier-McKinstry, Sputter deposition of PZT piezoelectric films on thin glass substrates for adjustable $x$-ray optics, Applied Optics 52, 3412 (2013).

[60] H. Mimura, S. Handa, T. Kimura, H. Yumoto, D. Yamakawa, H. Yokoyama, S. Matsuyama, K. Inagaki, K. Yamamura, Y. Sano, K. Tamasaku, M. Nishino, Y. Yabashi, T. Ishikawa, and K. Yamauchi, Breaking the $10 \mathrm{~nm}$ barrier in hard-x-ray focusing, Nature Physics 6, 122 (2010).

[61] S. Matsuyama, T. Kimura, H. Nakamori, S. Imai, Y. Sano, Y. Kohmura, K. Tamasaku, M. Yabashi, T. Ishikawa, and K. Yamauchi, Development of piezoelectric adaptive mirror for hard $x$-ray nanofocusing, Proceedings of SPIE 8503, 850303 (2012).

[62] S. Ravensbergen, Adaptive optics for extreme ultraviolet lithography: Actuator design and validation for deformable mirror concepts, Ph.D. thesis, Technische Universiteit Eindhoven, The Netherlands, 2012.

[63] S. Ravensbergen, P. Rosielle, and M. Steinbuch, Deformable mirrors with thermo-mechanical actuators for extreme ultraviolet lithography: Design, realization and validation, Precision Engineering 37, 353 (2013). 
[64] S. Trolier-McKinstry and P. Muralt, Thin film piezoelectrics for MEMS, Journal of Electroceramics 12, 7 (2004).

[65] A. Pramanick, A. D. Prewitt, J. S. Forrester, and J. L. Jones, Domains, domain walls and defects in perovskite ferroelectric oxides: A review of present understanding and recent contributions, Critical Reviews in Solid State and Materials Sciences 37, 243 (2012).

[66] X.-H. Xu, B.-Q. Li, Y. Feng, and J.-R. Chu, Design, fabrication and characterization of a bulk-PZT-actuated MEMS deformable mirror, Journal of Micromechanics and Microengineering 17, 2439 (2007).

[67] Y. Hishinuma and E.-H. E. Yang, Piezoelectric unimorph micro actuator arrays for single-crystal silicon continuous-membrane deformable mirror, Journal of Microelectromechanical Systems 15, 370 (2006).

[68] R. Perez, J. Agnus, C. Clevy, A. Hubert, and N. Chaillet, Modeling fabrication and validation of a high-performance 2-DoF piezoactuator for micromanipulation, IEEE/ASME Transactions on Mechatronics 10, 161 (2005).

[69] D. D. Fong, G. B. Stephenson, S. K. Streiffer, J. A. Eastman, O. Auciello, P. H. Fuoss, and C. Thompson, Ferroelectricity in ultrathin perovskite films, Science 304, 1650 (2004).

[70] S. Graulis, A. Dakevi, A. Merkys, D. Chateigner, L. Lutterotti, M. Quirs, N. R. Serebryanaya, P. Moeck, R. T. Downs, and A. Le Bail, Crystallography Open Database (COD): an open-access collection of crystal structures and platform for world-wide collaboration, Nucleic Acids Research 40, D420 (2012).

[71] S. Grazulis, D. Chateigner, R. T. Downs, A. T. Yokochi, M. Quiros, L. Lutterotti, E. Manakova, J. Butkus, P. Moeck, and A. Le Bail, Crystallography Open Database - an open-access collection of crystal structures, Journal of Applied Crystallography 42, 726 (2009).

[72] R. T. Downs and M. Hall-Wallace, The american mineralogist crystal structure database, American Mineralogist 88, 247 (2003).

[73] R. E. Leuchtner and K. S. Grabowski, in Pulsed laser deposition of thin films, edited by D. B. Chrisey and G. K. Hubler (John Wiley \& Sons, Inc., New York, 1994), Chap. Ferroelectrics. 


\section{Spectral purification and infrared light recycling in extreme ultraviolet lithography sources}

We present the design of a novel collector mirror for laser produced plasma (LPP) light sources to be used in extreme ultraviolet (EUV) lithography. The design prevents undesired infrared (IR) drive laser light, reflected from the plasma, from reaching the exit of the light source. This results in a strong purification of the EUV light, while the reflected IR light becomes refocused into the plasma for enhancing the IR-to-EUV conversion. The dual advantage of EUV purification and conversion enhancement is achieved by incorporating an IR Fresnel zone plate pattern into the EUV reflective multilayer coating of the collector mirror. Calculations using Fresnel-Kirchhoff's diffraction theory for a typical collector design show that the IR light at the EUV exit is suppressed by four orders of magnitude. Simultaneously, $37 \%$ of the reflected IR light is refocused back the plasma. 


\subsection{Introduction}

Electronic devices are becoming more powerful and more energy efficient, the key to this progress being the ongoing miniaturization in the semiconductor industry. Integrated circuits (IC) fabricated with current lithography, using ultraviolet (UV) light at $193 \mathrm{~nm}$ wavelength, can have feature sizes as small as $32 \mathrm{~nm}$ [1]. On the other hand, this lithography technique is close to the physical limit to fabricate even smaller IC features. This is why the semiconductor industry is aiming to introduce extreme ultraviolet (EUV) lithography at a wavelength of $13.5 \mathrm{~nm}$. Due to the extremely short wavelength it is possible to fabricate sub$22 \mathrm{~nm}$ features [1,2]. Consequently, the development of according EUV light sources with high power and clean, in-band radiation output $[3,4]$ is one of the major challenges.

The currently most promising approaches for generating EUV radiation in the named range are based on discharge produced plasmas $[1,3,4]$ and laser produced plasmas (LPP) $[1,3-5]$. The latter method is scalable to higher output powers and is therefore of interest for high volume production [6-9] in a lithographic tool. Figure 2.1(a) shows the standard schematic setup of an LPP source for generating EUV radiation at $13.5 \mathrm{~nm}$ wavelength with a hot tin ( $\mathrm{Sn}$ ) plasma. High-power, short laser pulses from an IR drive laser are focused to small tin droplets. The drive laser is typically a $\mathrm{CO}_{2}$ laser with an IR wavelength of $10.6 \mu \mathrm{m}$ and pulse duration around $100 \mathrm{~ns}$ [4]. The laser vaporizes and ionizes the tin to obtain a hot plasma which emits strong EUV radiation. In order to collect a maximum amount of the EUV radiation, a large-angle ellipsoidal mirror with a highly EUV reflective Mo/Si multilayer coating [3, 4] is employed. The curvature and positioning of this so-called collector mirror is chosen to have its first focus located in the plasma. This setting directs the EUV into the secondary focus of the ellipsoid. In the secondary focus an aperture matching the size of the EUV beam forms the exit of the source towards the lithography optical system.

This standard collector approach can collect substantial EUV powers [3, 4], however there are two clear disadvantages. These occur because the plasma reflects a considerable part of the incident drive laser. The first problem is that this reflected drive laser light is lost for the plasma heating process. The second problem is that the reflected drive laser radiation is directed into the most undesired location, the intermediate focus, from where it can exit together with EUV radiation. This is highly undesired because the high power of the IR radiation causes heating of the photoresist materials used in lithography process.

Potential approaches for suppressing the IR radiation have been suggested, such as membrane [10, 11] and gaseous filters [12]. However, these techniques may cause thermally induced optical distortions. Solutions based on interference techniques have been proposed as well [13-19] but these schemes do not recycle any reflected IR light. A recycling of the lost radiation has not been addressed so far.

Here we propose a new method that solves both problems simultaneously, via incorporating a Fresnel zone plate in the form of a diffractive pattern in the surface of the EUV reflective collector. The zone plate removes undesired IR 
light from the EUV exit of the source and diverts the removed IR light back into the plasma for further heating and increasing the IR-to-EUV conversion efficiency. In Fig. 2.1(b) we compare the IR and EUV beam paths obtained with the zone plate assisted collector to the standard situation shown in Fig. 2.1(a). It can be seen in Fig. 2.1(b) that the EUV radiation (shown in light blue) follows a completely different path than the IR light reflected by the plasma (shown as dark red stripes). Specifically, only the EUV radiation purified from the IR radiation leaves the exit while the reflected IR light is refocused into the plasma.

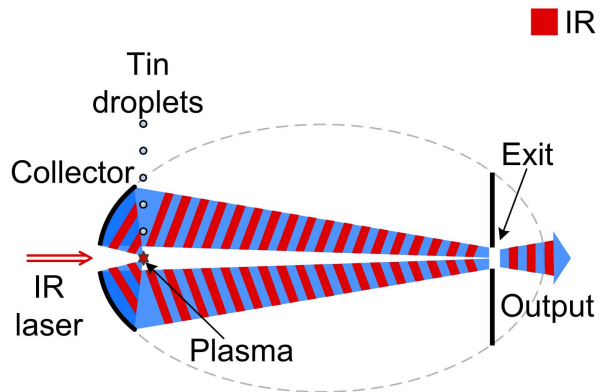

(a)

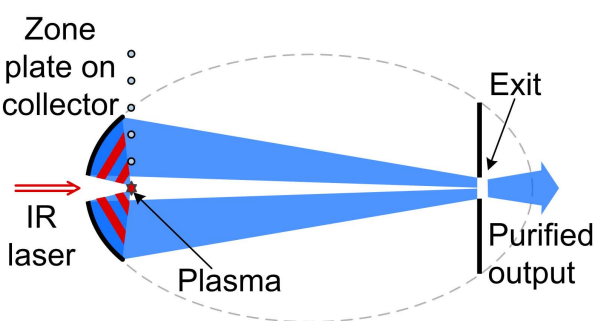

(b)

Figure 2.1: Schematic drawing of (a) a typical EUV source based on laser produce plasma (LPP). The reflected IR and generated EUV light both follow the same path through the exit (red-blue area). (b) The same source using a Fresnel zone plate on the collector mirror. Only the EUV passes through the exit aperture (blue), while reflected IR (red) is refocused into the plasma.

\subsection{Calculation of Fresnel zone pattern}

In this section we present the physical action of our approach in more detail. The goal is to calculate the dimensions of a Fresnel pattern as required for refocusing the IR light reflected from the plasma back into the plasma. For the calculations we refer to Fig. 2.2 which shows the cross-section of an ellipsoidal collector, the surface of which is carrying a binary Fresnel zone pattern. The pattern is made of Fresnel zones of width $w_{n}$, extending from a radius $r_{n-1}$ to $r_{n}\left(w_{n}=r_{n}-r_{n-1}\right)$. As we are considering a reflective Fresnel zone pattern, we chose the depth of the Fresnel zones as $h=\lambda / 4$ to maximize the interference contrast where $\lambda$ is the IR wavelength. The collector is positioned to overlap its first focus at point $F$ with the plasma. In ordinary collectors without a Fresnel pattern, all the light from the plasma (both the reflected IR and generated EUV) is directed by the ellipsoidal surface to the intermediate focus. In contrast, when the collector is structured with a Fresnel zone pattern, the reflected IR light can be refocused into the plasma. For the determination of the required Fresnel zone dimensions we approximate the drive laser light reflected from the plasma as a 
point source located at point $F$. In this case, refocusing of the radiation back to $F$ is achieved when there is constructive interference of light from all Fresnel zones [20]. In Fig. 2.2 this corresponds to constructive interference of the on-axis path $\overline{F A F}$ with the off-axis paths $\overline{F B F}$, which can be written as:

$$
2\left(\sqrt{d_{n}^{2}+r_{n}^{2}}\right)-2 d_{0}=n \lambda / 2
$$

where $d_{n}$ is the longitudinal and $r_{n}$ is the radial distance of point $B$ from point $F, d_{0}$ is the longitudinal distance between the center of the collector (point $A$ ) and the first focus (point $F$ ), $n=1,2,3, \ldots$ is an integer representing the numbering of the Fresnel zones. Knowing that point $A$ lies on the elliptic contour of the mirror, as taken in this example, the distance $d_{0}$ can be written as $d_{0}=a-\sqrt{a^{2}-b^{2}}$ where $a$ and $b$ are the major and minor axes of the elliptical mirror contour, respectively. Inserting $d_{0}$ into Eq. (2.1) yields:

$$
2\left(\sqrt{d_{n}^{2}+r_{n}^{2}}\right)-2\left(a-\sqrt{a^{2}-b^{2}}\right)=n \lambda / 2
$$

Also point $B$ lies on the elliptical contour, therefore $d_{n}$ and $r_{n}$ in the Eq. (2.2) are mutually related via the equation for an ellipse:

$$
\left(\frac{d_{n}+\sqrt{a^{2}-b^{2}}}{a}\right)^{2}+\left(\frac{r_{n}}{b}\right)^{2}=1
$$

Inserting Eq. (2.3) into Eq. (2.2) and solving for $r_{n}$ yields the widths and radii of the Fresnel zone pattern required for refocusing:

$$
w_{n}=r_{n}-r_{n-1} \text { with } r_{n}=b \sqrt{-\left(\frac{b^{2}+t^{2}-2 a t}{a^{2}-b^{2}}\right)}
$$

where $t=n \lambda / 4+a-\sqrt{a^{2}-b^{2}}$.

To this end we point to an important property of the Fresnel pattern determined by Eq. (2.4). If we insert for $\lambda$ the relatively long wavelength of the IR drive laser, typically $10.6 \mu \mathrm{m}$, the width of the Fresnel zones turn out to be several orders of magnitude larger than the Fresnel zone width required to focus the EUV light to the same point. As a result, diffractive focusing power of the Fresnel pattern for the EUV is several orders of magnitude weaker than for the IR. Therefore the change of the EUV spot size in the exit plane caused by the Fresnel pattern can be neglected compared to the original EUV spot size. We note that close to the edges of the Fresnel zones the multilayer period may deviate from its intended value, which would cause some EUV loss. However, it shows that the surface fraction of these edges and therefore also the according loss is very small, in the range of $1 \%$ [21]. Improved deposition techniques are available that further reduce this fraction [22]. 


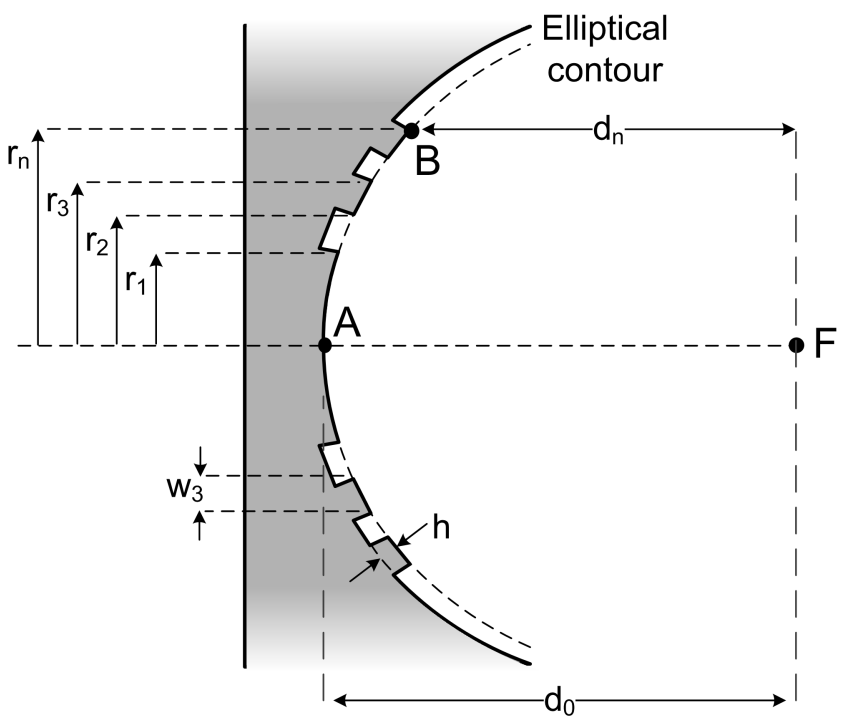

Figure 2.2: Fresnel zone pattern at the surface of an ellipsoidal collector with focus point $F$.

\subsection{Numerical results}

Now that the required dimensions of the Fresnel zones can be determined with Eq. (2.4), we turn to the quantification of purification and refocusing by calculating the transverse intensity distributions in the plasma plane and the exit plane. In order to obtain results that are relevant for applications, we do the calculations with typical parameters of current sources. Such sources often incorporate $\mathrm{CO}_{2}$ lasers at $10.6 \mu \mathrm{m}$ wavelength as drive lasers and a typical collector that have opening angles between $1.6 \mathrm{sr}$ and $5 \mathrm{sr}$, and with collector diameters between $300 \mathrm{~mm}$ and $100 \mathrm{~mm}$, respectively [23]. These numbers correspond to major and minor axes lengths, $a$ and $b$, of $1000 \mathrm{~mm}$ and $600 \mathrm{~mm}$, respectively. From these numbers, the collector distance $d_{0}$ in Fig. 2.2 can be calculated as $d_{0}=200 \mathrm{~mm}$.

In the next step, we use the Fresnel-Kirchhoff diffraction theory [20] to calculate the intensity in the plasma plane and the exit plane. We use the parameters as given above and apply the point source approximation again. The latter is justified if the diameter of the source (i.e. the plasma diameter) can be neglected with regard to the distance to the collector. The plasma diameter in current sources (full width at half maximum, FWHM) lies in the range of $100 \mu \mathrm{m}$ to $300 \mu \mathrm{m}[4-10]$ which can be safely neglected with respect to the collector distance.

The calculation of the intensity distribution using the Fresnel-Kirchhoff diffraction theory is a computationally rather involved procedure, therefore we restrict ourselves to a maximum number of 200 Fresnel zones which corresponds to maximum patterned area of $44 \mathrm{~mm}$ diameter. This is much smaller than the typical 
$300 \mathrm{~mm}$ to $600 \mathrm{~mm}$ diameter used in a real source setup. If we would calculate the Fresnel-Kirchhoff diffraction for a fully patterned typical collector, the purified and refocused power would be higher due to the larger area. Also the focusing diameter would be smaller which is a well-known property of Fresnel zone plates [20]. This means that our limit of $44 \mathrm{~mm}$ yields a conservative calculation of the purification and recycling factors.

In Fig. 2.3 we present the calculated diameter (FWHM) of the intensity distribution generated by the Fresnel zone patterned collector in the plane of the plasma. In the calculations we have considered that the collector mirror is equipped with an opening for letting the drive laser beam enter. For simplicity we choose an opening with a diameter of $1.5 \mathrm{~mm}$ which is equal to the diameter of the first Fresnel zone. It can be seen in Fig. 2.3 that, indeed, the diameter of the IR focus decreases steadily with an increasing number of Fresnel zones included in the calculations. This decrease is of interest because it indicates how many Fresnel zones are required in order to obtain a focus smaller than the plasma diameter such that essentially all refocused light becomes recycled to the plasma for additional heating. For example, the diameter of the Fresnel focus becomes smaller than the plasma diameters of $300 \mu \mathrm{m}$ and $100 \mu \mathrm{m}$ if more than approximately 10 and 130 Fresnel zones are used respectively. In the rest of the intensity calculations we restrict ourselves to 130 Fresnel zones which correspond to $35 \mathrm{~mm}$ collector diameter.

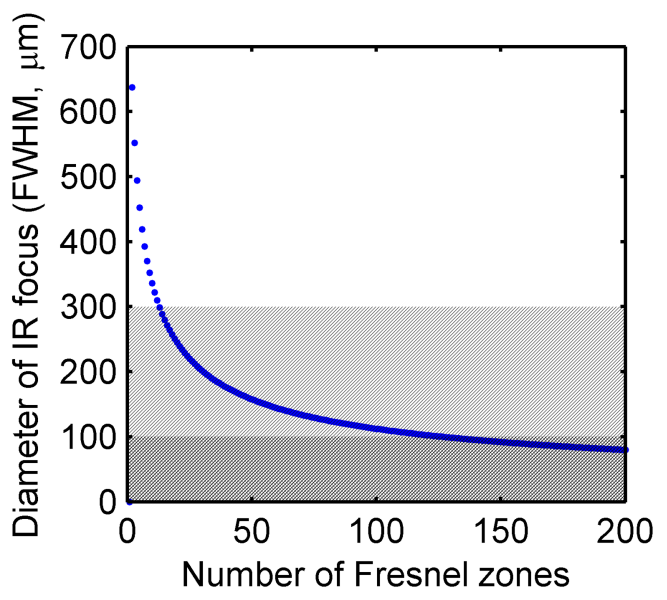

Figure 2.3: Decrease of the focus diameter (FWHM) generated by the Fresnel zone pattern in the plasma plane versus the number of contributing Fresnel zones. Currently used plasma sources have a diameter between $300 \mu \mathrm{m}$ and $100 \mu \mathrm{m}$ which is indicated by the light and dark shaded areas.

Figure 2.4 shows the calculated transverse IR intensity distribution in the plasma plane. The two upper graphs compare the intensity distributions generated with (a) the zone plate assisted collector to the intensity with (b) a standard ellipsoidal collector without a Fresnel zone pattern. The two lower graphs show 
the corresponding cross-sections of the intensity distributions on a logarithmic scale. Comparing the Fig. 2.4(a) and (b) it can be seen that the IR laser intensity at the plasma location is enhanced by four orders of magnitude when the collector is patterned with Fresnel zones. Of more relevance is the fraction of the reflected IR light that is refocused to the plasma. To determine this fraction we take the ratio of the total power contained in Fig. 2.4(b) to the total power contained in a circle matching the plasma size for the intensity distribution in Fig. 2.4(a). We find a recycling factor of $37 \%$ and $31 \%$ for plasma diameters of $300 \mu \mathrm{m}$ and $100 \mu \mathrm{m}$, respectively which compares well with the focusing efficiency of binary Fresnel zone plates. This suggests that the recycling factor can be further increased such as using a multilevel Fresnel zone plate [24].
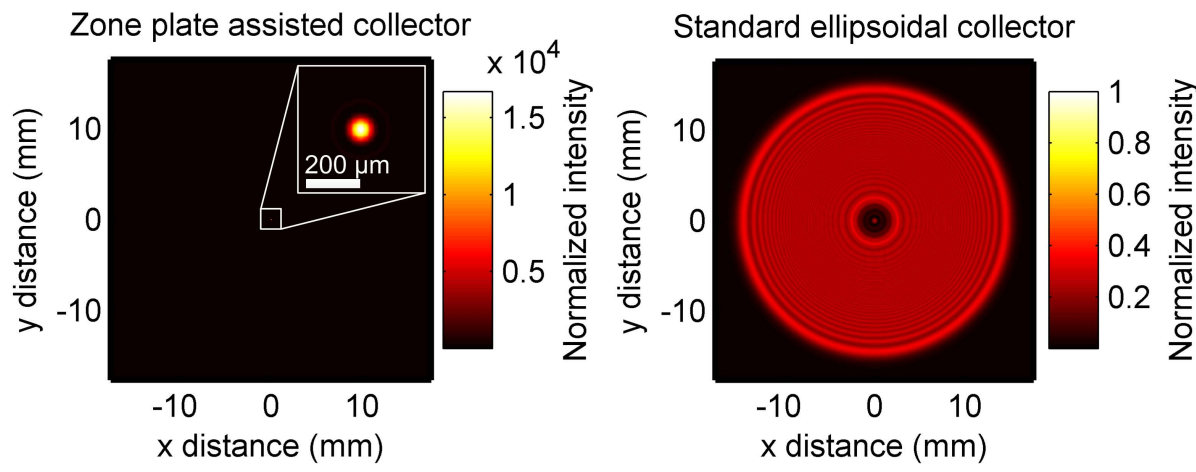

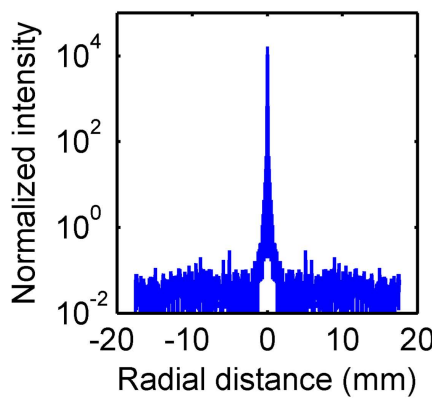

(a)

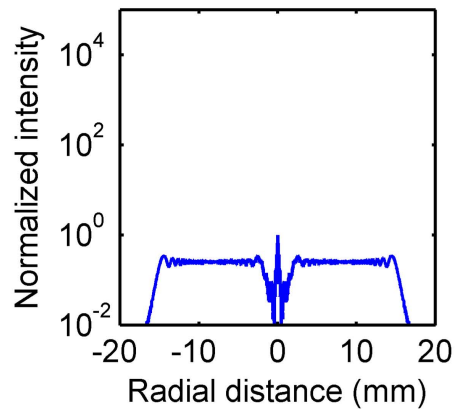

(b)

Figure 2.4: Intensity distribution at the first focal plane of the collector where plasma is positioned (a) with and (b) without the zone plate on the collector, zoomed version is shown in the inset.

In the second step, in order to determine the intensity distribution in the exit plane, we repeat the Fresnel-Kirchhoff calculation for that plane. The calculated distributions are shown in Fig. 2.5 where (a) corresponds to the zone plate assisted collector whereas (b) corresponds to the standard collector with cross-sections in logarithmic scale in the lower row. It can be seen that there is only very low IR 
intensity in the EUV exit plane. When looking at the centre of the distributions we find a four-orders-of-magnitude lower intensity.

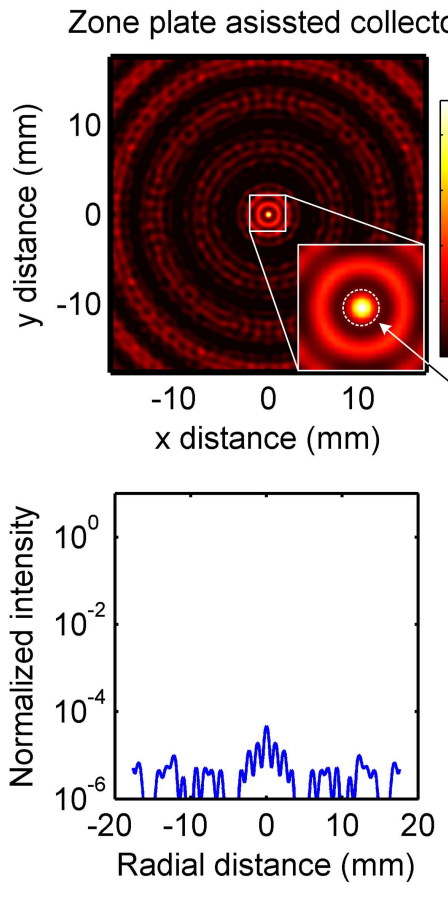

(a)

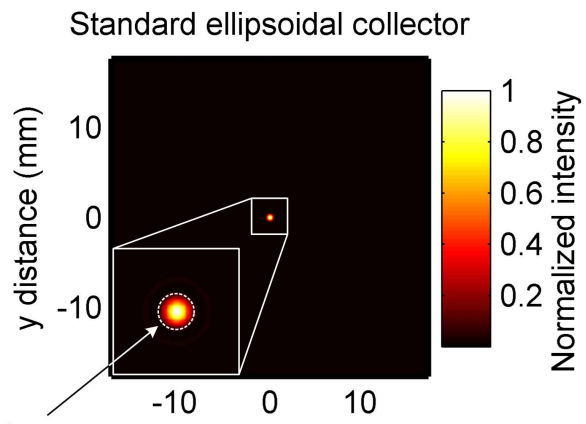

Exit aperture $\quad x$ distance $(\mathrm{mm})$ $(900 \mu \mathrm{m}$ diameter $)$

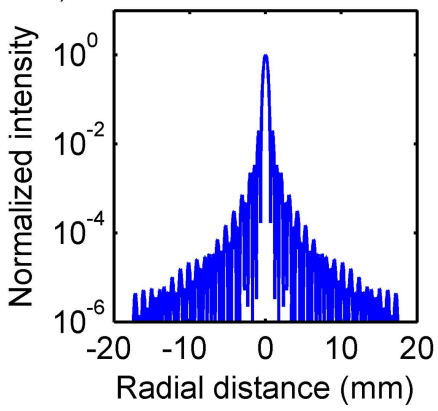

(b)

Figure 2.5: Intensity distribution at the intermediate focal plane of the collector which is also the exit of the source (a) with and (b) without the Fresnel zone plate on the collector. Exit apertures with $900 \mu \mathrm{m}$ diameter are shown in the zoomed insets.

In order to address to the intensity distribution a purification factor we select a relevant diameter for the exit aperture matching the image of the plasma. The diameter of the plasma image can be determined by the collector magnification, $M$, and the diameter of the plasma. The collector magnification is given by $M=\left(2 a-d_{0}\right) / d_{0}$. In our case we have $M=9$ which means that the diameter of the plasma image is between $900 \mu \mathrm{m}$ and $2700 \mu \mathrm{m}$. When an exit aperture of the image size is chosen we obtain an IR purification between $1.9 \times 10^{4}$ and $0.41 \times 10^{4}$ for plasma diameters between $100 \mu \mathrm{m}$ and $300 \mu \mathrm{m}$, respectively.

\subsection{Conclusion}

We present a novel collector design for EUV light sources using a laser produced plasma. The novel collector is based on a binary Fresnel zone pattern incorporated into the surface of a standard ellipsoidal collector. The design prevents 
undesired IR drive laser light, reflected from the plasma, from reaching to the exit of the light source. This results in a strong purification of the usable EUV light. The purification factor achieved is four orders of magnitude in the presented example. The fraction of the reflected IR light that is refocused to the plasma is $37 \%$. This recycling factor might be further increased with multilevel Fresnel zone patterns, for example, above $95 \%$ with 16 levels [24]. This potential is highly attractive for recycling a substantial amount of the reflected IR drive laser light. This novel approach can also be used in other applications where selective suppression and refocusing of the light at two largely different wavelengths is required such as in pump-probe or Raman measurements.

\section{Acknowledgements}

This research program is cofinanced by Stichting Technologie en Wetenschap (STW) under the project "Smart Multilayer Interactive Optics for Lithography at Extreme UV wavelengths (SMILE)" and Stichting voor Fundamenteel Onderzoek der Materie (FOM) under the programme "Controlling photon and plasma induced processes at EUV optical surfaces (CP3E)". The CP3E programme is cofinanced by Nederlandse Organisatie voor Wetenschappelijk Onderzoek (NWO), Carl Zeiss SMT GmbH (Oberkochen), ASML (Veldhoven), and the AgentschapNL through the Catrene EXEPT program.

\section{Bibliography}

[1] C. Wagner and N. Harned, EUV lithography: Lithography gets extreme, Nature Photonics 4, 24 (2010).

[2] G. Tallents, E. Wagenaars, and G. Pert, Optical lithography: Lithography at EUV wavelengths, Nature Photonics 4, 809 (2010).

[3] V. Bakshi, EUV sources for lithography (SPIE press, 2006), Vol. 149.

[4] V. Banine, K. Koshelev, and G. Swinkels, Physical processes in EUV sources for microlithography, Journal of Physics D: Applied Physics 44, 253001 (2011).

[5] T. Tomie, Tin laser-produced plasma as the light source for extreme ultraviolet lithography high-volume manufacturing: history, ideal plasma, present status, and prospects, Journal of Micro/Nanolithography MEMS and MOEMS 11, 021109 (2012).

[6] G. O'Sullivan and B. Li, Development of laser-produced plasma sources for extreme ultraviolet lithography, Journal of Micro/Nanolithography MEMS and MOEMS 11, 021108 (2012).

[7] I. V. Fomenkov, B. La Fontaine, D. Brown, I. Ahmad, P. Baumgart, N. R. Böwering, D. C. Brandt, A. N. Bykanov, S. De Dea, A. I. Ershov, et al., 
Development of stable extreme-ultraviolet sources for use in lithography exposure systems, Journal of Micro/Nanolithography MEMS and MOEMS 11, 021110 (2012).

[8] J. Fujimoto, T. Abe, S. Tanaka, T. Ohta, T. Hori, T. Yanagida, H. Nakarai, and H. Mizoguchi, Laser-produced plasma-based extreme-ultraviolet light source technology for high-volume manufacturing extreme-ultraviolet lithography, Journal of Micro/Nanolithography MEMS and MOEMS 11, 021111 (2012).

[9] R. S. Abhari, B. Rollinger, A. Z. Giovannini, O. Morris, I. Henderson, and S. S. Ellwi, Laser-produced plasma light source for extreme-ultraviolet lithography applications, Journal of Micro/Nanolithography MEMS and MOEMS 11, 021114 (2012).

[10] V. Belik, Y. M. Zadiranov, N. Il'inskaya, A. Korlyakov, V. Luchinin, M. Markosov, R. Seisyan, and E. Sher, Free-standing optical filters for a nanolithography source operating in the 12-15 $\mathrm{nm}$ wavelength range, Technical Physics Letters 33, 508 (2007).

[11] N. I. Chkhalo, M. N. Drozdov, E. B. Kluenkov, A. Y. Lopatin, V. I. Luchin, N. N. Salashchenko, N. N. Tsybin, L. A. Sjmaenok, V. E. Banine, and A. M. Yakunin, Free-standing spectral purity filters for extreme ultraviolet lithography, Journal of Micro/Nanolithography MEMS and MOEMS 11, 021115 (2012).

[12] C. Mbanaso, A. Antohe, H. Bull, F. Goodwin, A. Hershcovitch, and G. Denbeaux, Out-of-band radiation mitigation at $10.6 \mu \mathrm{m}$ by molecular absorbers in laser-produced plasma extreme ultraviolet sources, Journal of Micro/Nanolithography MEMS and MOEMS 11, 021116 (2012).

[13] A. van den Boogaard, E. Louis, F. Van Goor, and F. Bijkerk, Optical element for full spectral purity from IR-generated EUV light sources, Proceedings of SPIE 72713B (2009).

[14] A. van den Boogaard, F. van Goor, E. Louis, and F. Bijkerk, Wavelength separation from extreme ultraviolet mirrors using phaseshift reflection, $\mathrm{Op}$ tics Letters 37, 160 (2012).

[15] W. A. Soer, M. J. Jak, A. M. Yakunin, M. M. van Herpen, and V. Y. Banine, Grid spectral purity filters for suppression of infrared radiation in laser-produced plasma EUV sources, Proceedings of SPIE 72712Y (2009).

[16] W. Soer, P. Gawlitza, M. Van Herpen, M. Jak, S. Braun, P. Muys, and V. Banine, Extreme ultraviolet multilayer mirror with near-zero IR reflectance, Optics Letters 34, 3680 (2009).

[17] M. Van Herpen, R. van de Kruijs, D. Klunder, E. Louis, A. Yakshin, S. A. van der Westen, F. Bijkerk, and V. Banine, Spectral-purity-enhancing layer for multilayer mirrors, Optics Letters 33, 560 (2008). 
[18] V. Medvedev, A. Yakshin, R. van de Kruijs, V. Krivtsun, A. Yakunin, K. Koshelev, and F. Bijkerk, Infrared suppression by hybrid EUV multilayer-IR etalon structures, Optics Letters 36, 3344 (2011).

[19] V. Medvedev, A. Yakshin, R. van de Kruijs, V. Krivtsun, A. Yakunin, K. Koshelev, and F. Bijkerk, Infrared antireflective filtering for extreme ultraviolet multilayer Bragg reflectors, Optics Letters 37, 1169 (2012).

[20] E. Hecht, Optics (Addison Wesley, San Francisco, 2002).

[21] A. van den Boogaard, E. Louis, E. Zoethout, K. Goldberg, and F. Bijkerk, Characterization of Mo/Si multilayer growth on stepped topographies, Journal of Vacuum Science \& Technology B 29, 051803 (2011).

[22] D. Voronov, P. Gawlitza, R. Cambie, S. Dhuey, E. Gullikson, T. Warwick, S. Braun, V. Yashchuk, and H. Padmore, Conformal growth of Mo/Si multilayers on grating substrates using collimated ion beam sputtering, Journal of Applied Physics 111, 093521 (2012).

[23] D. C. Brandt, I. V. Fomenkov, A. I. Ershov, W. N. Partlo, D. W. Myers, N. R. Böwering, A. N. Bykanov, G. O. Vaschenko, V. Khodykin, J. R. Hoffman, et al., LPP EUV source development for HVM, Proceedings of SPIE 65170Q (2007).

[24] J. Jahns and S. J. Walker, Two-dimensional array of diffractive microlenses fabricated by thin film deposition, Applied Optics 29, 931 (1990). 



\section{Nanosheet controlled epitaxial growth of $\mathrm{PbZr}_{0.52} \mathrm{Ti}_{0.48} \mathrm{O}_{3}$ thin films on glass substrates}

Integration of $\mathrm{PbZr}_{0.52} \mathrm{Ti}_{0.48} \mathrm{O}_{3}$ (PZT) films on glass substrates is of high importance for device applications. However, to make use of the superior ferro- and piezoelectric properties of PZT, well-oriented crystalline or epitaxial growth with control of the crystal orientation is a prerequisite. In this article, we report on epitaxial growth of PZT films with (100)- and (110)-orientation achieved by utilizing $\mathrm{Ca}_{2} \mathrm{Nb}_{3} \mathrm{O}_{10}$ (CNO) and $\mathrm{Ti}_{0.87} \mathrm{O}_{2}$ (TO) nanosheets as crystalline buffer layers. Fatigue measurements demonstrated stable ferroelectric properties of these films up to $5 \times 10^{9}$ cycles. (100)-oriented PZT films on CNO nanosheets show a large remnant polarization of $21 \mu \mathrm{C} / \mathrm{cm}^{2}$ that is the highest remnant polarization value compared to (110)-oriented and polycrystalline films reported in this work. A piezoelectric response of $98 \mathrm{pm} / \mathrm{V}$ is observed for (100)-oriented PZT film which is higher than the values reported in the literature on Si substrates. 


\subsection{Introduction}

In the last few decades lead zirconate titanate, $\mathrm{PbZr}_{x} \mathrm{Ti}_{1-x} \mathrm{O}_{3}(\mathrm{PZT})$, thin films have found a plethora of applications in microelectromechanical systems (MEMS) [1] and memory devices [2]. The choice of PZT in these applications stems from its remarkable ferroelectric and piezoelectric properties [3]. These properties are strongly related to the quality of the crystal growth and the orientation of the PZT thin films $[4,5]$, therefore well-oriented crystalline growth or epitaxial growth with control of the orientation is highly desired. In addition, stability of the ferroelectric response after many switching cycles is a vital factor for longterm operation of the fabricated devices. $\mathrm{SrTiO}_{3}$ (STO) $[6,7], \mathrm{MgO}[8]$ and $\mathrm{LaAlO}_{3}$ (LAO) [9] substrates facilitate the epitaxial growth of PZT films due to three factors. (a) These substrates are stable against oxidation in the substrate thin film interface, (b) they are single crystalline and (c) the mismatch in the in-plane lattice parameters between the substrate and the film is small. On the other hand these substrates are difficult to process and expensive, therefore they have limited use in practical device applications. For practical applications, integration of PZT films on widely used inexpensive substrates such as $\mathrm{Si}$ and glass is required. Integration on glass is of high importance in order to pave the way to integrate $\mathrm{PZT}$ on amorphous structures, such as $\mathrm{SiO}_{2}$ that is commonly used in semiconductor devices and Mo/Si mirror coatings for extreme ultraviolet (EUV) photolithography $[10,11]$.

Deposition of oxide films, including PZT, directly on Si substrates results in inter-diffusion of oxides into $\mathrm{Si}$ and formation of amorphous oxide layer in the substrate-film interface, therefore buffer layers are utilized to prevent the inter-diffusion process. STO and yttria-stabilized zirconia (YSZ)/ $\mathrm{CeO}_{2}$ are such buffer layers that are commonly used to integrate epitaxial PZT films on Si substrates [12-15]. However growth of these buffer layers require high temperatures, therefore they are not suitable for many applications. Integration of PZT films on Si using Pt buffer layers (also serves as bottom electrode) is a low temperature process, but the films show poor ferroelectric properties $[16,17]$. Recently, integration of PZT films on Si have been achieved using nanometer-thick crystalline layers, namely nanosheets [18]. Since the integration using nanosheets was achieved at $600{ }^{\circ} \mathrm{C}$, this gives an opportunity to extend the integration of PZT on glass using nanosheets.

Initial growth studies on glass for flat panel display applications suffer from both mixed growth (pyrochlore and perovskite phase) and random orientation of the PZT films [19-24]. It is well known that the electrode material has a large impact on the growth quality, crystalline orientation and ferroelectric properties [14, 25-28]. Depositions on Pt or Au electrodes, that are known to have preferential growth at low temperatures, minimized the pyrochlore phase, but the resulting PZT films are polycrystalline [29-32]. Using conductive oxide electrodes such as $\mathrm{SrRuO}_{3}(\mathrm{SRO})$ and $\mathrm{LaNiO}_{3}$ (LNO), the orientation control has been improved and undesired crystal orientations have been reduced [33-36]. On the other hand, in these studies there is no detailed investigation of the piezoelectric properties, which is vital for device applications. 
In this paper we report on the growth of epitaxial (100)-oriented and preferentially (110)-oriented PZT films with LNO electrodes on glass substrates using $\mathrm{Ca}_{2} \mathrm{Nb}_{3} \mathrm{O}_{10}(\mathrm{CNO})$ and $\mathrm{Ti}_{0.87} \mathrm{O}_{2}$ (TO) nanosheet buffer layers, respectively. For comparison, a PZT film on Pt coated glass substrate was deposited under same growth conditions. In comparison to the PZT film deposited on Pt coated substrates, improved ferroelectric and piezoelectric properties were observed for the PZT films deposited on nanosheet buffered substrates. The whole PZT integration process including top and bottom electrode was achieved at growth temperature of $600{ }^{\circ} \mathrm{C}$ which makes it possible to extend the PZT deposition on amorphous structures with low processing temperatures.

\subsection{Experimental details}

The processes of nanosheets synthesis and transfer to the substrates have been done as described by Nijland et al. [37]. The CNO and TO nanosheets were obtained from layered compounds of $\mathrm{KCa}_{2} \mathrm{Nb}_{3} \mathrm{O}_{10}$ and $\mathrm{K}_{0.8}\left[\mathrm{Ti}_{1.73} \mathrm{Li}_{0.27}\right] \mathrm{O}_{4}$, respectively. Both layered compounds were treated by nitric acid in order to exchange potassium ions in the interlayers with protons resulting in $\mathrm{HCa}_{2} \mathrm{Nb}_{3} \mathrm{O}_{10} \cdot 1.5 \mathrm{H}_{2} \mathrm{O}$ and $\mathrm{H}_{1.7} \mathrm{Ti}_{1.73} \mathrm{O}_{4} \cdot \mathrm{H}_{2} \mathrm{O}$ solutions. The protonated compounds were treated by tetrabutylammonium to exfoliate the $\mathrm{CNO}$ and TO nanosheets. The nanosheets were transferred to the glass substrates using Langmuir-Blodgett deposition process, resulting in a dense coverage of the substrate surface. The substrates were ultra-low expansion (ULE) glass (Corning 7972) with $500 \mu \mathrm{m}$ thickness. Prior to the deposition, the nanosheet coated substrates were annealed at $600{ }^{\circ} \mathrm{C}$ for 60 minutes in 0.140 mbar oxygen pressure. This step helps to burn out any surfactant and increases the adhesion to the substrate. No peeling off was observed in the adhesion tests done with the scotch tape method. For the Pt sample, the glass substrate was first coated with $5 \mathrm{~nm}$ thick Ti to increase the adhesion and then with $100 \mathrm{~nm}$ thick $\mathrm{Pt}$ using radio frequency sputter deposition at room temperature.

It is well known that the ferroelectric and piezoelectric properties of the PZT films vary with the composition and the $\mathrm{PbZr}_{0.52} \mathrm{Ti}_{0.48} \mathrm{O}_{3}$ composition at the morphotropic phase boundary (MPB) is usually preferred due to its high piezoelectric response. In this article, $\mathrm{PbZr}_{0.52} \mathrm{Ti}_{0.48} \mathrm{O}_{3}$ films were grown using pulsed laser deposition (PLD) with a KrF excimer laser at $248 \mathrm{~nm}$ wavelength with $20 \mathrm{~ns}$ pulse duration. The PZT films were sandwiched between LNO electrodes. The base pressure of the deposition chamber was kept at $5 \times 10^{-7}$ mbar before increasing the temperature. The LNO and PZT layers were deposited at $600{ }^{\circ} \mathrm{C}$ and $585^{\circ} \mathrm{C}$, respectively. Crystallographic properties were determined by a Philips X'Pert MRD x-ray diffractometer (XRD) with $\mathrm{Cu}-\mathrm{K} \alpha$ radiation at $0.1548 \mathrm{~nm}$ wavelength. The cross-sectional scanning electron microscopy (SEM) images of the heterostructures were recorded using a Zeiss MERLIN HR-SEM. In order to enhance the homogeneity of the electric field, a $100 \mathrm{~nm}$ thick Pt film was deposited to every sample on the LNO top electrode using radio frequency sputtering at room temperature. Capacitors of $200 \times 200 \mu \mathrm{m}^{2}$ area were patterned using pho- 
tolithography and structured using standard Argon ion etching. Current-electric field $(I-E)$, polarization-electric field $(P-E)$ loops and the remnant polarization $\left(P_{r}\right)$ versus number of switching cycles (fatigue) were measured using an AixACCT TF Analyzer 2000 with bipolar triangular pulses at $1 \mathrm{kHz}$. The piezoelectric responses of the films were measured using a Polytec MSA-400 laser Doppler vibrometer (LDV) operating at $8 \mathrm{kHz}$.

\subsection{Results and discussion}

The measured XRD patterns of the heterostructures are shown in Fig. 3.1. The crystallographic peaks are labeled with pseudo-cubic indexing [38]. The peaks indicate a pure perovskite phase for all three heterostructures and no pyrochlore phase is observed. The heterostructures on CNO, TO and Pt yields pure (100)oriented, preferentially (110)-oriented and polycrystalline films, respectively. The pure (100)-oriented growth on CNO nanosheet can be attributed to the perfect match of the in-plane square lattices $(a=3.86 \AA)$ of the CNO and the LNO. This match prevents in-plane stress while the LNO film grows in (100) direction. Here it is important to note that each nanosheet flake has a regular square lattice matching the lattice of the LNO film therefore the growth is epitaxial in each flake. On the other hand distinct flakes can be rotated with respect to each other, therefore in-plane coherence across distinct flakes is not expected. The TO nanosheets have a $2 \mathrm{D}$ structure that is formed by edge linking of $\mathrm{TiO}_{6}$ octahedra in a lepidocrocite-type rectangular $2 \mathrm{D}$ lattice with in-plane lattice parameters of $a=3.76 \AA$ and $b=2.97 \AA$. The (100) face of the LNO cubic lattice $(a=3.86 \AA)$ has a large mismatch in $b$-parameter contrary to the close match in $a$-parameter. On the other hand, the (110) face of the LNO lattice $(a=3.86 \AA, b=5.45 \AA)$ forms a close match to the two-fold lattice of the TO nanosheet $(a=3.76 \AA$, $2 * b=5.94 \AA$ ). The resulting lattice mismatch, which is below $8 \%$, is small enough to allow preferential growth in (110) direction, but it is too large to achieve pure (110) growth, as evident from the small (111) peak visible in Fig. 3.1. The films on Pt layer shows mixture of distinct peaks with a strong (111) Pt peak as expected.

The microstructure of the deposited films is shown in Fig. 3.2, as analyzed using HR-SEM. The film stacks Pt/LNO/PZT/LNO, grown on CNO, TO and Pt layers are shown in (a), (b) and (c) respectively. The thicknesses of the bottom LNO, PZT and top LNO layers are approximately $200 \mathrm{~nm}, 750 \mathrm{~nm}$ and $200 \mathrm{~nm}$, respectively. The LNO film on CNO nanosheets has a clearly visible interface to the PZT layer and the densest packing as compared to the TO and Pt samples.

The measured polarization-electric field hysteresis, current-electric field switching loops and remnant polarization versus number of switching cycles are shown in Fig. 3.3(a), (b) and (c), respectively. The remnant polarization $\left(P_{r}\right)$ and coercive fields $\left(E_{c}\right)$ of the samples derived from Fig. 3.3(a) are listed in Table 3.1 in comparison to the values from literature. The remnant polarization for CNO, TO and Pt samples are $17 \mu \mathrm{C} / \mathrm{cm}^{2}, 8.3 \mu \mathrm{C} / \mathrm{cm}^{2}$ and $5.1 \mu \mathrm{C} / \mathrm{cm}^{2}$, respectively. The CNO sample has the highest and the $\mathrm{Pt}$ sample has the lowest remnant 


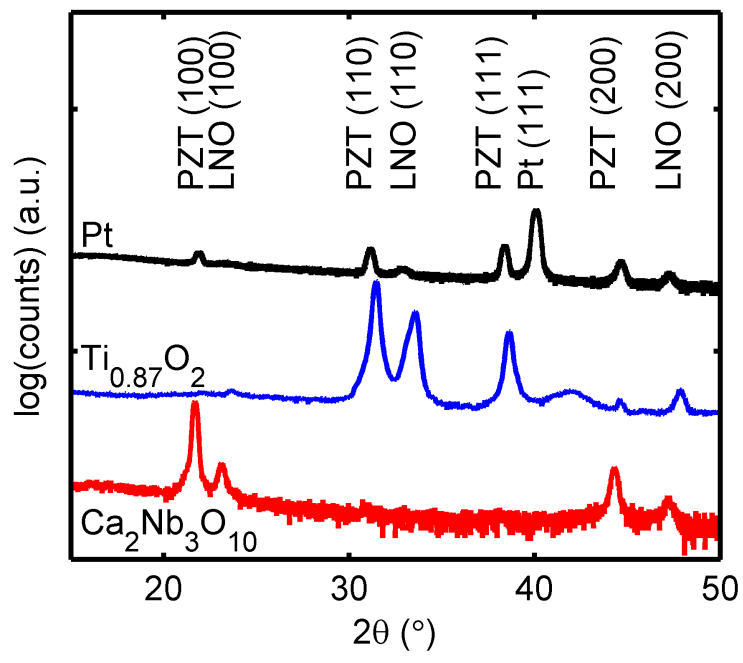

Figure 3.1: XRD $\theta-2 \theta$ scans of the $\mathrm{LaNiO}_{3} / \mathrm{PbZr}_{0.52} \mathrm{Ti}_{0.48} \mathrm{O}_{3} / \mathrm{LaNiO}_{3} /$ Buffer/Glass heterostructures deposited using $\mathrm{Pt}, \mathrm{Ti}_{0.87} \mathrm{O}_{2}$ and $\mathrm{Ca}_{2} \mathrm{Nb}_{3} \mathrm{O}_{10}$ buffer layers.

polarization, in relation to the epitaxial growth of the CNO sample and polycrystalline growth of the $\mathrm{Pt}$ sample. The remnant polarization and coercive field values from the literature show a large variation from $20 \mu \mathrm{C} / \mathrm{cm}^{2}$ to $32 \mu \mathrm{C} / \mathrm{cm}^{2}$ and from $33 \mathrm{kV} / \mathrm{cm}$ to $140 \mathrm{kV} / \mathrm{cm}$, respectively. On the other hand, the remnant polarization and coercive field values for our CNO sample are comparable to the values in Ref. [34] which has the same deposition temperature.
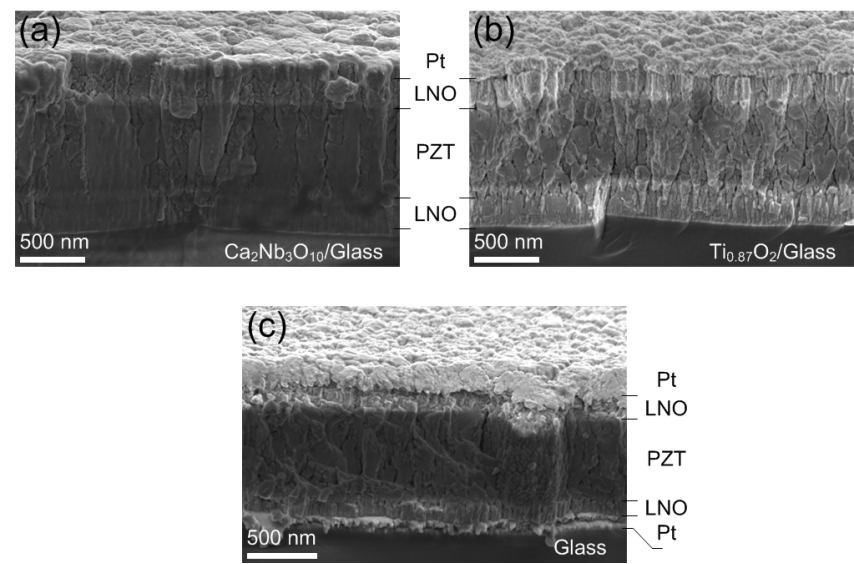

Figure 3.2: $\mathrm{SEM}$ images of the $\mathrm{PbZr}_{0.52} \mathrm{Ti}_{0.48} \mathrm{O}_{3}$-electrode stacks with (a) $\mathrm{Ca}_{2} \mathrm{Nb}_{3} \mathrm{O}_{10}$, (b) $\mathrm{Ti}_{0.87} \mathrm{O}_{2}$ and (c) $\mathrm{Pt}$ as buffer layers. 
The $I-E$ switching loops plotted in Fig. 3.3(b) shows a much sharper current peak for CNO sample than the other two peaks indicating the higher quality of the growth. The switching loop of the $\mathrm{Pt}$ sample has two peaks both in positive and negative switching directions. Existence of double switching peaks is well reported in the literature and is attributed to the pinning of the domain walls $[26,39]$. By subsequent cycling the domain walls are depinned leading to single switching peak. The remnant polarization of the films subjected to large number of switching cycles, namely the fatigue response, is shown in Fig. 3.3(c). After $5 \times 10^{9}$ switching cycles, the remnant polarization of the $\mathrm{CNO}$ and TO samples are constant as compared to the Pt sample. The remnant polarization of the Pt sample approximately doubles after the switching cycles are applied as a result of the depinning of the domain walls.

In the last step, the longitudinal piezoelectric responses $\left(d_{33, f}\right)$ of the PZT films were locally measured using LDV. The samples were fixed to large metal plates with silver paste to minimize the bending of the substrate (which would affect the measurement). Maximum piezoelectric response for CNO, TO and Pt are $98 \mathrm{pm}$, $49 \mathrm{pm}$ and $12 \mathrm{pm}$, respectively. The difference in the piezoelectric responses is a consequence of the difference in the orientations and the growth quality. Theoretically, highest piezoelectric response is predicted to be in (100)-oriented films and lowest in (111)-oriented films in accordance with our measurements [5]. The effective piezoelectric response depends not only on the orientation of the films but also on the ratio of the electrode size to the substrate thickness $[40,41]$. The CNO sample has higher piezoelectric response than the PZT films on Si substrates for similar electrode size to substrate thickness ratios [41]. Achieving this remarkable piezoelectric response on an amorphous substrate shows the effectiveness of the presented nanosheet approach in integrating ferroelectric PZT films on glass substrates.

\subsection{Conclusions}

In conclusion, we have grown and characterized epitaxial (100)-oriented and preferentially (110)-oriented $\mathrm{PbZr}_{0.52} \mathrm{Ti}_{0.48} \mathrm{O}_{3}$ films on glass substrates using $\mathrm{CNO}$ and TO nanosheets. The fabricated films show comparable or higher ferroelectric and piezoelectric responses than the values reported in the literature. The presented approach can also be used in integrating PZT films on amorphous surfaces for semiconductor circuits, glass waveguides and amorphous coatings like EUV mirrors.

\section{Acknowledgements}

Authors M. Bayraktar and A. Chopra both contributed equally to this article. This research program is funded by "Stichting Technologie en Wetenschap (STW)" under the contract 10448 with the project name "Smart Multilayer Interactive Optics for Lithography at Extreme UV wavelengths (SMILE)". The 
SMILE project is being sponsored by Carl Zeiss SMT GmbH. F. Bijkerk acknowledges the contributions from the FOM-Zeiss-ASML Industrial Partnership Programme CP3E. The authors would like to thank Prof. J. E. ten Elshof and Mr. Maarten Nijland for providing the nanosheets and Dr. Minh Nguyen for Pt coating.

\section{Bibliography}

[1] S. Trolier-McKinstry and P. Muralt, Thin film piezoelectrics for MEMS, Journal of Electroceramics 12, 7 (2004).

[2] Ferroelectric thin films, edited by M. Okuyama and Y. Ishibashi (SpringerVerlag, Berlin Heidelberg, 2005).

[3] B. Jaffe, W. R. Cook, and H. Jaffe, Piezoelectric ceramics (Academic, London, 1971).

[4] X.-h. Du, U. Belegundu, and K. Uchino, Crystal orientation dependence of piezoelectric properties in lead zirconate titanate: Theoretical expectation for thin films, Japanese Journal of Applied Physics 36, 5580 (1997).

[5] X.-h. Du, J. Zheng, U. Belegundu, and K. Uchino, Crystal orientation dependence of piezoelectric properties of lead zirconate titanate near the morphotropic phase boundary, Applied Physics Letters 72, 2421 (1998).

[6] L. X. Cao, Y. Xu, B. R. Zhao, L. P. Guo, L. Li, B. Xu, Y. Z. Zhang, H. Chen, A. J. Zhu, Z. H. Mai, J. H. Zhao, Y. F. Fu, and L. X. J, A structural investigation of high-quality epitaxial $\mathrm{Pb}(\mathrm{Zr}, \mathrm{Ti}) \mathrm{O}_{3}$ thin films, Journal of Physics D: Applied Physics 30, 1455 (1997).

[7] I. Vrejoiu, G. Le Rhun, L. Pintilie, D. Hesse, M. Alexe, and U. Gösele, Intrinsic ferroelectric properties of strained tetragonal $\mathrm{PbZr}_{0.2} \mathrm{Ti}_{0.8} \mathrm{O}_{3}$ obtained on layerbylayer grown, defectfree singlecrystalline films, Advanced Materials 18, 1657 (2006).

[8] G. Barucca, A. De Benedittis, A. Di Cristoforo, G. Majni, P. Mengucci, F. Leccabue, and B. E. Watts, Crystallisation of perovskite PZT films on $\mathrm{MgO}$ substrates, Thin Solid Films 319, 207 (1998).

[9] J. H. Kim and F. F. Lange, Epitaxial growth of $\mathrm{PbZr}_{0.5} \mathrm{Ti}_{0.5} \mathrm{O}_{3}$ thin films on (001) $\mathrm{LaAlO}_{3}$ by the chemical solution deposition method, Journal of Materials Research 14, 4004 (1999).

[10] M. Bayraktar, W. A. Wessels, C. J. Lee, F. A. van Goor, G. Koster, G. Rijnders, and F. Bijkerk, Active multilayer mirrors for reflectance tuning at extreme ultraviolet (EUV) wavelengths, Journal of Physics D: Applied Physics 45, 494001 (2012). 
[11] E. Louis, A. E. Yakshin, T. Tsarfati, and F. Bijkerk, Nanometer interface and materials control for multilayer EUV-optical applications, Progress in Surface Science 86, 255 (2011).

[12] D. K. Fork, D. B. Fenner, G. A. N. Connell, J. M. Phillips, and T. H. Geballe, Epitaxial yttria-stabilized zirconia on hydrogen-terminated Si by pulsed laser deposition, Applied Physics Letters 57, 1137 (1990).

[13] S. J. Wang, C. K. Ong, L. P. You, and S. Y. Xu, Epitaxial growth of yittriastabilized zirconia oxide thin film on natively oxidized silicon wafer without an amorphous layer, Semiconductor Science and Technology 15, 836 (2000).

[14] M. Dekkers, M. D. Nguyen, R. Steenwelle, P. M. te Riele, D. H. A. Blank, and G. Rijnders, Ferroelectric properties of epitaxial $\mathrm{Pb}(\mathrm{Zr}, \mathrm{Ti}) \mathrm{O}_{3}$ thin films on silicon by control of crystal orientation, Applied Physics Letters 95, 012902 (2009).

[15] A. Chopra, D. Pantel, Y. Kim, M. Alexe, and D. Hesse, Microstructure and ferroelectric properties of epitaxial cation ordered $\mathrm{PbSc}_{0.5} \mathrm{Ta}_{0.5} \mathrm{O}_{3}$ thin films grown on electroded and buffered Si(100), Journal of Applied Physics 114, 084107 (2013).

[16] J. G. E. Gardeniers, A. Smith, and C. Cobianu, Characterisation of sol-gel PZT films on Pt-coated substrates, Journal of Micromechanics and Microengineering 5, 153 (1995).

[17] A. Kumar, M. Alam, A. Mangiaracina, and M. Shamsuzzoha, Synthesis of the PZT films deposited on Pt-coated (100) Si substrates for nonvolatile memory applications, Journal of Electronic Materials 26, 1331 (1997).

[18] Y. Minemura, K. Nagasaka, T. Kiguchi, T. J. Konno, H. Funakubo, and H. Uchida, Fabrication and evaluation of one-axis oriented lead zirconate titanate films using metal-oxide nanosheet interface layer, Japanese Journal of Applied Physics 52, 09KA04 (2013).

[19] X. M. Lu, J. S. Zhu, X. F. Huang, C. Y. Lin, and Y. N. Wang, Laser-induced phase transformation from amorphous to perovskite in $\mathrm{PbZr}_{0.44} \mathrm{Ti}_{0.56} \mathrm{O}_{3}$ films with the substrate at room temperature, Applied Physics Letters 65, 2015 (1994).

[20] X. M. Lu, J. S. Zhu, W. S. Hu, Z. G. Liu, and Y. N. Wang, Pulsed excimer $(\mathrm{KrF})$ laser induced crystallization of $\mathrm{PbZr}_{0.44} \mathrm{Ti}_{0.56} \mathrm{O}_{3}$ amorphous films, Applied Physics Letters 66, 2481 (1995).

[21] S.-I. Kuroki, K. Tago, K. Kotani, and T. Ito, Low-temperature recrystallization of ferroelectric lead zirconate titanate thin films on glass substrate using continuous-wave green laser, Japanese Journal of Applied Physics 48, 04C142 (2009). 
[22] J. Jiang, S.-I. Kuroki, K. Kotani, and T. Ito, Ferroelectric properties of lead zirconate titanate thin film on glass substrate crystallized by continuous-wave green laser annealing, Japanese Journal of Applied Physics 49, 04DH14 (2010).

[23] S. S. Roy, H. Gleeson, C. P. Shaw, R. W. Whatmore, Z. Huang, Q. Zhang, and S. Dunn, Growth and characterisation of lead zirconate titanate (30/70) on indium tin oxide coated glass for oxide ferroelectric-liquid crystal display application, Integrated Ferroelectrics 29, 189 (2000).

[24] E. Bruno, F. Ciuchi, M. Castriota, S. Marino, G. Nicastro, E. Cazzanelli, and N. Scaramuzza, Structural transformations of PZT 53/47 sol-gel films on different substrates driven by thermal treatments, Ferroelectrics 396, 49 (2010).

[25] C. B. Eom, R. B. Van Dover, J. M. Phillips, D. J. Werder, J. H. Marshall, C. H. Chen, R. J. Cava, R. M. Fleming, and D. K. Fork, Fabrication and properties of epitaxial ferroelectric heterostructures with $\left(\mathrm{Sr} \mathrm{RuO}_{3}\right)$ isotropic metallic oxide electrodes, Applied Physics Letters 63, 2570 (1993).

[26] M.-S. Chen, T.-B. Wu, and J.-M. Wu, Effect of textured $\mathrm{LaNiO}_{3}$ electrode on the fatigue improvement of $\mathrm{Pb}\left(Z r_{0.53} \mathrm{Ti}_{0.47}\right) \mathrm{O}_{3}$ thin films, Applied Physics Letters 68, 1430 (1996).

[27] J. Lee, C. H. Choi, B. H. Park, T. W. Noh, and J. K. Lee, Built-in voltages and asymmetric polarization switching in $\mathrm{Pb}(\mathrm{Zr}, \mathrm{Ti}) \mathrm{O}_{3}$ thin film capacitors, Applied Physics Letters 72, 3380 (1998).

[28] C. W. Law, K. Y. Tong, J. H. Li, K. Li, and M. C. Poon, Effect of oxygen content and thickness of sputtered $\mathrm{RuO}_{x}$ electrodes on the ferroelectric and fatigue properties of sol-gel PZT thin films, Thin Solid Films 354, 162 (1999).

[29] P. Verardi, M. Dinescu, F. Craciun, R. Dinu, and M. F. Ciobanu, Growth of oriented $\mathrm{Pb}\left(Z r_{x} T i_{1-x}\right) O_{3}$ thin films on glass substrates by pulsed laser deposition, Applied Physics A 69, S837 (1999).

[30] R. H. T. Wilke, S. Trolier-McKinstry, P. B. Reid, and D. A. Schwartz, PZT piezoelectric films on glass for Gen-X imaging, Proceedings of SPIE 7803, 780300 (2010).

[31] D. H. Kim, Y. K. Kim, S. Hong, Y. Kim, and S. Baik, Nanoscale bit formation in highly (111)-oriented ferroelectric thin films deposited on glass substrates for high-density storage media, Nanotechnology 22, 245705 (2011).

[32] R. H. T. Wilke, R. L. Johnson-Wilke, V. Cotroneo, W. N. Davis, P. B. Reid, D. A. Schwartz, and S. Trolier-McKinstry, Sputter deposition of PZT piezoelectric films on thin glass substrates for adjustable x-ray optics, Applied Optics 52, 3412 (2013). 
[33] K. K. Uprety, L. E. Ocola, and O. Auciello, Growth and characterization of transparent $\mathrm{Pb}(\mathrm{Zi}, \mathrm{Ti}) \mathrm{O}_{3}$ capacitor on glass substrate, Journal of Applied Physics 102, 084107 (2007).

[34] Y. Yu, M. Lai, and L. Lu, Highly (100) oriented $\mathrm{Pb}\left(\mathrm{Zr}_{0.52} \mathrm{Ti}_{0.48}\right) \mathrm{O}_{3} / \mathrm{LaNiO}_{3}$ films grown on amorphous substrates by pulsed laser deposition, Applied Physics A 88, 365 (2007).

[35] K. Kikuta, K. Noda, S. Okumura, T. Yamaguchi, and S.-i. Hirano, Orientation control of perovskite thin films on glass substrates by the application of a seed layer prepared from oxide nanosheets, Journal of Sol-Gel Science and Technology 42, 381 (2007).

[36] J. Son and Y.-H. Shin, Highly c-Oriented $\mathrm{PbZr}_{0.48} \mathrm{Ti}_{0.52} \mathrm{O}_{3}$ Thin Films on Glass Substrates, Electrochemical and Solid-State Letters 12, G20 (2009).

[37] M. Nijland, S. Kumar, R. Lubbers, D. H. A. Blank, G. Rijnders, G. Koster, and J. E. ten Elshof, Local control over nucleation of epitaxial thin films by seed layers of inorganic nanosheets, ACS Applied Materials \& Interfaces 6, 2777 (2014).

[38] JCPDS card numbers are Pt: 87-0647, $\mathrm{LaNiO}_{3}$ : 33-0710 and $\mathrm{PbZr}_{0.52} \mathrm{Ti}_{0.48} \mathrm{O}_{3}:$ 56-0900.

[39] T. Rojac, M. Kosec, B. Budic, N. Setter, and D. Damjanovic, Strong ferroelectric domain-wall pinning in $\mathrm{BiFeO}_{3}$ ceramics, Journal of Applied Physics 108, 074107 (2010).

[40] P. Gerber, A. Roelofs, C. Kügeler, U. Böttger, R. Waser, and K. Prume, Effects of the top-electrode size on the piezoelectric properties ( $d_{33}$ and $S$ ) of lead zirconate titanate thin films, Journal of Applied Physics 96, 2800 (2004).

[41] S. Sivaramakrishnan, P. Mardilovich, A. Mason, A. Roelofs, T. SchmitzKempen, and S. Tiedke, Electrode size dependence of piezoelectric response of lead zirconate titanate thin films measured by double beam laser interferometry, Applied Physics Letters 103, 132904 (2013). 

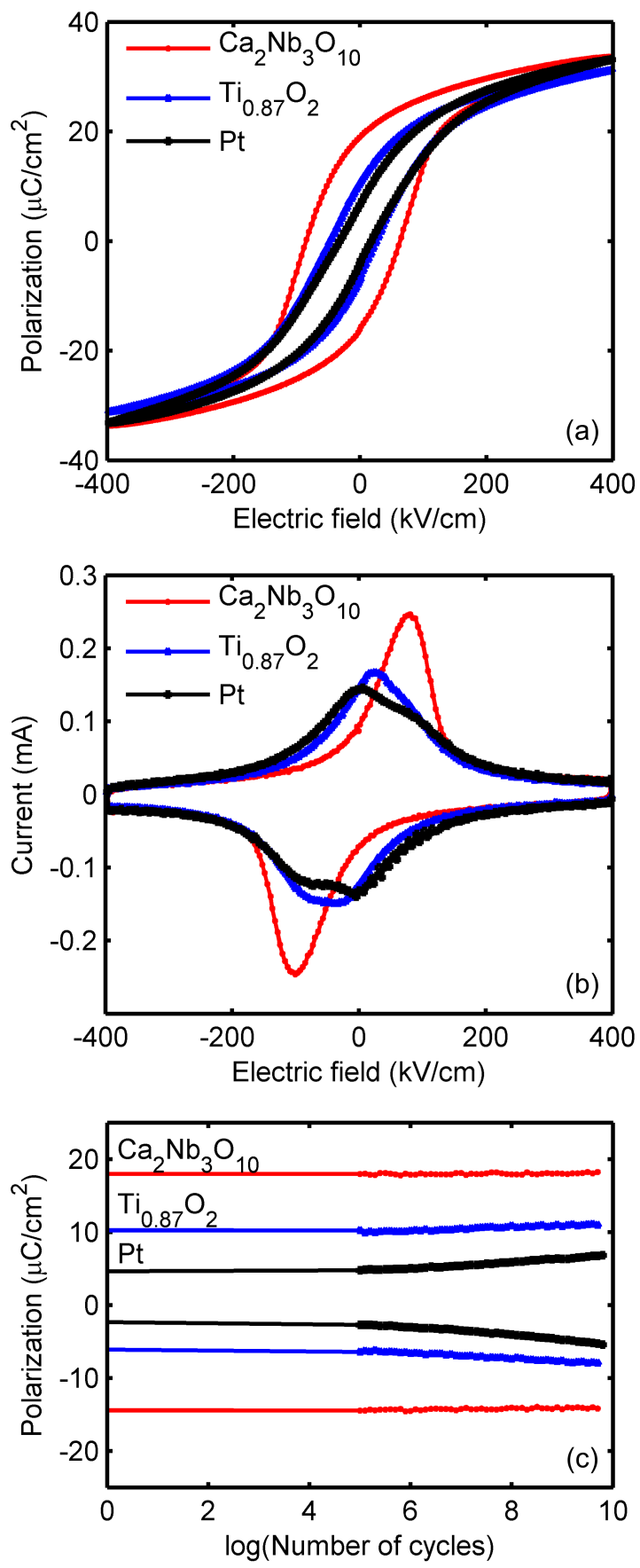

Figure 3.3: (a) Polarization-electric field hysteresis loops, (b) Current-electric field response, and (c) Remnant polarization- versus number of switching cycles. 


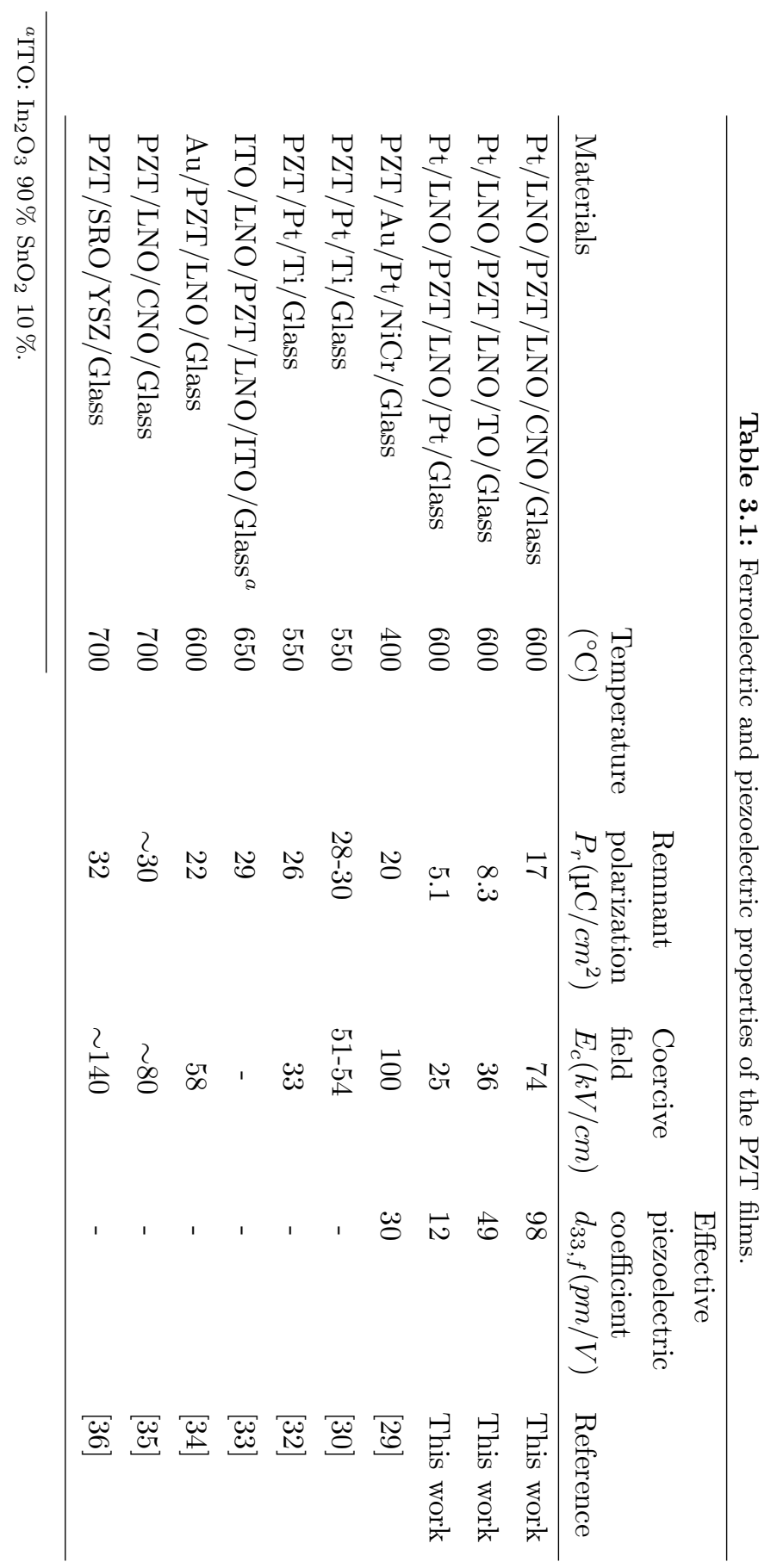




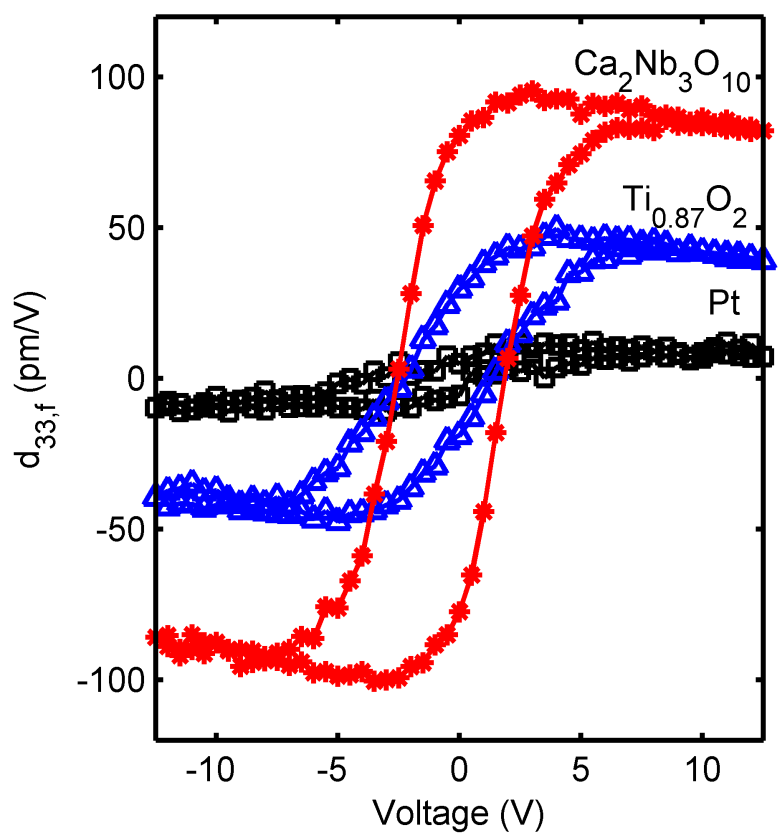

Figure 3.4: Longitudinal piezoelectric response $\left(d_{33, f}\right)$ of the three heterostructures measured using a laser Doppler vibrometer. 



\section{Tuning of large piezoelectric response in nanosheet-buffered $\mathrm{PbZr}_{0.52} \mathrm{Ti}_{0.48} \mathrm{O}_{3}$ films on glass substrates}

Renewed interest in utilizing the piezoelectric response of $\mathrm{PbZr}_{0.52} \mathrm{Ti}_{0.48} \mathrm{O}_{3}$ (PZT) thin films on glass substrates has been witnessed for a variety of applications, such as data storage and adaptive optics. These applications demand new methodologies for well-oriented growth of the PZT films and control of their piezoelectric response. Since such films are clamped to the substrate, the piezoelectric response is usually significantly reduced. Here, a novel method is presented to control the growth and enhance the piezoelectric response by minimizing the clamping effect of PZT films. Hierarchically ordered PZT columnar structures with preferred (100) orientation are achieved on glass substrates by utilizing crystalline nanosheets as seed layers. The lateral columnar density is tuned during the growth with pulsed laser deposition. For optimally tuned growth settings, the clamping effect is minimized and a large piezoelectric response of $280 \mathrm{pm} / \mathrm{V}$ is observed. To confirm that the clamping comprises the effective piezoelectric response, dense films are deposited using a lower deposition rate and a $\mathrm{BiFeO}_{3}$ buffer layer, which resulted in significantly lower piezoelectric responses. This paper demonstrates a novel method to integrate PZT films on glass substrates and achieve a large piezoelectric response. 


\subsection{Introduction}

Perovskite oxides form a special and exciting class of materials which exhibit diverse and exotic multifunctional physical properties such as superconductivity, photovoltaic effect, colossal magnetoresistance, ferroelectricity and piezoelectricity [1-5]. In particular, there has been an intensive research in harvesting the piezoelectric response of ferroelectric thin films for a wide range of microelectromechanical systems (MEMS) devices as sensors and actuators [6-9]. Recently, a growing interest has been witnessed in integrating ferroelectric thin films on amorphous structures or glass substrates for applications in data storage, electronic displays and adaptive optics [10-17]. These applications strongly demand high and stable piezoelectric response for fabrication of more efficient devices.

The piezoelectric response in thin films is known to depend on composition, growth quality, orientation and size of the fabricated devices [7, 18-21]. Among all the ferroelectric materials $\mathrm{PbZr}_{x} \mathrm{Ti}_{1-x} \mathrm{O}_{3}$ with morphotropic phase boundary composition, $\mathrm{PbZr}_{0.52} \mathrm{Ti}_{0.48} \mathrm{O}_{3}$ (PZT), is usually preferred not only for its high piezoelectric response, but also for a large remnant polarization and low coercive electric field [5, 9, 22, 23]. Therefore growth of well-oriented crystalline or epitaxial PZT films on glass substrates with control on the orientation is highly desired. Achieving epitaxial growth with (001) orientation is known to maximize the piezoelectric response compared to the polycrystalline films [18, 19]. However the piezoelectric response is still drastically reduced compared to bulk PZT materials due to clamping of the thin film by the substrate [24-31]. On application of an electric field in the direction normal to the PZT film surface (longitudinal direction), the longitudinal expansion of the PZT film is coupled to a contraction in the direction parallel to its surface (transverse direction). Since the transverse contraction is constrained by the substrate, the effective longitudinal piezoelectric response of the PZT film is significantly reduced.

The clamping effect is less pronounced for structures that have smaller lateral dimensions, thus a lot of research has been directed to fabricate island-like nanostructures with same lateral size as that of the thickness of the film to enhance the piezoelectric response [21, 32-35]. However requirement of additional processing steps such as focused ion beam milling and chemical etching to pattern these islands increase the risk of contamination and damage. Most importantly reduced piezoelectric response of the island nanostructures with large lateral dimensions, where the clamping effect is most dominant, still remains as an issue.

Here we report on hierarchically ordered columnar growth of crystalline PZT films with preferred (100) orientation on glass substrates and minimization of the clamping effect by tuning the growth conditions. Local epitaxial growth and control on growth orientation was achieved by utilizing crystalline nanosheets as buffer layers on the glass substrates [36]. $\mathrm{LaNiO}_{3}$ (LNO) electrodes and the PZT films were grown on the nanosheet buffered glass substrates using pulsed laser deposition (PLD). PLD is a revolutionary technique to grow high quality multifunctional oxide thin films and allows precise control on the growth quality by tuning of the deposition parameters such as laser fluence, repetition rate of laser pulses, growth temperature and background gas pressure [20, 37-39]. Most 
important for this work, is the ability to increase the growth speed, by increasing the repetition rate of the laser pulses. While the amount of deposited material within one laser pulse stays the same, the repetition rate will determine the overall growth speed. In PLD of PZT, this will not affect the composition of the thin film. In our approach, we noticed a dramatic change in the columnar growth of the PZT films, and significantly reduced clamping effect.

\subsection{Experimental methods}

In order to promote crystalline growth and control on the growth orientation of the subsequent layers, $\mathrm{Ca}_{2} \mathrm{Nb}_{3} \mathrm{O}_{10}$ (CNO) nanosheets were deposited on glass substrates. The CNO nanosheets were exfoliated by chemical processing from their layer-structure parent compound $\mathrm{KCa}_{2} \mathrm{Nb}_{3} \mathrm{O}_{10}$. In this process, the parent compound material $\mathrm{KCa}_{2} \mathrm{Nb}_{3} \mathrm{O}_{10}$ was first treated with nitric acid to obtain a protonated compound which was exfoliated to CNO nanosheets on further treatment with exfoliation agent tetrabutylammonium hydroxide. The exfoliated nanosheets were transferred to the glass substrates using Langmuir-Blodgett deposition process at room temperature. The glass substrates used in this article were of $500 \mu \mathrm{m}$ thickness. Prior to transfer of the nanosheets, the glass substrates were first cleaned on a hot plate at $250{ }^{\circ} \mathrm{C}$ with a jet of supercritical $\mathrm{CO}_{2}$ followed by oxygen plasma cleaning. More experimental details of the nanosheet preparation and deposition to glass substrates can be found elsewhere [40].

The nanosheet coated glass substrate was loaded into the PLD chamber and a base pressure of $5 \times 10^{-7}$ mbar was maintained before raising the substrate temperature. The nanosheet coated substrate was annealed at $600{ }^{\circ} \mathrm{C}$ for 60 minutes in 0.140 mbar oxygen pressure. All the oxide layers on nanosheet coated glass substrate were deposited in situ by ablating materials from their respective stoichiometric targets using PLD with a KrF excimer laser operating at $248 \mathrm{~nm}$ wavelength with a pulse duration of $20 \mathrm{~ns}$. The following three heterostructures (named $\mathrm{H}_{1}, \mathrm{H}_{2}$ and $\mathrm{H}_{3}$ hereafter) were deposited with different repetition rates of the laser pulses. Within $\mathrm{H}_{3}$, a $\mathrm{BiFeO}_{3}$ buffer layer is used:

$$
\begin{aligned}
& \mathrm{H}_{1}: \operatorname{LNO}(5 \mathrm{~Hz}) / \mathbf{P Z T}(\mathbf{5} \text { and } \mathbf{2 0 ~ H z}) / \mathrm{LNO}(5 \mathrm{~Hz}) / \mathrm{CNO} / \text { Glass, } \\
& \mathrm{H}_{2}: \mathrm{LNO}(5 \mathrm{~Hz}) / \mathbf{P Z T}(\mathbf{5 ~ H z}) / \operatorname{LNO}(5 \mathrm{~Hz}) / \mathrm{CNO} / \text { Glass, } \\
& \mathrm{H}_{3}: \operatorname{LNO}(5 \mathrm{~Hz}) / \mathbf{P Z T}(\mathbf{5 ~ H z}) / \mathrm{BiFeO}_{3}(5 \mathrm{~Hz}) / \mathrm{LNO}(5 \mathrm{~Hz}) / \mathrm{CNO} / \text { Glass. }
\end{aligned}
$$

Electrical measurements were facilitated using the LNO bottom and top electrodes. The LNO electrodes were deposited at $600{ }^{\circ} \mathrm{C}$ with a laser fluence and repetition rate of $1.5 \mathrm{~J} / \mathrm{cm}^{2}$ and $5 \mathrm{~Hz}$, respectively. For the $\mathrm{PZT}$ layer, $\mathrm{PbZr}_{0.52} \mathrm{Ti}_{0.48} \mathrm{O}_{3}$ morphotropic phase boundary (MPB) composition was used to harvest the highest piezoelectric response. PZT films were deposited at $585{ }^{\circ} \mathrm{C}$ in $0.270 \mathrm{mbar}$ oxygen pressure with a laser fluence of $2 \mathrm{~J} / \mathrm{cm}^{2}$. During deposition of the PZT layer for the first heterostructure $\left(\mathrm{H}_{1}\right)$, a dense PZT seed layer $(\sim 50 \mathrm{~nm})$ was deposited with $5 \mathrm{~Hz}$ repetition rate to avoid the short circuiting between the top and bottom electrodes. Then the repetition rate was switched to $20 \mathrm{~Hz}$ for the 
rest of the deposition. The $\mathrm{PZT}$ films were deposited with $5 \mathrm{~Hz}$ repetition rate completely for both $\mathrm{H}_{2}$ and $\mathrm{H}_{3}$ heterostructures. A $\mathrm{BiFeO}_{3}(\mathrm{BFO})$ buffer layer was deposited with the same growth conditions as LNO layers for $\mathrm{H}_{3}$ heterostructure. A $100 \mathrm{~nm}$ thick platinum (Pt) layer was sputtered using radio frequency sputtering at room temperature on the top LNO electrode for all the heterostructures. The Pt layer deposited at the top improves the homogeneity of the electric field across the top electrode. Top electrodes with $200 \times 200 \mu \mathrm{m}^{2}$ area were patterned using a standard photolithography process and structured by dry argon etching.

The crystallographic properties of the samples was analyzed using an x-ray diffractometer (Philips X'Pert MRD) with $\mathrm{Cu}-\mathrm{K} \alpha$ radiation. Pole figure maps were generated from electron back scattering diffraction (EBSD) patterns of the PZT films recorded using a high resolution scanning electron microscope (HRSEM, Zeiss MERLIN). The microstructure of the samples was analyzed by using transmission electron microscopy (TEM, Jeol 4010 operating at $400 \mathrm{kV}$ ) measurements. Mechanical and ion-beam etching based standard techniques were employed for sample preparation for TEM analysis. The fatigue measurements were recorded using a ferroelectric tester ( $\mathrm{TF}$ analyzer 2000, aixACCT). Fatigue measurements were performed using fatigue pulses of $15 \mathrm{~V}$ amplitude with a frequency of $100 \mathrm{kHz}$. After each fatigue cycle, the $P-E$ hysteresis loop was measured with a triangular pulse of $30 \mathrm{~V}$ amplitude at $1 \mathrm{kHz}$ frequency. The macroscopic piezoelectric responses of the films were measured using a laser Doppler vibrometer (LDV, Polytec MSA-400) operating at $8 \mathrm{kHz}$. To carry out the LDV measurements, all the samples were glued to a large metal plate with silver paste to impede the bending of the substrates. The LDV measurements were performed by applying a small AC electric field $(2.5 \mathrm{kV} / \mathrm{cm})$ superimposed on a DC voltage sweeping from $-12.5 \mathrm{~V}$ to $+12.5 \mathrm{~V}$.

\subsection{Results and discussion}

The XRD patterns reveal a predominantly $(100)_{\mathrm{pc}}$ oriented PZT growth as shown in Fig. 4.1 (shown only for $\mathrm{H}_{1}$ ). The subscript "pc" stands for pseudo-cubic indexing which is used for all the materials in this paper. However a minor $(110)_{\mathrm{pc}}$ reflection, which is two orders of magnitude smaller than the $(100)_{\mathrm{pc}}$ peak, is also observed. The PZT films for all the three heterostructures were found to have a pure perovskite phase without the presence of any impurity or pyrochlore phase. Only $(100)_{\mathrm{pc}}$ orientation was observed for the LNO electrodes. It is also worth noting here that PZT films were also found in purely (100)orientation up to $750 \mathrm{~nm}[36]$. The $(100)_{\mathrm{pc}}$ oriented growth of the LNO bottom electrode is facilitated by the match of its lattice parameters to the underlying CNO nanosheets as schematically illustrated in Fig. 4.2(a) and (b). The CNO nanosheets are known to have a $2 \mathrm{D}$ square lattice with a lattice parameter of $a_{\mathrm{CNO}}=3.86 \AA$ which matches the in-plane lattice parameter of the LNO pseudocubic unit-cell as shown in the schematic in Fig. 4.2(b) [40, 41]. In this study, the lateral size of $\mathrm{CNO}$ nanosheets deposited on glass substrate were around $\sim 2 \mu \mathrm{m}$ 
as can be seen in Appendix B [40]. Pure $(100)_{\mathrm{pc}}$ oriented growth of the LNO bottom electrode facilitates a $(100)_{\mathrm{pc}}$ oriented growth of the subsequent PZT layer. In addition to the out-of-plane characterization by XRD, the in-plane as well as out-of-plane crystal orientation of the PZT film for $\mathrm{H}_{1}$ was mapped using EBSD technique. The generated out-of-plane and in-plane pole figure maps are shown in Fig. 4.3(a) and (b), respectively. The out-of-plane pole figure map in Fig. 4.3(a) demonstrates a dominant $(100)_{\mathrm{pc}}$ orientation (above $99 \%$ of the measured area) as the whole map has a single color (red color representing $(100)_{\mathrm{pc}}$ orientation). A minor amount of $(110)_{\mathrm{pc}}$ orientation (encircled region that has green color representing (110) orientation) is also observed, which is indeed in accordance to our XRD observations. The in-plane pole figure map in Fig. 4.3(c) consists of predominately two different colors representing the $(100)_{\mathrm{pc}}$ and $(110)_{\mathrm{pc}}$ orientations which signifies that the PZT film is randomly oriented in-plane. This in-plane random orientation of the PZT film is directly linked to the random inplane orientation of the CNO nanosheets lying underneath [40].

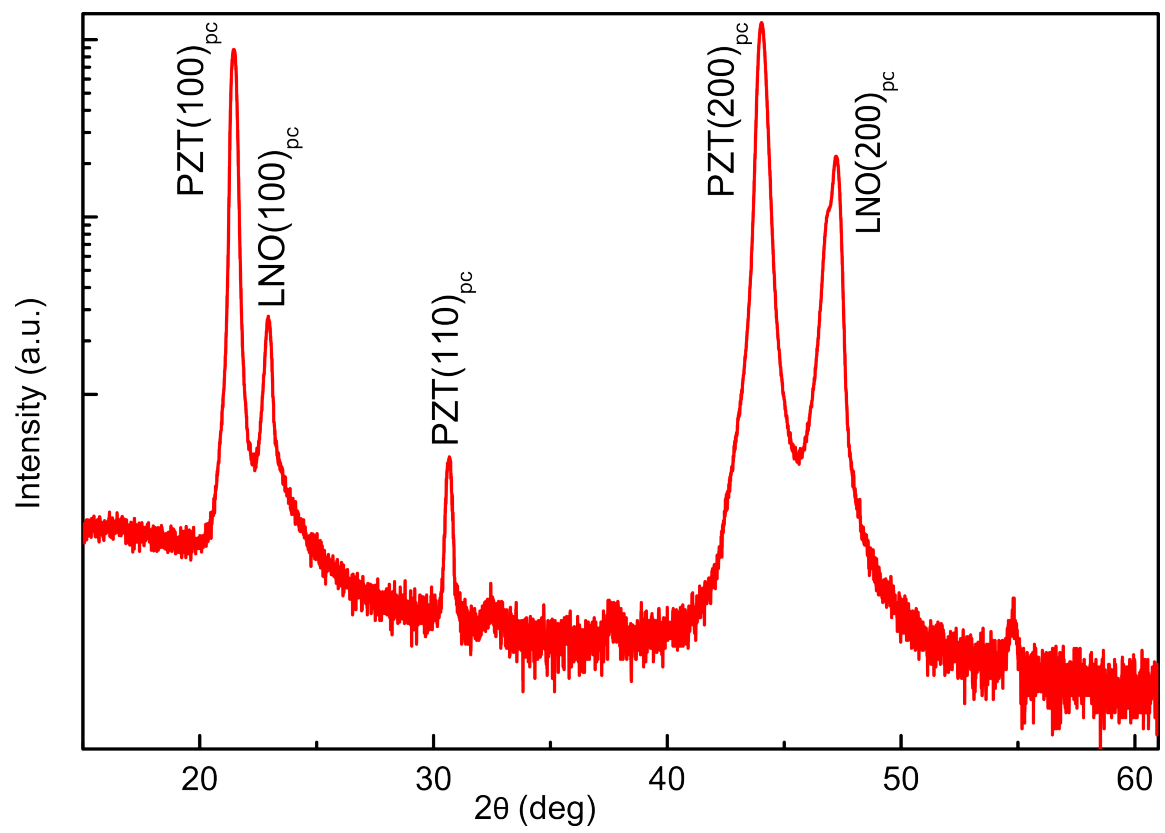

Figure 4.1: XRD $\theta-2 \theta$ scan of the LNO / PZT (5 and $20 \mathrm{~Hz}) /$ LNO / CNO / Glass heterostructure (subscript "pc" stands for pseudocubic indexing).

Detailed internal microstructure and thickness investigations were performed using TEM. A cross-sectional TEM image of a $2 \mu \mathrm{m}$ thick PZT film on a $200 \mathrm{~nm}$ thick LNO bottom electrode for the $\mathrm{H}_{1}$ heterostructure is shown in Fig. 4.4(a). The thickness of the top LNO and Pt electrodes were both found to be $\sim 100 \mathrm{~nm}$. A magnified image was captured in order to analyze the interface quality between the glass substrate and nanosheets as shown in Fig. 4.4(b). A sharp and abrupt interface is visible between the glass and nanosheets which confirms the effective- 


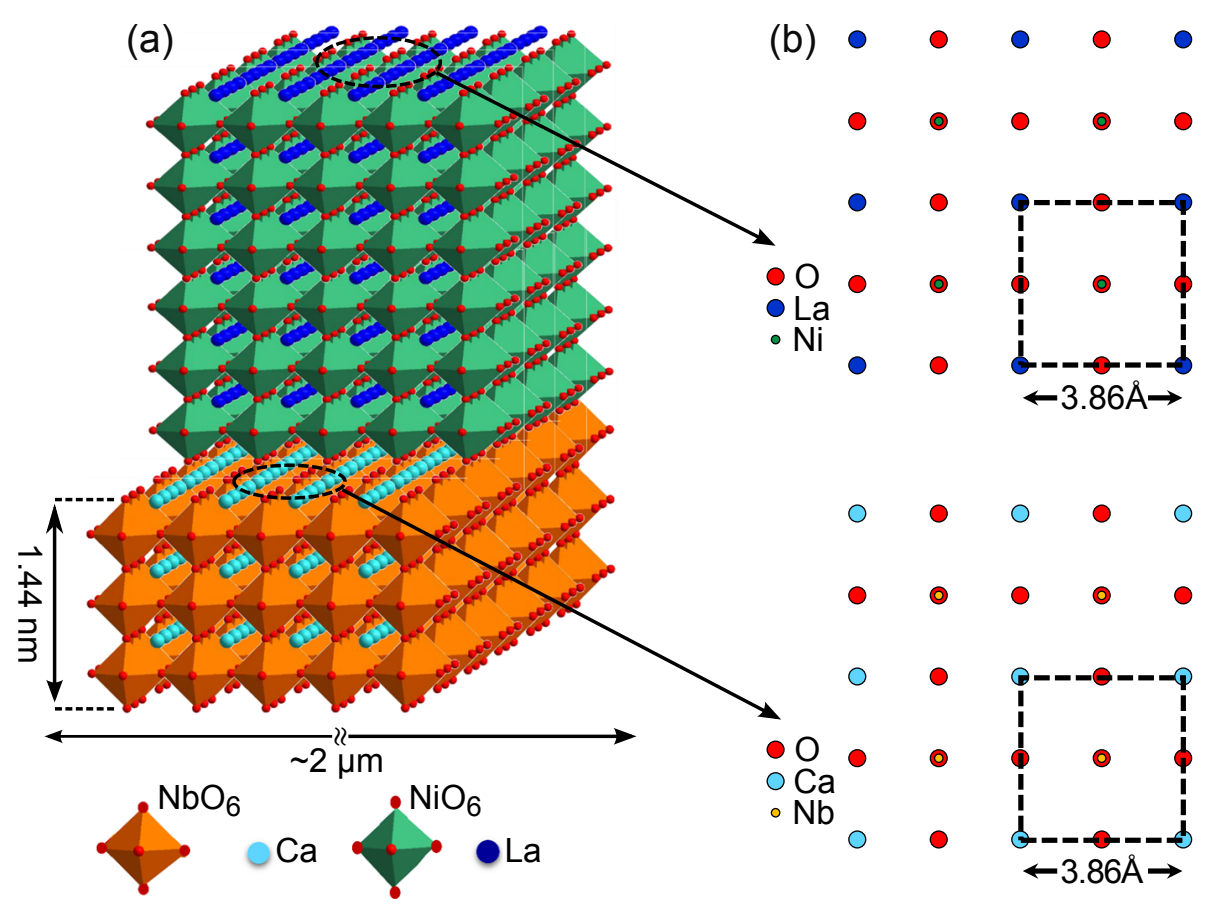

Figure 4.2: A schematic illustration of (a) growth of $\mathrm{LaNiO} 3$ perovskite on a perovskite-related $\mathrm{Ca}_{2} \mathrm{Nb}_{3} \mathrm{O}_{10}$ nanosheet, (b) the square in-plane lattice of the $\mathrm{LaNiO}_{3}$ and $\mathrm{Ca}_{2} \mathrm{Nb}_{3} \mathrm{O}_{10}$. The ideal fitting of the lattice parameters resulting in $(100)_{p c}$ growth.

ness of using nanosheets as buffer layer to grow a high quality crystalline LNO layer on an amorphous glass substrate. Further analysis of the microstructure for $\mathrm{H}_{1}$ heterostructure reveals that the PZT film can be distinctively identified into two regions along the growth direction as: 1) region 1 at the bottom where the PZT film is dense and has continuous columns, 2) region 2 at the top where the PZT film has a laterally seperated columnar structure. This difference in the microstructure of the PZT film was controlled by changing the repetition rate of the laser pulses. As discussed in the experimental section, first $200 \mathrm{~nm}$ of the PZT film was grown with a $5 \mathrm{~Hz}$ repetition rate which resulted in densely packed continuous columnar growth as seen in region 1. After ensuring the full coverage of LNO bottom electrode with $5 \mathrm{~Hz}$ repetition rate, the repetition rate was increased to $20 \mathrm{~Hz}$ which resulted in less densely packed columnar growth as in region 2. Thus, just by changing the repetition rate during the growth, we controlled the lateral density of the columnar growth. On magnifying the seperated columnar region (region 2) as shown in Fig. 4.4(c), distinct columns can be seen with lateral sizes around $100 \mathrm{~nm}$ can be seen. The crystallography of these columns was analyzed using selected area electron diffraction (SAED). The SAED pattern for one of the columns that is shown in Fig. 4.4(d)confirms an epitaxial growth. 


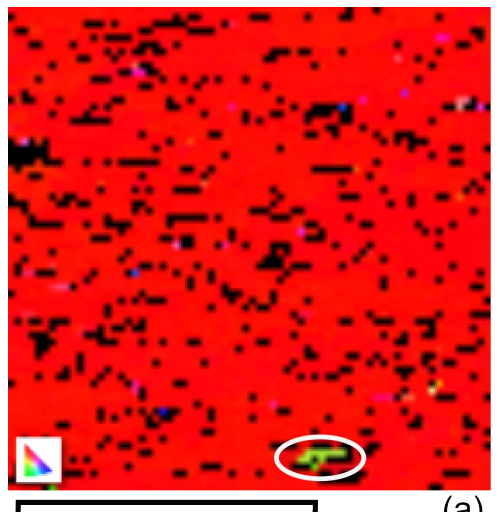

$5 \mu \mathrm{m}$

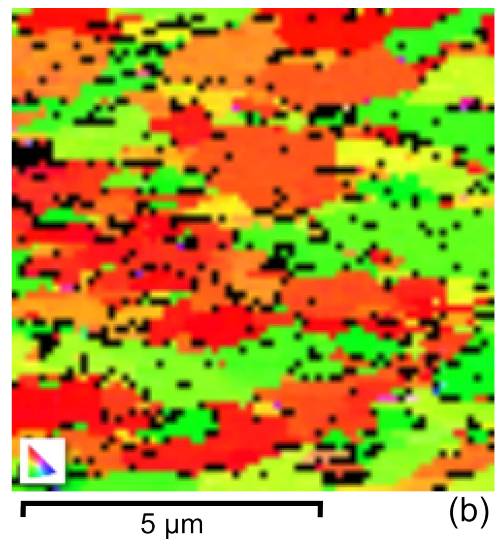

(b)

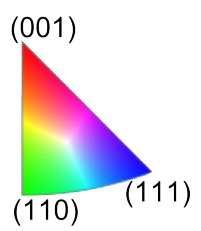

$(110)$

Figure 4.3: (a) Out-of-plane and (b) in-plane pole figure map of the PZT film generated using electron backscatter diffraction measurements.

The mechanism behind such a columnar growth can be explained by diffusivity of the adatoms impinging to the substrate from vapor phase during the PLD process. The effect of the diffusivity of the adatoms in tailoring the type of growth is widely studied and well reported in the literature [37-39]. Low diffusivity of adatoms manifests into a columnar growth separated from each other a few nanometers. To confirm this, the microstructure of PZT films deposited with $5 \mathrm{~Hz}$ repetition rate for both $\mathrm{H}_{2}$ and $\mathrm{H}_{3}$ heterostructures were investigated using TEM as shown in Fig. 4.5(a) and (b), respectively. TEM investigations demonstrate a denser and more continuous growth of PZT for the $\mathrm{H}_{2}$ heterostructure $(5 \mathrm{~Hz}$ repetition rate) as compared to $\mathrm{PZT}$ growth for the $\mathrm{H}_{1}$ heterostructure $(5 \mathrm{~Hz}$ and $20 \mathrm{~Hz}$ repetition rate). This validates our consideration of the link between the sparse columnar growth and the high repetition rate of laser pulses.

The role of lattice-mismatch in controlling the quality of the films is well studied $[20,37,38]$. The lattice parameters for the pseudo-cubic unit cells of the LNO and PZT layers are $a_{\mathrm{LNO}, \mathrm{pc}}=3.86 \AA$ and $a_{\mathrm{PZT}, \mathrm{pc}}=4.06 \AA$, respectively. The lattice mismatch between the PZT and LNO is $5.18 \%\left[\left(a_{\mathrm{PZT}, \mathrm{pc}} / a_{\mathrm{LNO}, \mathrm{pc}}-1\right) \times 100\right]$ which resulted in a dense columnar growth as observed for PZT in $\mathrm{H}_{2}$. In order to further increase the density of columnar structures, a $50 \mathrm{~nm}$ thick BFO layer was used as a buffer layer between the LNO and PZT films. The pseudo-cubic unit cell of BFO has a lattice parameter of $a_{\mathrm{BFO}, \mathrm{pc}}=3.96 \AA$ which reduces the lattice mismatch from $5.18 \%$ (PZT and LNO) to $2.52 \%$ (between PZT and BFO) and hence promotes a denser PZT growth as evident from Fig. 4.5(b). All in all, the TEM images in Fig. 4.4 and 4.5 demonstrate that the columnar density of the PZT film is dramatically influenced by the deposition parameters and can be tuned either by controlling the repetition rate of the laser pulses or by using suitable buffer layers.

The measured longitudinal piezoelectric responses of the PZT films $\left(d_{33, \mathrm{f}}\right)$ are shown in Fig. 4.6(a). A large effective piezoelectric coefficient of $280 \mathrm{pm} / \mathrm{V}$ was 

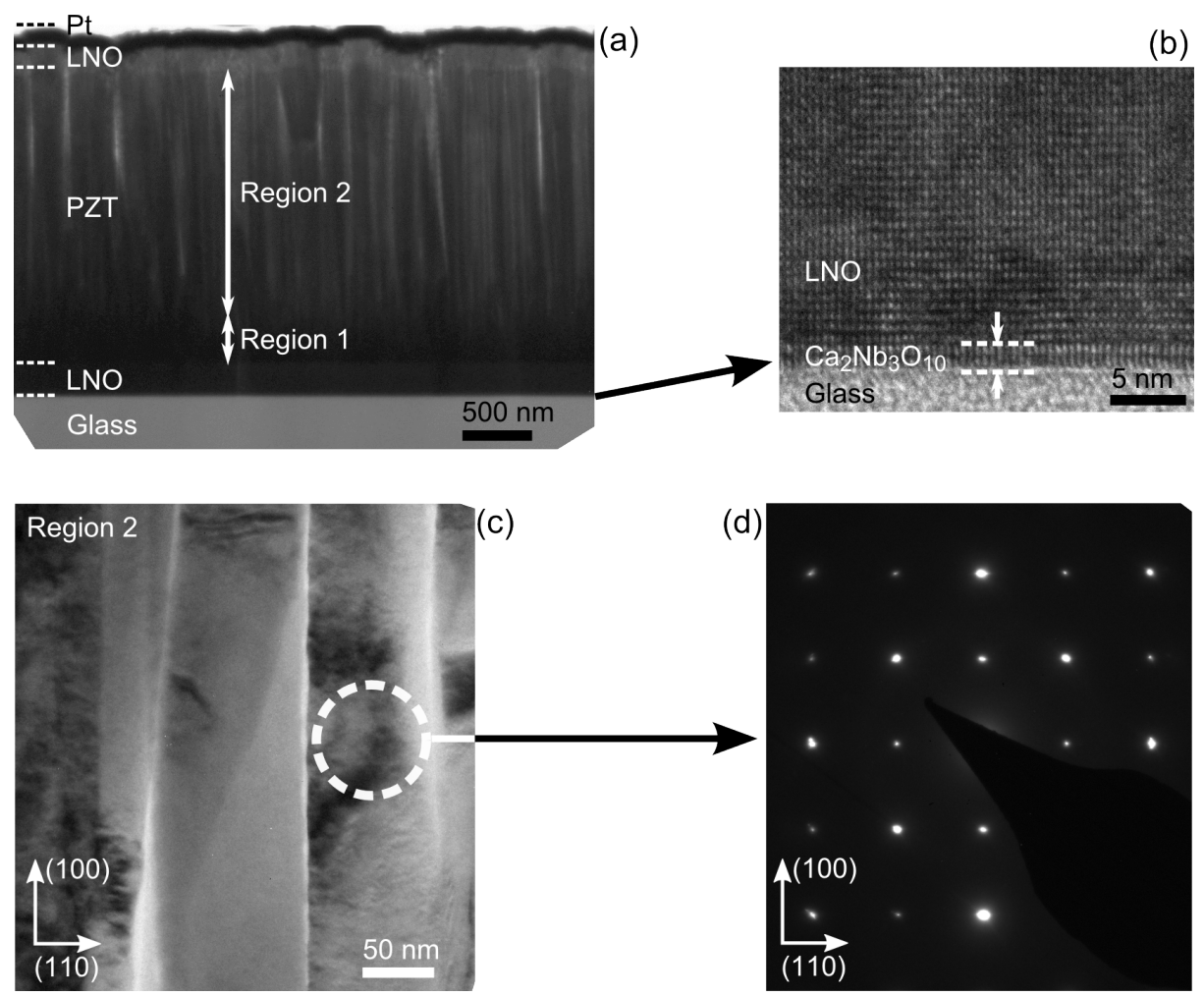

Figure 4.4: (a) Cross-sectional TEM image of the first heterostructure $\left(\mathrm{H}_{1}\right.$ : $\mathrm{Pt} / \mathrm{LNO} / \mathrm{PZT} / \mathrm{LNO} / \mathrm{CNO} /$ Glass $)$ in which PZT layer was first deposited at $5 \mathrm{~Hz}$ repetition rate (region 1 with dense packing) and then $20 \mathrm{~Hz}$ repetition rate (region 2 with seperated columns). (b) A magnified image of $\mathrm{Ca}_{2} \mathrm{Nb}_{3} \mathrm{O}_{10}$ nanosheet and glass interface. (c) A magnified image of the columns from region 2 of the PZT film. (d) SAED pattern recorded for one of the column confirming an epitaxial growth.

observed for the $\mathrm{H}_{1}$ heterostructure with seperated columns. However, much lower piezoelectric coefficients of $140 \mathrm{pm} / \mathrm{V}$ and $50 \mathrm{pm} / \mathrm{V}$ were measured for the $\mathrm{H}_{2}$ and $\mathrm{H}_{3}$ heterostructures with denser columns, respectively. We attribute this large decrease in the effective piezoelectric response to the increase in the clamping conditions affected by the columnar density. Although this trend seems paradoxical considering previous reports of piezoelectric response in bulk materials wherein longitudinal piezoelectric response has been shown to decrease with decreasing density [42-44]. For example in Ref. [42] bulk PZT materials has been prepared with decreasing densities as listed in Table 4.1. The density decreases from $7982 \mathrm{~g} / \mathrm{cm}^{3}$ to $6334 \mathrm{~g} / \mathrm{cm}^{3}$ and correspondingly the piezoelectric coefficient $d_{33}$ also decreases from $451 \mathrm{pm} / \mathrm{V}$ to $350 \mathrm{pm} / \mathrm{V}$. A closer look using finite element modeling (FEM) reveals that a decrease in film density results in an increase in the effective piezoelectric response of the thin films due to the 


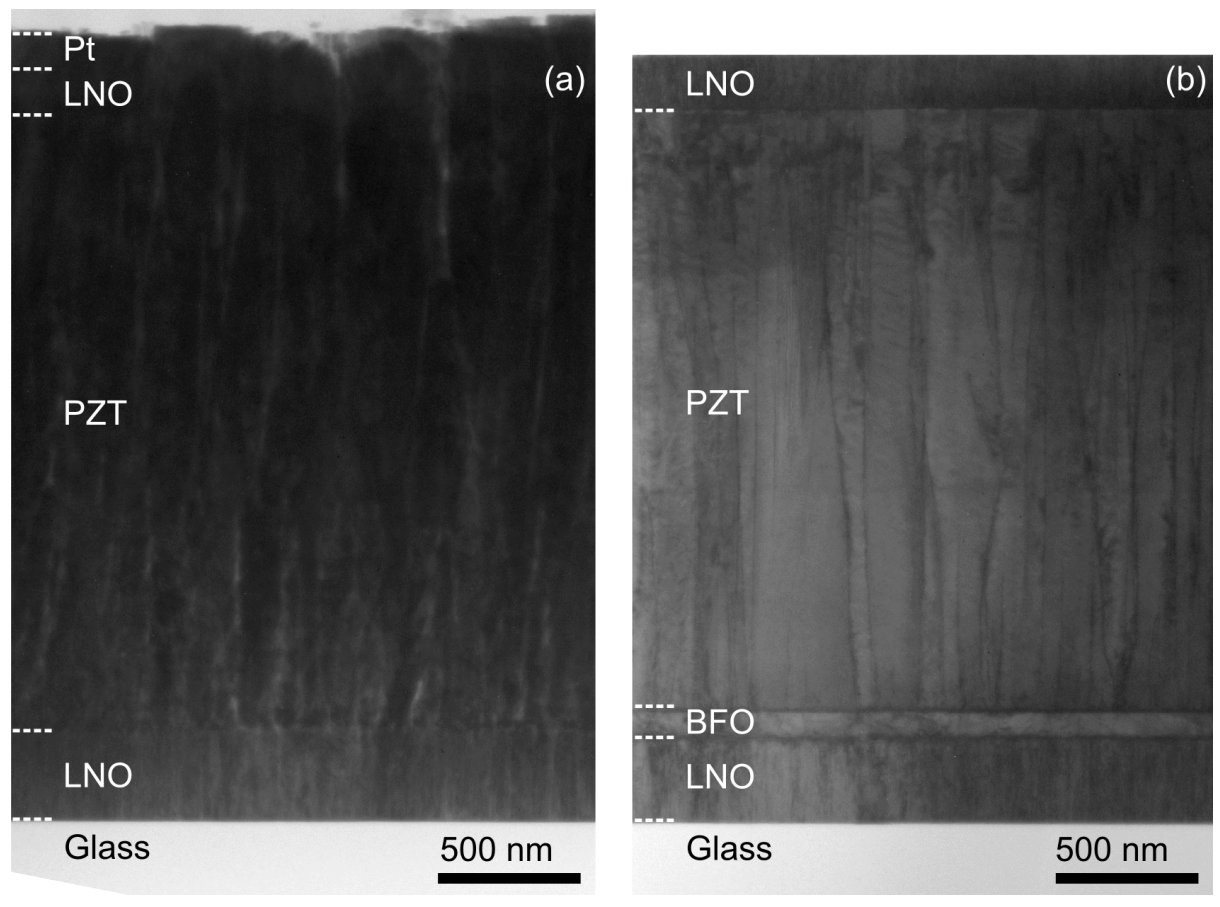

Figure 4.5: Cross-sectional TEM image of the heterostructures deposited at $5 \mathrm{~Hz}$ rate (a) $\mathrm{H}_{2}: \mathrm{Pt} / \mathrm{LNO} / \mathrm{PZT} / \mathrm{LNO} / \mathrm{CNO} /$ Glass, (b) $\mathrm{H}_{3}: \mathrm{LNO} / \mathrm{PZT} / \mathrm{BFO} / \mathrm{LNO} / \mathrm{CNO} /$ Glass.

boundary conditions and film/substrate dimensions [45].

We used FEM to demonstrate that a small decrease in density results in a large increase in the effective piezoelectric response in thin film form. In the FEM, the surface displacement of the PZT film is calculated using the constitutive equations and boundary conditions. We take the calculated surface displacement per unit volt as the effective piezoelectric coefficient $\left(d_{33, f}^{F E M}\right)$. In modeling, the sample dimensions and boundary conditions similar to our LDV measurement were used. The piezoelectric material is assumed to be isotropic and its density $(\rho)$, elastic compliance coefficients $\left(s_{11}, s_{12}=s_{13}\right)$, dielectric constant $\left(\varepsilon_{33}^{T} / \varepsilon_{0}\right)$ and piezoelectric coefficients $\left(d_{31}, d_{33}\right)$ are derived from Ref. [42] and presented in Table 4.1. The density and the compliance parameters of the substrate are derived from Ref. [46]. The density and bulk piezoelectric coefficient $d_{33}$ decreases by about the same ratio of $20 \%$ as shown in Table 4.1 . In contrast, the effective piezoelectric coefficient calculated using FEM increases drastically by $190 \%$ (from $100 \mathrm{pm} / \mathrm{V}$ to $288 \mathrm{pm} / \mathrm{V}$ ). Effectively, the small decrease in the density is amplified by the transverse piezoelectric coefficient $d_{31}$ and constraints of the thin film, which results in a large increase in the effective piezoelectric response as observed in our PZT films. The effective piezoelectric response of PZT films was enhanced by $460 \%$ as the density of the columnar structures was varied from 
densest structure $\mathrm{H}_{3}(50 \mathrm{pm} / \mathrm{V})$ to the separated structure $\mathrm{H}_{1}(280 \mathrm{pm} / \mathrm{V})$ due to reduced density and substrate clamping. This effective piezoelectric coefficient of $280 \mathrm{pm} / \mathrm{V}$ is also, to the best of our knowledge, the highest piezoelectric coefficient measured on glass substrates [36, 47].
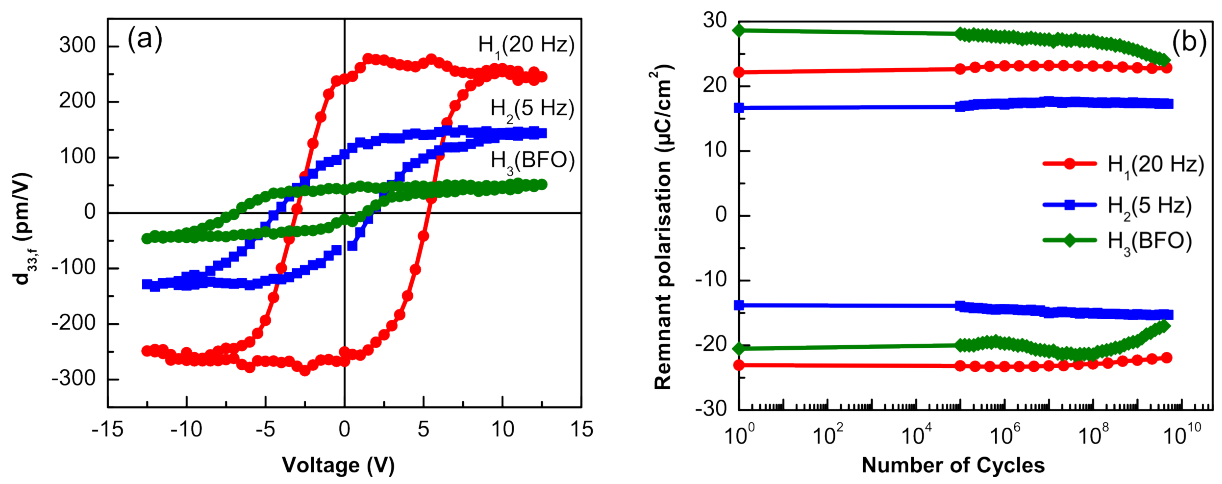

Figure 4.6: (a) Longitudinal piezoelectric response $\left(d_{33, \mathrm{f}}\right)$ of the three heterostructures measured using a laser Doppler vibrometer. (b) A plot of remnant polarization versus number of switching cycles.

Furthermore, for device applications of these films, a long-term switching stability on applting external electric field is essential. The stable operation of PZT films for all the three heterostructures was confirmed using fatigue measurements as shown in Fig. 4.6(b). Up to the tested $10^{9}$ operating cycles, the remnant polarization of the heterostructures $\mathrm{H}_{1}$ and $\mathrm{H}_{2}$ are stable, which demonstrate the applicability of these films in device applications for future technology. For the heterostructure $\mathrm{H}_{3}$ a decrease in the remnant polarization is observed after $10^{8}$ cycles possibly due to BFO interface.

\subsection{Conclusions}

In summary, we demonstrated a novel approach to tune the piezoelectric response of PZT films by controlling the lateral density of hierarchically ordered columns without any chemical treatment. The columnar density was controlled either by changing the repetition rate of PLD process or by introducing a suitable BFO buffer layer. PZT films deposited with $20 \mathrm{~Hz}$ repetition rate showed seperated columns which manifested in a large piezoelectric response of $280 \mathrm{pm} / \mathrm{V}$ due to reduced substrate induced clamping. In case of films deposited with $5 \mathrm{~Hz}$ repetition rate either directly on $\mathrm{LNO}$ or on BFO buffered samples, densely packed columnar growth resulted in a reduced piezoelectric response which is conclusively demonstrated the effect of the substrate clamping. To conclude, this work offers new possibilities to tune the piezoelectric response without any chemical treatment, which opens new avenues in thin film fabrication for the 
future device applications. The same approach can be extended to other oxide systems as well to tune their response.

\section{Acknowledgements}

Authors M. Bayraktar and A. Chopra both contributed equally to this article. This research program is funded by "Stichting Technologie en Wetenschap (STW)" under the contract 10448 with the project name "Smart Multilayer Interactive Optics for Lithography at Extreme UV wavelengths (SMILE)". The authors would like to thank Prof. J. E. ten Elshof and Mr. Maarten Nijland for providing the nanosheets and Dr. Minh Nguyen for Pt coating.

\section{Bibliography}

[1] B. Baumert, Barium potassium bismuth oxide: A review, Journal of Superconductivity 8, 175 (1995).

[2] M. Alexe and D. Hesse, Tip-enhanced photovoltaic effects in bismuth ferrite, Nature Communications 2, 256 (2011).

[3] S. Jin, T. H. Tiefel, M. McCormack, R. A. Fastnacht, R. Ramesh, and L. H. Chen, Thousandfold change in resistivity in magnetoresistive La-Ca-Mn-O films, Science 264, 413 (1994).

[4] I. Vrejoiu, G. Le Rhun, L. Pintilie, D. Hesse, M. Alexe, and U. Gösele, Intrinsic ferroelectric properties of strained tetragonal $\mathrm{PbZ}_{0.2} \mathrm{Ti}_{0.8} \mathrm{O}_{3}$ obtained on layerbylayer grown, defectfree singlecrystalline films, Advanced Materials 18, 1657 (2006).

[5] R. Guo, L. E. Cross, S.-E. Park, B. Noheda, D. E. Cox, and G. Shirane, Origin of the high piezoelectric response in $\mathrm{PbZr}_{1-x} \mathrm{Ti}_{x} \mathrm{O}_{3}$, Physical Review Letters 84, 5423 (2000).

[6] L. E. Cross, Relaxor ferroelectrics, Ferroelectrics 76, 241 (1987).

[7] S. Trolier-McKinstry and P. Muralt, Thin film piezoelectrics for MEMS, Journal of Electroceramics 12, 7 (2004).

[8] J. F. Scott, Applications of modern ferroelectrics, Science 315, 954 (2007).

[9] N. Izyumskaya, Y.-I. Alivov, S.-J. Cho, H. Morkoç, H. Lee, and Y.-S. Kang, Processing, structure, properties, and applications of PZT thin films, Critical Reviews in Solid State and Materials Sciences 32, 111 (2007).

[10] J. Son and Y.-H. Shin, Highly c-Oriented $\mathrm{PbZr}_{0.48} \mathrm{Ti}_{0.52} \mathrm{O}_{3}$ Thin Films on Glass Substrates, Electrochemical and Solid-State Letters 12, G20 (2009). 
[11] D. H. Kim, Y. K. Kim, S. Hong, Y. Kim, and S. Baik, Nanoscale bit formation in highly (111)-oriented ferroelectric thin films deposited on glass substrates for high-density storage media, Nanotechnology 22, 245705 (2011).

[12] S. S. Roy, H. Gleeson, C. P. Shaw, R. W. Whatmore, Z. Huang, Q. Zhang, and S. Dunn, Growth and characterisation of lead zirconate titanate (30/70) on indium tin oxide coated glass for oxide ferroelectric-liquid crystal display application, Integrated Ferroelectrics 29, 189 (2000).

[13] K. K. Uprety, L. E. Ocola, and O. Auciello, Growth and characterization of transparent $\mathrm{Pb}(\mathrm{Zi}, \mathrm{Ti}) \mathrm{O}_{3}$ capacitor on glass substrate, Journal of Applied Physics 102, 084107 (2007).

[14] E. Bruno, F. Ciuchi, M. Castriota, S. Marino, G. Nicastro, E. Cazzanelli, and N. Scaramuzza, Structural transformations of PZT 53/47 sol-gel films on different substrates driven by thermal treatments, Ferroelectrics 396, 49 (2010).

[15] M. Bayraktar, W. A. Wessels, C. J. Lee, F. A. van Goor, G. Koster, G. Rijnders, and F. Bijkerk, Active multilayer mirrors for reflectance tuning at extreme ultraviolet (EUV) wavelengths, Journal of Physics D: Applied Physics 45, 494001 (2012).

[16] R. H. T. Wilke, R. L. Johnson-Wilke, V. Cotroneo, W. N. Davis, P. B. Reid, D. A. Schwartz, and S. Trolier-McKinstry, Sputter deposition of PZT piezoelectric films on thin glass substrates for adjustable x-ray optics, Applied Optics 52, 3412 (2013).

[17] M. Bayraktar, A. Chopra, G. Rijnders, K. Boller, and F. Bijkerk, Wavefront correction in the extreme ultraviolet wavelength range using piezoelectric thin films, Optics Express 22, 30623 (2014).

[18] M. J. Haun, E. Furman, S. J. Jang, and L. E. Cross, Thermodynamic theory of the lead zirconate-titanate solid solution system, part V: Theoretical calculations, Ferroelectrics 99, 63 (1989).

[19] X.-h. Du, J. Zheng, U. Belegundu, and K. Uchino, Crystal orientation dependence of piezoelectric properties of lead zirconate titanate near the morphotropic phase boundary, Applied Physics Letters 72, 2421 (1998).

[20] A. Chopra, B. I. Birajdar, Y. Kim, M. Alexe, and D. Hesse, Enhanced ferroelectric and dielectric properties of (111)-oriented highly cation-ordered $\mathrm{PbSc}_{0.5} \mathrm{Ta}_{0.5} \mathrm{O}_{3}$ thin films, Journal of Applied Physics 114, 224109 (2013).

[21] V. Nagarajan, A. Roytburd, A. Stanishevsky, S. Prasertchoung, T. Zhao, L. Chen, J. Melngailis, O. Auciello, and R. Ramesh, Dynamics of ferroelastic domains in ferroelectric thin films, Nature Materials 2, 43 (2003).

[22] J. F. Scott and C. A. Paz de Araujo, Ferroelectric memories, Science 246, 1400 (1989). 
[23] J. Schwarzkopf and R. Fornari, Epitaxial growth of ferroelectric oxide films, Progress in Crystal Growth and Characterization of Materials 52, 159 (2006).

[24] K. Lefki and G. J. M. Dormans, Measurement of piezoelectric coefficients of ferroelectric thin films, Journal of Applied Physics 76, 1764 (1994).

[25] F. Xu, F. Chu, and S. Trolier-McKinstry, Longitudinal piezoelectric coeffcient measurement for bulk ceramics and thin films using pneumatic pressure rig, Journal of Applied Physics 86, 588 (1999).

[26] L. Chen, J. Li, J. Slutsker, J. Ouyang, and A. Roytburd, Contribution of substrate to converse piezoelectric response of constrained thin films, Journal of Materials Research 19, 2853 (2004).

[27] J. Ouyang, R. Ramesh, and A. L. Roytburd, Theoretical predictions for the intrinsic converse longitudinal piezoelectric constants of lead zirconate titanate epitaxial films, Advanced Engineering Materials 7, 229 (2005).

[28] Z. Wang, G. K. Lau, W. Zhu, and C. Chao, Influence of test capacitor features on piezoelectric and dielectric measurement of ferroelectric films, IEEE Transactions on Ultrasonics, Ferroelectrics, and Frequency Control 53, 15 (2006).

[29] K. Prume, P. Muralt, F. Calame, T. Schmitz-Kempen, and S. Tiedke, Piezoelectric thin films: evaluation of electrical and electromechanical characteristics for MEMS devices, IEEE Transactions on Ultrasonics, Ferroelectrics, and Frequency Control 54, 8 (2007).

[30] Z. Wang and J. Miao, Critical electrode size in measurement of $d_{33}$ coefficient of films via spatial distribution of piezoelectric displacement, Journal of Physics D: Applied Physics 41, 035306 (2008).

[31] S. Sivaramakrishnan, P. Mardilovich, A. Mason, A. Roelofs, T. SchmitzKempen, and S. Tiedke, Electrode size dependence of piezoelectric response of lead zirconate titanate thin films measured by double beam laser interferometry, Applied Physics Letters 103, 132904 (2013).

[32] A. L. Roytburd, S. P. Alpay, V. Nagarajan, C. S. Ganpule, and S. Aggarwal, Measurement of internal stresses via the polarization in epitaxial ferroelectric films, Physical Review Letters 85, 190 (2000).

[33] S. Bühlmann, B. Dwir, J. Baborowski, and P. Muralt, Size effect in mesoscopic epitaxial ferroelectric structures: Increase of piezoelectric response with decreasing feature size, Applied Physics Letters 80, 3195 (2002).

[34] V. Nagarajan, A. Stanishevsky, L. Chen, T. Zhao, B.-T. Liu, J. Melngailis, A. L. Roytburd, R. Ramesh, J. Finder, Z. Yu, R. Droopad, and K. Eisenbeiser, Realizing intrinsic piezoresponse in epitaxial submicron lead zirconate titanate capacitors on Si, Applied Physics Letters 81, 4215 (2002). 
[35] S. Zhang, H. Li, and M. Li, Size-dependent piezoelectric coefficient $d_{33}$ of $\mathrm{PbTiO}_{3}$ nanoparticles, Materials Letters 62, 2438 (2008).

[36] M. Bayraktar, A. Chopra, F. Bijkerk, and G. Rijnders, Nanosheet controlled epitaxial growth of $\mathrm{PbZ}_{0.52} \mathrm{Ti}_{0.48} \mathrm{O}_{3}$ thin films on glass substrates, Applied Physics Letters 105, 132904 (2014).

[37] R. E. Leuchtner and K. S. Grabowski, in Pulsed laser deposition of thin films, edited by D. B. Chrisey and G. K. Hubler (John Wiley \& Sons, Inc., New York, 1994), Chap. Ferroelectrics.

[38] L. Correra and S. Nicoletti, Large-area deposition of thin films by UV pulsed laser ablation, Materials Science and Engineering: B 32, 33 (1995).

[39] Z. Zhu, X. J. Zheng, and W. Li, Submonolayer growth of BaTiO 3 thin film via pulsed laser deposition: A kinetic Monte Carlo simulation, Journal of Applied Physics 106, 054105 (2009).

[40] M. Nijland, S. Kumar, R. Lubbers, D. H. A. Blank, G. Rijnders, G. Koster, and J. E. ten Elshof, Local control over nucleation of epitaxial thin films by seed layers of inorganic nanosheets, ACS Applied Materials \& Interfaces 6, 2777 (2014).

[41] T. Shibata, K. Fukuda, Y. Ebina, T. Kogure, and T. Sasaki, One-nanometerthick seed layer of unilamellar nanosheets promotes oriented growth of oxide crystal films, Advanced Materials 20, 231 (2008).

[42] J.-F. Li, K. Takagi, M. Ono, W. Pan, R. Watanabe, A. Almajid, and M. Taya, Fabrication and evaluation of porous piezoelectric ceramics and porositygraded piezoelectric actuators, Journal of the American Ceramic Society 86, 1094 (2003).

[43] S. N. Gwirc and C. A. Negreira, Characterization of porous thick film PZT composite for bilayer ultrasonic transducers, Ferroelectrics 321, 41 (2005).

[44] S. B. Lang and E. Ringgaard, Measurements of the thermal, dielectric, piezoelectric, pyroelectric and elastic properties of porous PZT samples, Applied Physics A 107, 631 (2012).

[45] Comsol Multiphysics.

[46] ULE ${ }^{\circledR}$ Corning Code 7972, Ultra Low Expansion Glass Datasheet, 2006.

[47] P. Verardi, M. Dinescu, F. Craciun, R. Dinu, and M. F. Ciobanu, Growth of oriented $\mathrm{Pb}\left(\mathrm{Zr}_{x} T i_{1-x}\right) \mathrm{O}_{3}$ thin films on glass substrates by pulsed laser deposition, Applied Physics A 69, S837 (1999). 


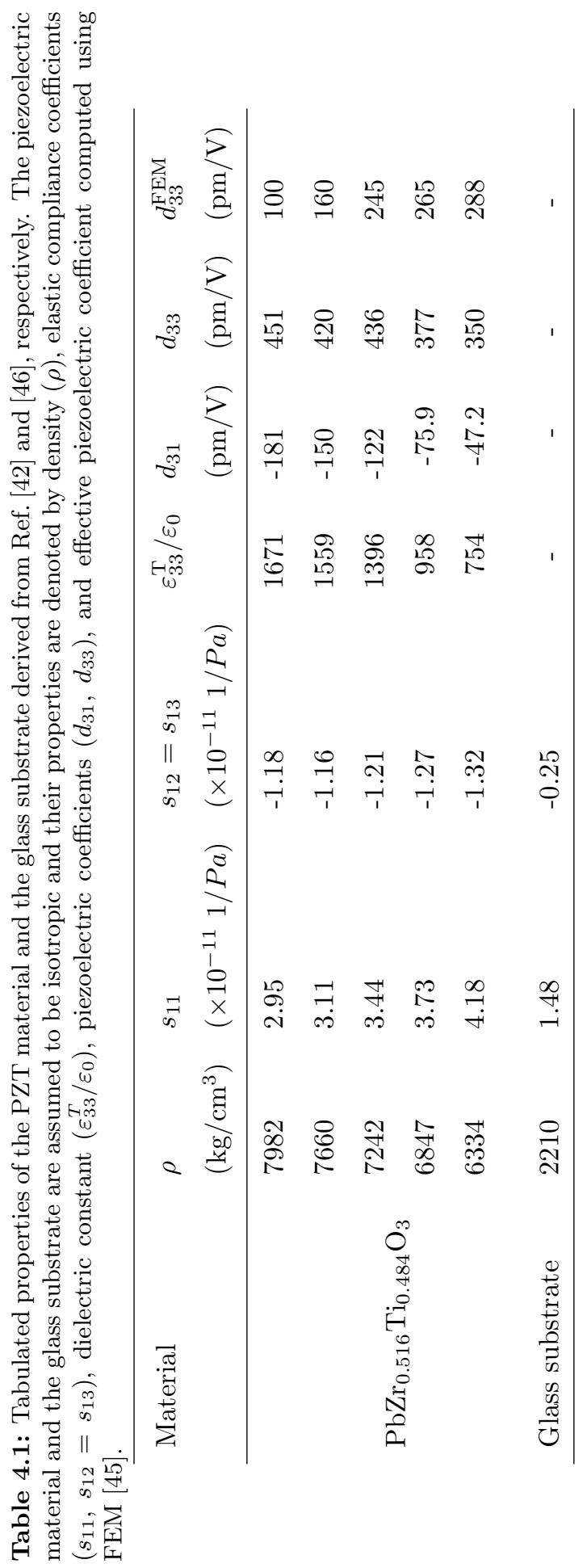





\title{
5 Wavefront correction in the extreme ultraviolet wavelength range using piezoelectric thin films
}

\begin{abstract}
A new scheme for wavefront correction in the extreme ultraviolet wavelength range is presented. The central feature of the scheme is the successful growth of crystalline piezoelectric thin films with the desired orientation on an amorphous glass substrate. The piezoelectric films show a high piezoelectric coefficient of $250 \mathrm{pm} / \mathrm{V}$.Using wavefront calculations we show that the grown films would enable high-quality wavefront correction, based on a stroke of $25 \mathrm{~nm}$, with voltages that are well below the electrical breakdown limit of the piezoelectric film.
\end{abstract}




\subsection{Introduction}

Highly reflective multilayer mirrors (MLM) are key optical components for the extreme ultraviolet (EUV) wavelength range [1, 2]. However, because all solid materials absorb strongly in the named wavelength range, EUV mirrors are easily subject to a significant heat load when high average powers are required. The associated temperature changes may impose severe distortions (aberrations) of the reflected wavefront which would reduce the optical performance. These distortions are generally non-uniform across the wavefront due to illumination conditions and may have magnitudes that are comparable to the operational wavelength [3-7]. To reach the intended optical performance it is essential to correct the wavefront distortions.

A technique that can perform this task is using adaptive optics based on deformable mirrors. In order to enable correction or shaping of the wavefront with fine resolution, the substrate of the deformable mirror has to be flexible, such as by using a thin membrane substrate with a reflective coating. An array of actuators at the backside adjustably deforms the membrane for removing the wavefront distortions in the reflected light [8-16]. At standard wavelengths, i.e., in the infrared or visible range, this approach has become a key solution to achieve diffraction limited resolution, e.g., in astronomy, vision or microscopy [17-20].

In the EUV range, where wavelengths are about two orders of magnitude shorter, the situation is quite different. Mechanical and thermal stability requirements scale with wavelength and easily reach into the sub-nanometer range. To provide such mechanical stability, the mirror substrates need to be chosen several centimeters thick. For achieving maximum thermal stability, amorphous glass materials with low coefficient of thermal expansion have to be chosen such as Fused Silica, Zerodur or Ultra Low Expansion (ULE) glass. However, choosing a substrate with high mechanical and thermal stability implies severe limitations for wavefront correction because the mirror surface cannot be deformed with fine resolution [21]. Recently, the residual expansion of thick Fused Silica glass substrates, driven by structured back-illumination with a heating light beam, has been utilized for wavefront correction [7]. This method indeed preserves the mechanical substrate stability but actuation becomes slow, about $0.1 \mathrm{~nm} / \mathrm{min}$ [7], and the transverse resolution of actuation at the mirror surface is limited to several centimeters by heat conduction. Deformable mirrors for grazing incidence EUV optics have been realized based on controlled bending of the substrate [2229]. But this method is especially hard to apply to the thick mirror substrates that are used in normal incidence EUV optics, hence a novel approach is desirable. Such approach has to be based on thick and thermally passive glass substrates to preserve mechanical and thermal stability. Nevertheless a sufficient large stroke of at least a quarter of an EUV wavelength (corresponding to $3.4 \mathrm{~nm}$ for the application example that will be considered here) has to be provided in combination with sufficiently fine resolution across the reflecting surface [7].

Here we present a piezoelectric thin film approach that provides these features of a high stability, a large stroke and a fine resolution. We demonstrate the basic working of the approach by fabricating single thin film actuator seg- 
ments, also called pixels $\left(200 \times 200 \mu \mathrm{m}^{2}\right.$ area, and about $2 \mu \mathrm{m}$ thick $)$ with which we demonstrate a large stroke of $25 \mathrm{~nm}$. The unique manner of achieving such large stroke is based on a crystalline piezoelectric film, lead zirconium titanate $\mathrm{PbZr}_{x} \mathrm{Ti}_{1-x} \mathrm{O}_{3}(\mathrm{PZT})$ at an $x=0.52$ composition, is deposited onto an amorphous glass substrate [30]. This choice of an amorphous glass substrate was made to show that our approach is compatible with the substrates normally used for EUV wavelengths that need to be made from amorphous glass. We note that, so far, all attempts to grow piezoelectric films on amorphous glass had resulted only in disordered polycrystalline films, which weakens or disables the piezoelectric response [27, 31-37].

The main breakthrough we report here is that we obtain a stroke of $25 \mathrm{~nm}$, which is well above the desired quarter-wavelength stroke of $3.4 \mathrm{~nm}$ by achieving, growth of crystalline piezoelectric films with desired (100)-orientation on amorphous glass substrate [30]. We note that the stroke of thin film piezoelectric actuators is not simply given by the intrinsic piezoelectric coefficient of the film material as if it were present in the bulk form. The reason is, as known from previous investigations of piezoelectric thin films [38-50], that the thin film is clamped by the underlying substrate. Qualitatively this leads to a reduction of the stroke if the transverse size of the thin film pixels is smaller than the thickness of the substrate. Predicting quantitative values needs a detailed modeling of the strain distribution. In the context of wavefront correction this raises the question whether a piezoelectric film of given pixel size still yields the required quarter-wavelength stroke and, at the same time, provides sufficient resolution for the corresponding application.

In the following, we first describe the deposition of piezoelectric thin film and its patterning into pixels. We determine the required pixel size for applications at $13.5 \mathrm{~nm}$ using the parameters in [7]. The required pixel size is calculated by expressing typical types of wavefront distortion as Zernike polynomials [51] and using the Marechal criterion for the quality of correction in terms of the Strehl ratio [17]. Finally we present measurements at single pixels to determine the absolute piezoelectric response and show that our approach easily fulfills the quantitative requirements for wavefront correction in the EUV wavelengths.

\subsection{Growth of piezoelectric films on glass substrates}

The stack of layers we deposited on a glass substrate is comprised of a buffer layer and a piezoelectric layer sandwiched between two electrode layers as schematically shown in Fig. 5.1(a). We note that PZT grows in polycrystalline form without preferred orientation if directly deposited on the amorphous substrate. In order to induce crystalline growth with the desired (100)-orientation we first cover the glass substrate with a $1.5 \mathrm{~nm}$ thick buffer layer consisting of crystalline nanosheets. Such nanosheets have been successfully applied earlier by Nijland et al. for growing films with improved magnetic properties [52], and we have followed their recipe [30]. Next, we have grown a $200 \mathrm{~nm}$ thick conductive epitaxial $\mathrm{LaNiO}_{3}$ (LNO) electrode layer. Besides serving as an electrode, 
the LNO layer also provides a crystalline template layer for crystalline growth of the piezoelectric layer $[33,34,53]$. In order to achieve a maximally strong piezoelectric response we selected $\mathrm{PZT}$ with $\mathrm{PbZr}_{0.52} \mathrm{Ti}_{0.48} \mathrm{O}_{3}$ composition. The piezoelectric coefficient is known to increase with thickness and saturate above $1500 \mathrm{~nm}$ [54]. A PZT layer of approximately $2000 \mathrm{~nm}$ thickness was grown on the LNO/Nanosheet/Glass structure. An LNO layer with $100 \mathrm{~nm}$ thickness was deposited as the top electrode. In order to increase the electrical conductivity and provide a homogeneous electric field across the top electrode, subsequently a $100 \mathrm{~nm}$ thick Pt layer was deposited on the LNO electrode. A typical crosssectional image of the layer stack recorded with a high resolution scanning electrode microscope (HR-SEM, Zeiss, MERLIN) is shown in Fig. 5.1(b). The SEM image shows well-defined columns as an indication of the successful crystalline growth. On top of the electrode we expect, in a next step, to deposit EUVreflective MLM layers (which are amorphous) without major problems, because it is relatively easy to deposit amorphous layers on crystalline structures [55]. Finally, using standard UV photolithography the deposited film structure was segmented into single quadratic pixels of $200 \times 200 \mu \mathrm{m}^{2}$ size as schematically shown in Fig. 5.1(a).
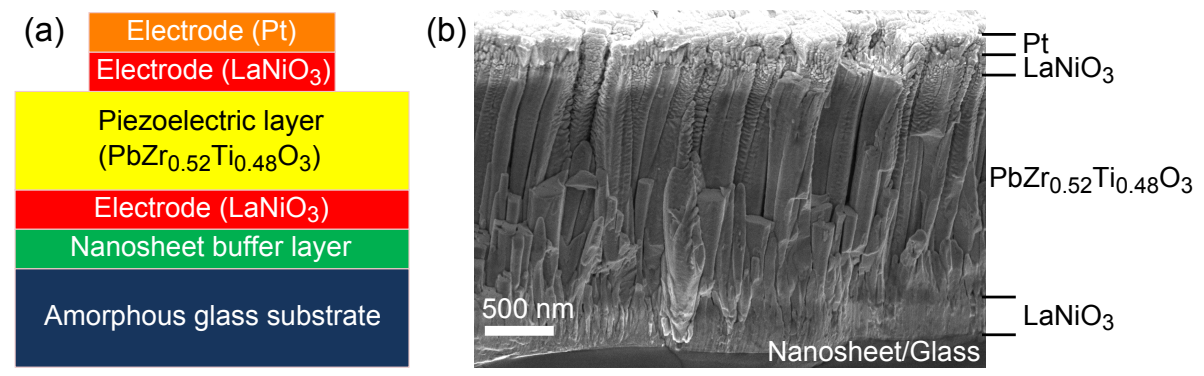

Figure 5.1: (a) Schematic view of the film stack structured into a pixel. (b) Crosssectional scanning electron microscope image of the crystalline piezoelectric actuator film stack deposited on a glass substrate.

\subsection{Calculation of suitable pixel size}

Providing crystalline piezoelectric films with (100)-orientation is known to maximize the piezoelectric response as compared to polycrystalline films [30]. But crystallinity is not the only parameter that affects the response. The piezoelectric response obtained with thin films in the direction of the surface normal (longitudinal response) depends also on the transverse, in-plane dimension of the deposited thin film pixel (named pixel size). The reason is that, upon expansion in the longitudinal dimension, the shrinking of the piezoelectric material is limited in the transverse dimension due to clamping to the underlying substrate, which reduces the effective piezoelectric response. The effect becomes more dominant with pixel sizes comparable to the substrate thickness, and a full 
quantification requires numerical modeling using the detailed mechanical parameters of the structure [38-50]. Clamping may be circumvented by choosing a pixel size that is either much smaller or much larger than the thickness of the substrate. However, small pixels increases the complexity of control and large pixels reduce the spatial resolution with which a wavefront can be corrected. This raises the question as to which size of pixels is required for wavefront correction and whether the stroke measured with a selected pixel size reaches the required value of quarter-wavelength.

In the following we calculate the pixel size that is required to correct a given wavefront distortion with sufficient quality. A standard way to express the strength and type of wavefront distortions is to expand them into Zernike polynomials, $Z_{n}$, which yields a set of Zernike coefficients, $a_{n}$. The magnitude of the Zernike coefficients indicate the strength of distortion, while the Zernike index, $n=1,2,3, \ldots$ where Zernike - Fringe ordering is used [51], indicates the degree of complexity of the wavefront distortion. First 36 Zernike polynomials and their shapes are shown in Appendix C. For definiteness and in view of its relevance for the considered EUV application at $\lambda_{E U V}=13.5 \mathrm{~nm}$, here we consider thermally induced wavefront distortions as expected for spatially non-uniform illumination [3-7]. For an exemplary mirror size of $20 \mathrm{~cm}$ diameter and $5 \mathrm{~cm}$ thickness it was found that the dominant orders of distortion are limited to about $n=20$, while higher orders can be neglected. The maximum strength of wavefront distortion found was half a wavelength, i.e., $\lambda_{E U V} / 2 \approx 6.8 \mathrm{~nm}$ [7]. The first four lowest-order coefficients (for tip, tilt and focusing) can be disregarded, i.e., $a_{n}=0$ for $n=1 \ldots 4$, because correction can be achieved without a deformable mirror, simply by alignment of the optical components. Figure 5.2(a) shows an example of the lowest order distortion that needs correction with a deformable mirror, $Z_{5}$, which is an astigmatic deformation, in this case of a wavefront with $20 \mathrm{~cm}$ diameter. Since the presented wavefront correction approach is to be obtained in reflection, the stroke needed for compensation of a distortion of half a wavelength is a quarter wavelength $\left(\lambda_{E U V} / 4 \approx 3.4 \mathrm{~nm}\right)$. Next we consider wavefront correction by a deformable mirror which comprises an array of thin film piezoelectric actuators with square shape and size $L$ (area $L^{2}$ ). To obtain the best correction, we vary the surface displacement of each pixel until the average wavefront distortion of the reflected light is minimized in each pixel. Figure 5.2(b) shows as an example the wavefront of Fig. 5.2(a) after correction for the case that a pixel size of $L=4 \mathrm{~cm}$ is chosen.

It can be seen that much of the astigmatism is removed in the chosen example. However, the quality of correction needs a quantification, such that it can be judged which pixel size is sufficient for the correction of Zernike distortions with orders of up to $n=20$. To provide such quantification of correction quality we first take the corrected wavefront and calculate its point spread function (PSF) using numerical wavefront propagation. The PSF is the normalized intensity distribution that is achieved in an image plane when propagating light from a distant point source, here, after first having undergone distortion and then being reflected by the deformable mirror. The obtained PSF is then compared with the maximum achievable quality of a perfectly diffraction-limited wavefront, for which 

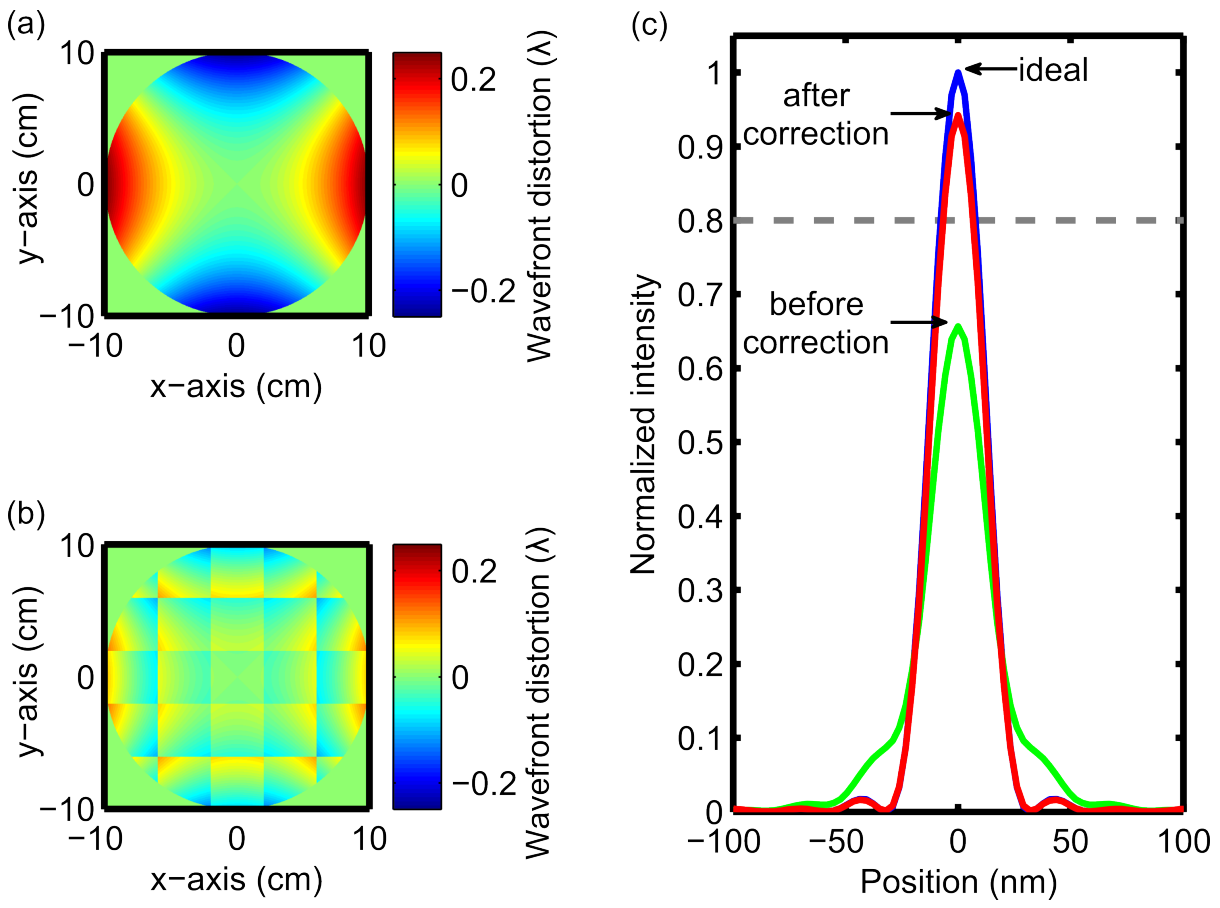

Figure 5.2: (a) Example of distorted wavefront, here, a $5^{\text {th }}$ order Zernike-polynomial $\left(Z_{5}\right.$, astigmatism) distortion with $a_{5}=\lambda_{E U V} / 2=6.8 \mathrm{~nm}$ amplitude and $20 \mathrm{~cm}$ diameter. (b) The same wavefront corrected by reflection at a deformable mirror comprising an array of $4 \times 4 \mathrm{~cm}^{2}$ square segments (pixels) made of thin film piezoelectric material. (c) Point spread function (PSF) calculated for the distorted wavefront (green, peak height $S=0.65$ ), after correction with the array of $4 \times 4 \mathrm{~cm}^{2}$ pixels (red, peak height $S=0.93$ ) and the ideally corrected, diffraction limited wavefront (blue line, $S=1$ ). The horizontal dashed gray line corresponds to a Strehl ratio of $S=0.8$ above which wavefront correction is generally considered as of high-quality.

we use the Strehl ratio, $S$, which is defined as the peak ratio of the two PSFs. Finally we apply the Marechal criterion $(S>0.8)$ in order to check whether the distortion can be considered as well-corrected, i.e., whether the wavefront correction is of high quality [17].

An example of such calculation is shown in Fig. 5.2(c), which compares the PSF of the astigmatically distorted wavefront of Fig. 5.2(a) (green curve) and the PSF of the corrected wavefront of Fig. 5.2(b) (red curve) to that of the ideal diffraction limited PSF (blue curve). We have normalized the latter, ideal PSF to have its peak value at unity, such that the horizontal dashed line corresponds to a Strehl ratio of $S=0.8$, above which the Marechal quality criterion is fulfilled. It can be seen that an array with 4 -cm-sized pixels is able to correct the incident low-quality wavefront $(S=0.65)$ with high quality $(S=0.93)$. 
As in the shown example for $Z_{5}$, we have calculated the quality of correction for the relevant higher order Zernike distortions, i.e., from the $Z_{6}$ to $Z_{35}$ using the same initial strength of distortion, $a_{n}=\lambda_{E U V} / 2$, in which we have stepwise varied the size of the square pixels, $L$. Figure 5.3 summarizes the results for $L=4 \mathrm{~cm}, 2 \mathrm{~cm}$ and $1 \mathrm{~cm}$ pixel sizes. It can be seen that the Strehl ratio increases with decreasing pixel size, i.e., with finer resolution of the correction, as expected. Starting from values as low as $S=0.4$ for $Z_{9}$ the Strehl ratio reaches the Marechal quality criterion (values above $S=0.8$ ) for all the Zernike polynomials up to $Z_{20}$, when a pixel size of $2 \mathrm{~cm}$ and smaller is used.

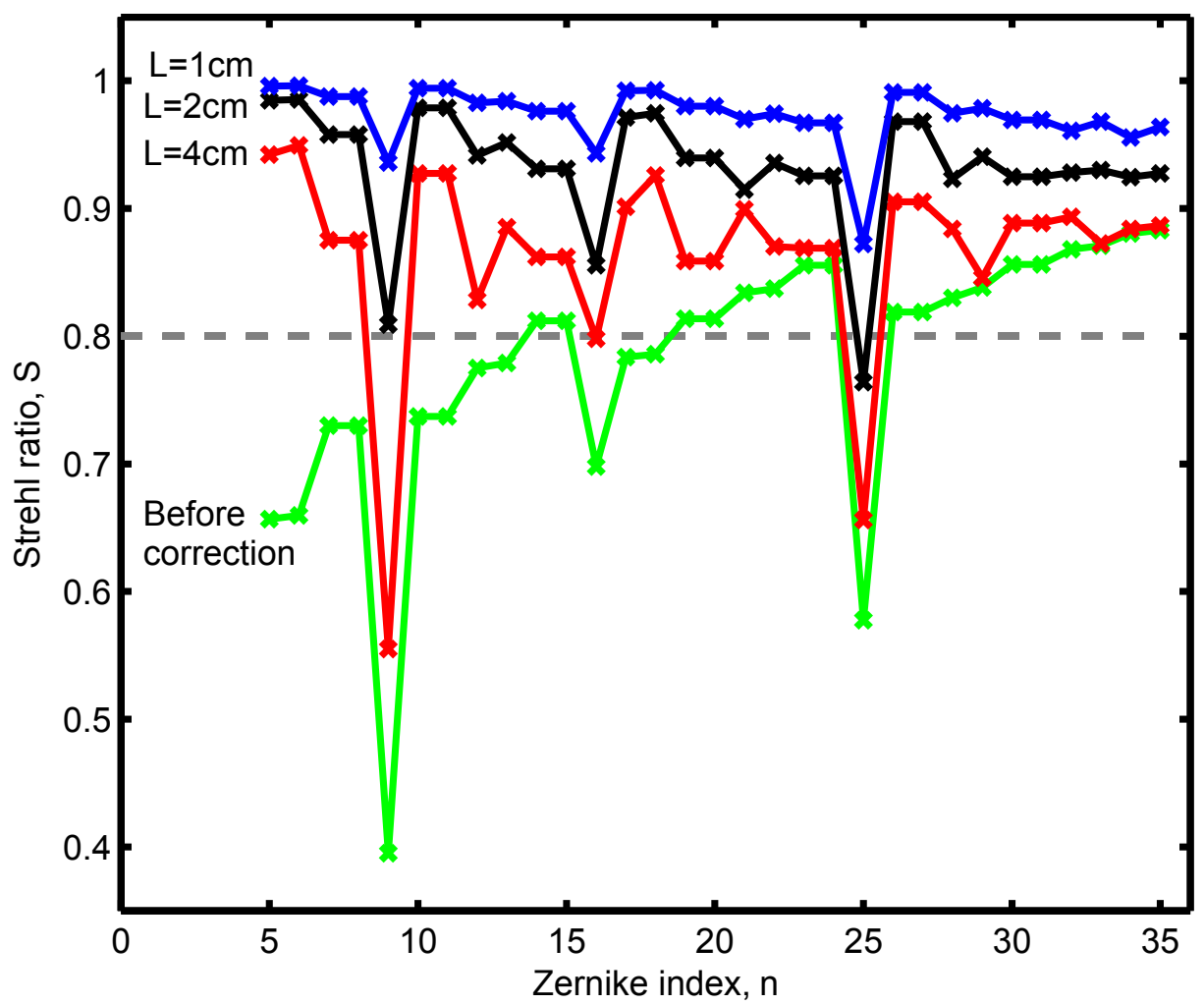

Figure 5.3: The Strehl ratio for aberrations in the shape of Zernike polynomials with a strength of $a_{n}=\lambda_{E U V} / 2=6.8 \mathrm{~nm}$ is shown for four different cases: before the correction and after the correction with a deformable mirror having a pixel size of $L=4 \mathrm{~cm}, 2 \mathrm{~cm}$ or $1 \mathrm{~cm}$. The Marechal criterion (Strehl ratio above $S=0.8$ ) that proves high correction quality is indicated with dashed gray line. 


\subsection{Measurement of piezoelectric response}

Having calculated the required size of the thin film piezoelectric pixels with which high-quality EUV wavefront correction is possible, the remaining step is to verify that the fabricated films are capable to provide a stroke of at least the required stroke of $3.4 \mathrm{~nm}$. The stroke is given by the product of the thin film piezoelectric coefficient in longitudinal direction $\left(d_{33, f}\right)$, which is also named as effective piezoelectric coefficient, and the maximum applicable voltage as limited by electrical breakthrough. As mentioned in Sec. 5.3, the effective piezoelectric coefficient in thin films depends on the pixel size due to clamping to the substrate. A closer look reveals that the absolute value of the effective piezoelectric coefficient does not depend on the absolute size of the pixel, $L$, but only on the ratio of the pixel size to the substrate thickness, $T$, i.e., on $\eta=L / T[42,48,50]$. We used finite element modeling [56] to find the value of $\eta$ where the clamping effect is strongest, i.e., at which value of $\eta$ the effective piezoelectric coefficient is smallest. This value corresponds to finding the worst case and guarantees that all other values for $\eta$ will provide at least as good or much better piezoelectric response. Our modeling revealed that the clamping is strongest and the piezoelectric coefficient reaches its minimum around a ratio of $\eta=0.4$ [48]. We have realized this ratio in our experiments by using samples with $L=200 \mu \mathrm{m}$ and $T=500 \mu \mathrm{m}$.

For measuring the longitudinal piezoelectric response of a pixel, an alternating voltage was applied at a frequency of $8 \mathrm{kHz}$. The surface displacement was recorded with a laser Doppler vibrometer (Polytec, MSA-400, with longitudinal resolution of $20 \mathrm{pm}$ at $8 \mathrm{kHz}$ ) [57]. The laser spot size used was approximately $5 \mu \mathrm{m}$ which defines the transverse spatial resolution. The spot was scanned across the pixel in order to determine the local surface displacement. An example of the typical measured piezoelectric response, expressed as piezoelectric coefficient, is shown in Fig. 5.4. It can be seen that the measured coefficient is very large, around $d_{33, f}=250 \mathrm{pm} / \mathrm{V}$, to our knowledge a record value for piezoelectric thin films on glass substrates. Typically, the piezoelectric coefficient shows a small variation with the temperature. Considering a realistic scenario in which the temperature varies by few degrees [7], the change in the piezoelectric coefficient is below $1 \%$ [58], which can be compensated in the industrial environments.

The voltage $V$ required to achieve a stroke of a quarter-wavelength, $\Delta T=$ $3.4 \mathrm{~nm}$, can be calculated by dividing the stroke by the measured piezoelectric coefficient. This calculation yields a value of $V=\Delta T / d_{33, f}=13.5 \mathrm{~V}$ for achieving the required wavefront correction.

It is important to note that this value of $13.5 \mathrm{~V}$ is, actually, a relatively small value which can be seen as follows. In order to determine the maximum possible stroke that can be achieved with our piezoelectric films, the maximum applicable voltage needs to be determined. We measured the maximum applicable voltage $\left(V_{\max }\right)$ and found that up to $100 \mathrm{~V}$ could be applied, repeatedly without any damage. At voltages higher than $100 \mathrm{~V}$ electrical breakdown occurred. This maximum voltage indicates that our film can achieve a high stroke of $\Delta T_{\max }=$ $V_{\max } \times d_{33, f}=25 \mathrm{~nm}$. With such high stroke, wavefront correction and shaping is certainly feasible for EUV application at $13.5 \mathrm{~nm}$ wavelength, and obviously 


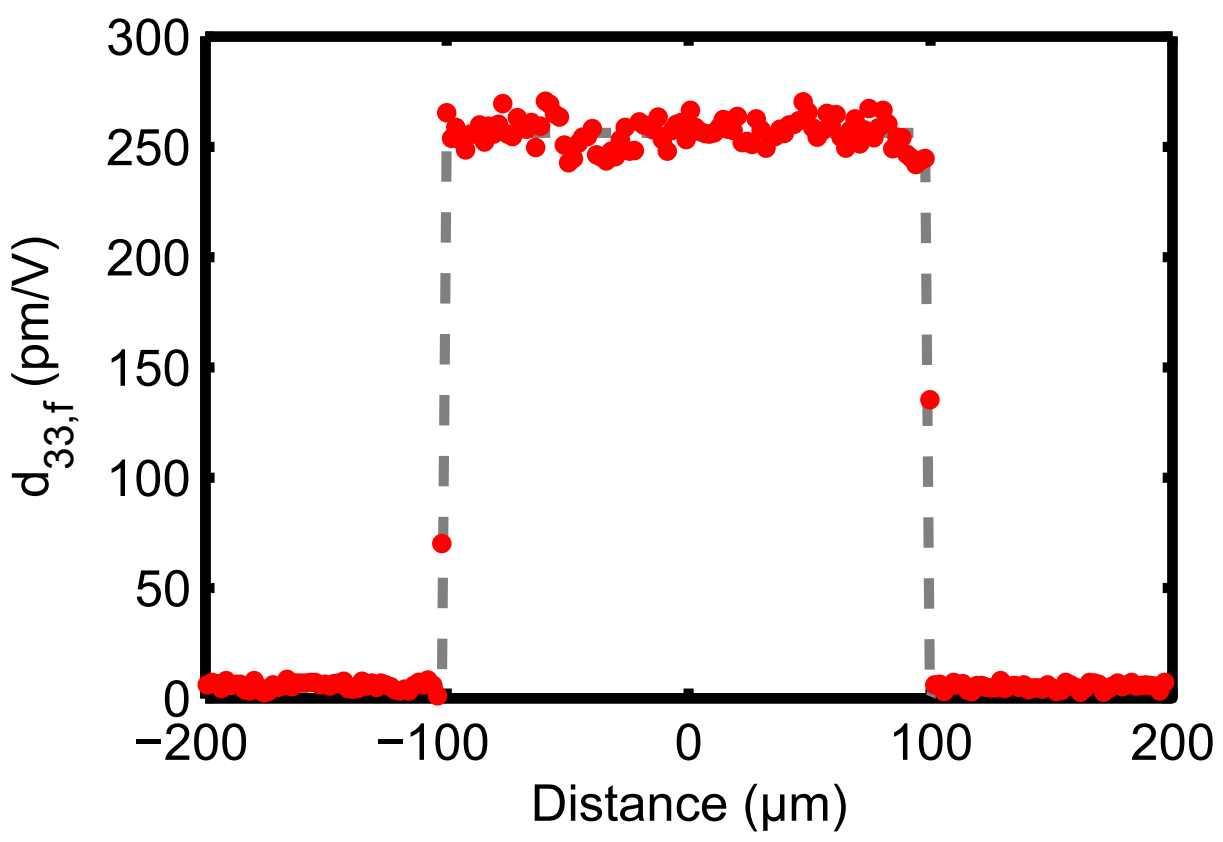

Figure 5.4: Measured effective longitudinal piezoelectric coefficient, $d_{33, f}$, of the PZT film for $200 \mu \mathrm{m}$ pixel size. Gray dashed line is shown as a guide to the eye.

even at longer wavelengths reaching $100 \mathrm{~nm}$.

\subsection{Summary and conclusion}

In summary, we have demonstrated the basic feasibility of a novel approach for wavefront correction in the EUV wavelength range based on crystalline piezoelectric thin films grown on amorphous glass substrates. The importance of this particular combination is that a sufficiently large stroke is provided by the film while a high mechanical and thermal stability can be provided by the thick and low thermal expansion glass substrates. Via expressing the typically expected wavefront distortions in terms of Zernike polynomials, we used wavefront calculations to determine the pixel size required for high-quality wavefront correction. The grown crystalline films showed a record-high piezoelectric coefficient, $d_{33, f}=250 \mathrm{pm} / \mathrm{V}$ measured at a pixel size that is relevant for EUV lithography. With such high piezoelectric coefficient, the quarter-wavelength stroke required for wavefront correction at a wavelength of $13.5 \mathrm{~nm}$ can be easily achieved with voltages that are much lower $(13.5 \mathrm{~V})$ than the measured voltage limit $(100 \mathrm{~V})$ given by electrical breakthrough. These results show that the presented wavefront correction approach using crystalline piezoelectric films on amorphous glass substrates is of high relevance for EUV wavefront correction and shaping. The 
approach can be of interest for other wavelengths and applications such as interferometry or astronomy with appropriate scaling.

\section{Acknowledgements}

This research program is funded by "Stichting Technologie en Wetenschap (STW)" under the contract 10448 with the project name "Smart Multilayer Interactive Optics for Lithography at Extreme UV wavelengths (SMILE)". The SMILE project is being sponsored by Carl Zeiss SMT GmbH. F. Bijkerk acknowledges the contributions from the FOM-Zeiss-ASML Industrial Partnership Programme CP3E. The authors would like to thank Prof. J. E. ten Elshof and Mr. Maarten Nijland for providing the nanosheets and Dr. Minh Nguyen for Pt coating.

\section{Bibliography}

[1] D. Attwood, Soft x-rays and extreme ultraviolet radiation: principles and applications (Cambridge University Press, 2007).

[2] EUV lithography, edited by V. Bakshi (SPIE Press, Bellingham, Washington, 2008).

[3] A. K. Ray-Chaudhuri, S. E. Gianoulakis, P. A. Spence, M. P. Kanouff, and C. D. Moen, Impact of thermal and structural effects on EUV lithographic performance, Proceedings of SPIE 3331, 124 (1998).

[4] Y. Li, K. Ota, and K. Murakami, Thermal and structural deformation and its impact on optical performance of projection optics for extreme ultraviolet lithography, Journal of Vacuum Science \& Technology B 21, 127 (2003).

[5] K. Liu, Y. Li, F. Zhang, and M. Fan, Transient thermal and structural deformation and its impact on optical performance of projection optics for extreme ultraviolet lithography, Japanese Journal of Applied Physics 46, 6568 (2007).

[6] G. Yang and Y. Li, Analysis and control of thermal and structural deformation of projection optics for 22-nm EUV lithography, Proceedings of SPIE 8322, 83222V (2012).

[7] R. Saathof, Adaptive optics to counteract thermal aberrations, Ph.D. thesis, Technische Universiteit Delft, The Netherlands, 2013.

[8] Boston Micromachines Corporation, 2014.

[9] Iris AO, Inc., 2014.

[10] ALPAO SAS, 2014.

[11] Flexible Optical B.V. (Okotech), 2014. 
[12] CILAS Corporate, 2014.

[13] AOA Xinetics, 2014.

[14] Microgate engineering, 2014.

[15] R. Hamelinck, R. Ellenbroek, N. Rosielle, M. Steinbuch, M. Verhaegen, and N. Doelman, Validation of a new adaptive deformable mirror concept, Proceedings of SPIE 7015, 70150Q (2008).

[16] R. Hamelinck, Adaptive deformable mirror: based on electromagnetic actuators, Ph.D. thesis, Technische Universiteit Eindhoven, The Netherlands, 2010 .

[17] J. W. Hardy, Adaptive optics for astronomical telescopes (Oxford University Press, New York, 1998).

[18] F. Roddier, Adaptive optics in astronomy (Cambridge University Press, Cambridge, 1999).

[19] Adaptive optics for vision science: Principles, practices, design and applications, edited by J. Porter, H. M. Queener, J. E. Lin, K. Thorn, and A. A. S. Awwal (John Wiley \& Sons, Inc, Hoboken, NJ, 2006).

[20] R. K. Tyson, Principles of adaptive optics, 3rd ed. (Taylor \& Francis Group, LLC, Boca Raton, Florida, 2011).

[21] S. Ravensbergen, P. Rosielle, and M. Steinbuch, Deformable mirrors with thermo-mechanical actuators for extreme ultraviolet lithography: Design, realization and validation, Precision Engineering 37, 353 (2013).

[22] P. Doel, C. Atkins, S. Thompson, D. Brooks, J. Yao, C. Feldman, R. Willingale, T. Button, D. Zhang, and A. James, Large thin adaptive x-ray mirrors, Proceedings of SPIE 6705, 67050M (2007).

[23] P. B. Reid, S. S. Murray, S. Trolier-McKinstry, M. Freeman, M. Juda, W. Podgorski, B. Ramsey, and D. Schwartz, Development of adjustable grazing incidence optics for Generation-x, Proceedings of SPIE 7011, 70110V (2008).

[24] W. N. Davis, P. B. Reid, and D. A. Schwartz, Finite element analyses of thin film active grazing incidence $x$-ray optics, Proceedings of SPIE 7803, 78030P (2010).

[25] D. Zhang, D. Rodriguez-Sanmartin, T. W. Button, C. Atkins, D. Brooks, P. Doel, C. Dunare, C. Feldman, A. James, A. Michette, W. Parkes, S. Pfauntsch, S. Sahraei, T. Stevenson, H. Wang, and R. Willingale, Development of piezoelectric actuators for active $x$-ray optics, Journal of Electroceramics 27, 1 (2011). 
[26] V. Cotroneo, W. N. Davis, V. Marquez, P. B. Reid, D. A. Schwartz, R. L. Johnson-Wilke, S. E. Trolier-McKinstry, and R. H. T. Wilke, Adjustable grazing incidence $x$-ray optics based on thin PZT films, Proceedings of SPIE 8503, 850309 (2012).

[27] R. H. T. Wilke, R. L. Johnson-Wilke, V. Cotroneo, W. N. Davis, P. B. Reid, D. A. Schwartz, and S. Trolier-McKinstry, Sputter deposition of PZT piezoelectric films on thin glass substrates for adjustable $x$-ray optics, Applied Optics 52, 3412 (2013).

[28] H. Mimura, S. Handa, T. Kimura, H. Yumoto, D. Yamakawa, H. Yokoyama, S. Matsuyama, K. Inagaki, K. Yamamura, Y. Sano, K. Tamasaku, M. Nishino, Y. Yabashi, T. Ishikawa, and K. Yamauchi, Breaking the $10 \mathrm{~nm}$ barrier in hard-x-ray focusing, Nature Physics 6, 122 (2010).

[29] S. Matsuyama, T. Kimura, H. Nakamori, S. Imai, Y. Sano, Y. Kohmura, K. Tamasaku, M. Yabashi, T. Ishikawa, and K. Yamauchi, Development of piezoelectric adaptive mirror for hard $x$-ray nanofocusing, Proceedings of SPIE 8503, 850303 (2012).

[30] M. Bayraktar, A. Chopra, F. Bijkerk, and G. Rijnders, Nanosheet controlled epitaxial growth of $\mathrm{PbZr}_{0.52} \mathrm{Ti}_{0.48} \mathrm{O}_{3}$ thin films on glass substrates, Applied Physics Letters 105, 132904 (2014).

[31] P. Verardi, M. Dinescu, F. Craciun, R. Dinu, and M. F. Ciobanu, Growth of oriented $\mathrm{Pb}\left(Z r_{x} T i_{1-x}\right) \mathrm{O}_{3}$ thin films on glass substrates by pulsed laser deposition, Applied Physics A 69, S837 (1999).

[32] K. K. Uprety, L. E. Ocola, and O. Auciello, Growth and characterization of transparent $\mathrm{Pb}(\mathrm{Zi}, \mathrm{Ti}) \mathrm{O}_{3}$ capacitor on glass substrate, Journal of Applied Physics 102, 084107 (2007).

[33] Y. Yu, M. Lai, and L. Lu, Highly (100) oriented $\mathrm{Pb}\left(\mathrm{Zr}_{0.52} \mathrm{Ti}_{0.48}\right) \mathrm{O}_{3} / \mathrm{LaNiO}_{3}$ films grown on amorphous substrates by pulsed laser deposition, Applied Physics A 88, 365 (2007).

[34] K. Kikuta, K. Noda, S. Okumura, T. Yamaguchi, and S.-i. Hirano, Orientation control of perovskite thin films on glass substrates by the application of a seed layer prepared from oxide nanosheets, Journal of Sol-Gel Science and Technology 42, 381 (2007).

[35] J. Son and Y.-H. Shin, Highly c-Oriented $\mathrm{PbZr}_{0.48} \mathrm{Ti}_{0.52} \mathrm{O}_{3}$ Thin Films on Glass Substrates, Electrochemical and Solid-State Letters 12, G20 (2009).

[36] R. H. T. Wilke, S. Trolier-McKinstry, P. B. Reid, and D. A. Schwartz, PZT piezoelectric films on glass for Gen-X imaging, Proceedings of SPIE 7803, 780300 (2010).

[37] D. H. Kim, Y. K. Kim, S. Hong, Y. Kim, and S. Baik, Nanoscale bit formation in highly (111)-oriented ferroelectric thin films deposited on glass substrates for high-density storage media, Nanotechnology 22, 245705 (2011). 
[38] K. Lefki and G. J. M. Dormans, Measurement of piezoelectric coefficients of ferroelectric thin films, Journal of Applied Physics 76, 1764 (1994).

[39] F. Xu, F. Chu, and S. Trolier-McKinstry, Longitudinal piezoelectric coefficient measurement for bulk ceramics and thin films using pneumatic pressure rig, Journal of Applied Physics 86, 588 (1999).

[40] A. Barzegar, D. Damjanovic, N. Ledermann, and P. Muralt, Piezoelectric response of thin films determined by charge integration technique: Substrate bending effects, Journal of Applied Physics 93, 4756 (2003).

[41] P. Gerber, A. Roelofs, C. Kügeler, U. Böttger, R. Waser, and K. Prume, Effects of the top-electrode size on the piezoelectric properties ( $d_{33}$ and $S$ ) of lead zirconate titanate thin films, Journal of Applied Physics 96, 2800 (2004).

[42] L. Chen, J. Li, J. Slutsker, J. Ouyang, and A. Roytburd, Contribution of substrate to converse piezoelectric response of constrained thin films, Journal of Materials Research 19, 2853 (2004).

[43] V. Nagarajan, Scaling of the piezoelectric response in ferroelectric nanostructures: An effective clamping stress model, Applied Physics Letters 87, 242905 (2005).

[44] Z. Wang, G. K. Lau, W. Zhu, and C. Chao, Influence of test capacitor features on piezoelectric and dielectric measurement of ferroelectric films, IEEE Transactions on Ultrasonics, Ferroelectrics, and Frequency Control 53, 15 (2006).

[45] K. Prume, P. Muralt, F. Calame, T. Schmitz-Kempen, and S. Tiedke, Piezoelectric thin films: evaluation of electrical and electromechanical characteristics for MEMS devices, IEEE Transactions on Ultrasonics, Ferroelectrics, and Frequency Control 54, 8 (2007).

[46] Z. Wang and J. Miao, Critical electrode size in measurement of $d_{33}$ coefficient of films via spatial distribution of piezoelectric displacement, Journal of Physics D: Applied Physics 41, 035306 (2008).

[47] N. Zalachas, B. Laskewitz, M. Kamlah, K. Prume, Y. Lapusta, and S. Tiedke, Effective piezoelectric coefficients of ferroelectric thin films on elastic substrates, 20, 683 (2009).

[48] G. J. T. Leighton and Z. Huang, Accurate measurement of the piezoelectric coefficient of thin films by eliminating the substrate bending effect using spatial scanning laser vibrometry, Smart Materials and Structures 19, 065011 (2010).

[49] X. Yan, W. Ren, H. Xin, P. Shi, X. Chen, and X. Wu, Influence of substrate deformation on piezoelectric displacement measurement of piezoelectric film, Ceramics International 39, Supplement 1, S583 (2013), the 8th Asian Meeting on Electroceramics (AMEC-8). 
[50] S. Sivaramakrishnan, P. Mardilovich, A. Mason, A. Roelofs, T. SchmitzKempen, and S. Tiedke, Electrode size dependence of piezoelectric response of lead zirconate titanate thin films measured by double beam laser interferometry, Applied Physics Letters 103, 132904 (2013).

[51] Zemax 13 Optical Design Program Users Manual, April 4, 2013.

[52] M. Nijland, S. Kumar, R. Lubbers, D. H. A. Blank, G. Rijnders, G. Koster, and J. E. ten Elshof, Local control over nucleation of epitaxial thin films by seed layers of inorganic nanosheets, ACS Applied Materials \& Interfaces 6, 2777 (2014).

[53] M.-S. Chen, T.-B. Wu, and J.-M. Wu, Effect of textured $\mathrm{LaNiO}_{3}$ electrode on the fatigue improvement of $\mathrm{Pb}\left(\mathrm{Zr}_{0.53} \mathrm{Ti}_{0.47}\right) \mathrm{O}_{3}$ thin films, Applied Physics Letters 68, 1430 (1996).

[54] D. M. Kim, C. B. Eom, V. Nagarajan, J. Ouyang, R. Ramesh, V. Vaithyanathan, and D. G. Schlom, Thickness dependence of structural and piezoelectric properties of epitaxial $\mathrm{Pb}\left(\mathrm{Zr}_{0.48} \mathrm{Ti}_{0.52}\right) \mathrm{O}_{3}$ films on Si and $\mathrm{SrTiO}_{3}$ substrates, Applied Physics Letters 88, 142904 (2006).

[55] E. Louis, A. E. Yakshin, T. Tsarfati, and F. Bijkerk, Nanometer interface and materials control for multilayer EUV-optical applications, Progress in Surface Science 86, 255 (2011).

[56] Comsol Multiphysics.

[57] Polytec MSA-400 Micro System Analyzer User Manual, 2005.

[58] Z. Q. Zhuang, J. Haun, M.J. Sei-Joo, and L. E. Cross, Composition and temperature dependence of the dielectric, piezoelectric and elastic properties of pure PZT ceramics, IEEE Transactions on Ultrasonics, Ferroelectrics, and Frequency Control 36, 413 (1989). 


\section{Active multilayer mirrors for reflectance tuning at extreme ultraviolet (EUV) wavelengths}

We propose an active multilayer mirror structure for extreme ultraviolet (EUV) wavelengths, which can be adjusted to compensate for reflectance changes. The multilayer structure tunes the reflectance via an integrated piezoelectric layer that can change its dimension due to an externally applied voltage. Here, we present design and optimization of the mirror structure for maximum reflectance tuning. In addition, we present preliminary results showing that the deposition of piezoelectric thin films with the requisite layer smoothness and crystal structure is possible. Finally piezoelectric coefficient measurement $\left(\mathrm{d}_{33}=60 \mathrm{pm} / \mathrm{V}\right)$ of the film is presented. 


\subsection{Introduction}

Multilayer mirrors (MLM) are commonly used at extreme ultraviolet (EUV) and $\mathrm{x}$-ray wavelengths because their high reflectance near normal incidence operation allows high numerical aperture imaging systems. MLMs find applications in astronomy [1, 2], microscopy [3, 4], spectroscopy [5], lasers [6], and next generation EUV lithography (EUVL) systems [7], the latter being the main driving force of the field. MLMs for EUVL systems are fabricated by depositing many pairs of Mo and Si layers such that the Bragg condition is satisfied at normal incidence for a wavelength of $13.5 \mathrm{~nm}$ [8]. Maximum reflectance is achieved, typically, when the period of the Mo/Si bilayer is $6.9 \mathrm{~nm}$ and the thickness of the Mo is $40 \%$ of the bilayer period. For such thin films, roughness, diffusion, compound formation in the $\mathrm{Mo} / \mathrm{Si}$ interface and crystallization are the dominant factors affecting the final reflectance of the mirror [9-12].

The reflectance of Mo/Si mirrors are typically $69.5 \%$, which can be increased up to $70.3 \%$ by integrating diffusion barriers and reflectivity enhancing layers into the MLM stack [13, 14]. However, even at its best, $30 \%$ of the incoming light is absorbed by the mirror, creating significant heat load, which may, under extreme conditions, cause some changes of the bilayer period and degradation of the reflectance with time. Since the mirror is not usually evenly illuminated, the mirror performance does not degrade uniformly. In this article, we present the design and preliminary experimental results for a new active MLM structure. An integrated piezoelectric layer that can change its dimension via external voltage separates two MLMs. The interference between the waves reflected from the bottom and top stack can, thereby, be adjusted to increase or reduce the reflectance. Small independent regions can be patterned while deposition of the active MLM on large substrates to make local reflectance adjustments. In section 2, details of the active MLM and the simulation of its reflectance using the IMD software package are explained [15]. In section 3, the reflectance tuning range, the effect of active layer thickness and the absorption are explained. In section 4 , the first fabrication results of the active piezoelectric films using pulsed laser deposition (PLD) are presented.

\subsection{Active multilayer mirror structure}

The active MLM structure was designed by integrating an active layer between two Mo/Si MLMs, as shown in Fig. 6.1(a). The structure is defined from bottom to top as a) base Mo/Si MLM stack, b) buffer layer, c) electrode/piezoelectric layer/electrode heterostructure and d) the top Mo/Si MLM stack. The top layer of the base Mo/Si MLM stack is amorphous; therefore, a buffer layer is needed to provide a template for the deposition of the crystalline heterostructure [16, 17]. The buffer layer and the heterostructure are deposited at elevated temperatures $\left(600{ }^{\circ} \mathrm{C}\right)$ to restrict the growth of the amorphous phase. Therefore, more chemically stable MLMs with diffusion barriers replace the standard Mo/Si MLMs as the base MLM stack [18-21]. In the simulation, $\mathrm{SrTiO}_{3}(\mathrm{STO}), \mathrm{SrRuO}_{3}(\mathrm{SRO})$, and $\mathrm{PbZr}_{0.20} \mathrm{Ti}_{0.80} \mathrm{O}_{3}(\mathrm{PZT})$ were used as the buffer, electrodes, and piezoelec- 
tric layers, respectively. The layer thicknesses are limited to integer multiples of the out-of-plane lattice parameters, which are $3.91 \AA, 3.95 \AA$ and $4.19 \AA$ for STO, $\mathrm{SRO}$, and PZT, respectively. The thickness of the buffer layer and the electrodes are taken as 50 unit cells, which corresponds to $19.55 \mathrm{~nm}$ and $19.75 \mathrm{~nm}$ respectively as listed in Table 6.1. In Fig. 6.1(b), the reflectance of the active mirror with respect to the piezoelectric layer thickness is plotted for monochromatic $13.5 \mathrm{~nm}$ light at normal incidence for 24 and 55 bilayers at the top and the base MLMs respectively. The reflectance and the absorption of the top and base MLM stacks are listed in Table 6.1. The reflectance curve is described by the principles of a lossy Fabry-Perot interferometer. When the active mirror is illuminated from the top, light waves are partially absorbed and partially reflected from the top and the base MLMs in which separation between them determines the phase difference between the reflected waves and, therefore, the total reflectance of the mirror for monochromatic light. In this design, the contrast of the interference between reflected waves, shown in Fig. 6.1(b), decreases as the piezoelectric layer thickness increases, due to the increasing absorption of the piezoelectric layer.
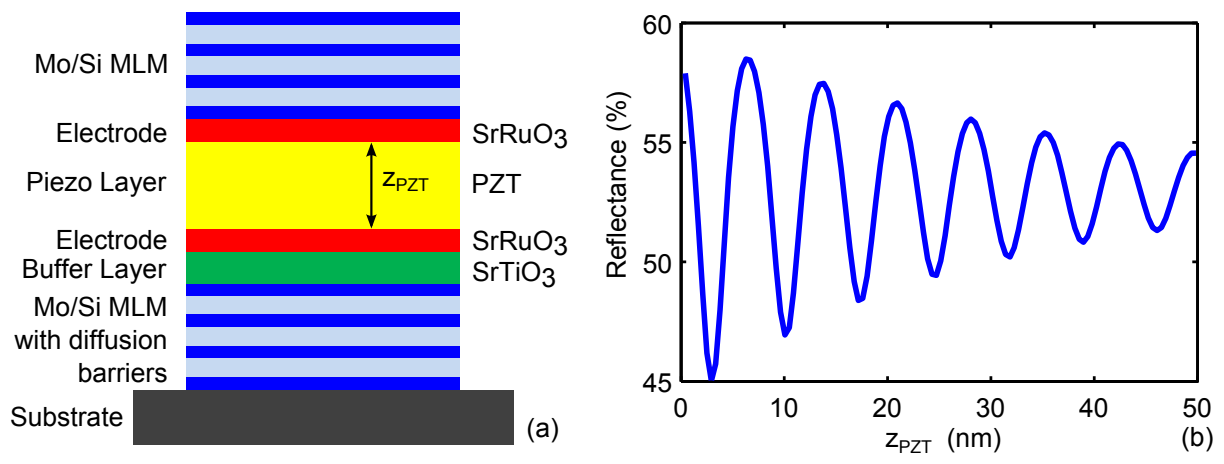

Figure 6.1: (a) The active multilayer mirror (MLM) structure was designed by placing an active piezoelectric layer, along with the necessary electrodes and buffer layer, between two Mo/Si MLMs. (b) Reflectance of the active MLM as a function of piezoelectric layer thickness.

\subsection{Reflectance tuning range}

In order to explain the reflectance tuning in more detail, a reflectance value for the relaxed structure is chosen to be $R_{r}=50 \%$ corresponding to a layer thickness around $z_{r}=z_{P Z T}=25 \mathrm{~nm}$ as shown in Fig. 6.2(a) (As can be seen in Fig. 6.1(b), $R_{r}=50 \%$ occurs for multiple layer thickness values; finding the optimum layer thickness is explained at the end of this section). Denoting the maximum strain of the piezoelectric layer as $\varepsilon$, the thickness of the layer can be increased up to $z_{e}=(1+\varepsilon) z_{r}$ and the reflectance value at this layer thickness will be the reflectance value of the extended mirror $R_{e}$. For the parameters given 
Table 6.1: Parameters used in the simulation. Bulk density and out-of-plane lattice constant values are taken from ICDD database [22].

\begin{tabular}{|c|c|c|c|c|c|}
\hline \multirow[t]{2}{*}{ Parameter } & \multirow[t]{2}{*}{$\mathrm{SrTiO}_{3}$} & \multirow[t]{2}{*}{$\mathrm{SrRuO}_{3}$} & \multirow[t]{2}{*}{$\mathrm{PbZr}_{0.20} \mathrm{Ti}_{0.80} \mathrm{O}_{3}$} & \multirow{2}{*}{$\begin{array}{l}\text { Top } \\
\text { MLM }\end{array}$} & \multirow{2}{*}{$\begin{array}{l}\text { Base } \\
\text { MLM }\end{array}$} \\
\hline & & & & & \\
\hline Density $\left(\mathrm{g} / \mathrm{cm}^{3}\right)$ & 5.117 & 7.058 & 7.376 & & \\
\hline $\begin{array}{l}\text { Out-of-plane lattice } \\
\text { constant }(\AA)\end{array}$ & 3.91 & 3.95 & 4.19 & & \\
\hline Thickness (nm) & 19.55 & 19.75 & variable & & \\
\hline Number of bilayers & & & & 24 & 55 \\
\hline Reflectance(\%) & & & & 40.4 & 51.3 \\
\hline Absorption (\%) & & & & 25.4 & 33.7 \\
\hline
\end{tabular}

in Table 6.1 , a reflectance tuning close to $\Delta R=4 \%$ is achieved for a maximum strain of $\varepsilon=5 \%$. The change in the reflectance with respect to piezoelectric layer thickness is shown Fig. 6.2(a). Here, $\varepsilon=5 \%$ is taken to clearly show the reflectance tuning, but, reflectance tuning for more realistic $\varepsilon$ values will be discussed at the end of this section.

Although it is instructive to explain the reflectance tuning process using monochromatic sources, broadband light sources are also used in optical systems. Assuming a light source with a power spectrum that is uniform in the range of $12.5 \mathrm{~nm}$ and $14.5 \mathrm{~nm}$, the reflectance spectrum of the mirror in its extended and relaxed states are shown in Fig. 6.2(b). The change in the integrated reflectance between the extended and relaxed states is relatively small. On the other hand, assuming an optical system with ten mirrors, in which for instance only one of the mirrors is active, a significant reflectance tuning can be obtained because of the narrow spectrum caused by the reflection from the nine mirrors. The normalized reflectance spectrum after nine mirrors, $R_{9 m}$, is shown in Fig. 6.2(b). In order to see the effect of the broadband source on the reflectance tuning range and to make it comparable with the reflectance tuning range for the monochromatic source, integrated reflectance must be normalized. Here we define normalized integrated reflectance as:

$$
\langle\text { Reflectance }\rangle=\frac{\int_{\lambda} R\left(\lambda, z_{P Z T}\right) \mathrm{d} \lambda}{\max \left\{\int_{\lambda} R\left(\lambda, z_{P Z T}\right) \mathrm{d} \lambda\right\}} \times \max \left\{R\left(\lambda_{0}, z_{P Z T}\right)\right\},
$$

where $\lambda_{0}=13.5 \mathrm{~nm}$. The normalized integrated reflectance is plotted in Fig. 6.2(c). The normalized integrated reflectance tuning range for $\varepsilon=5 \%$ is close to $\langle\Delta R\rangle=2 \%$, which is lower than the reflectance tuning range for a monochromatic source. 

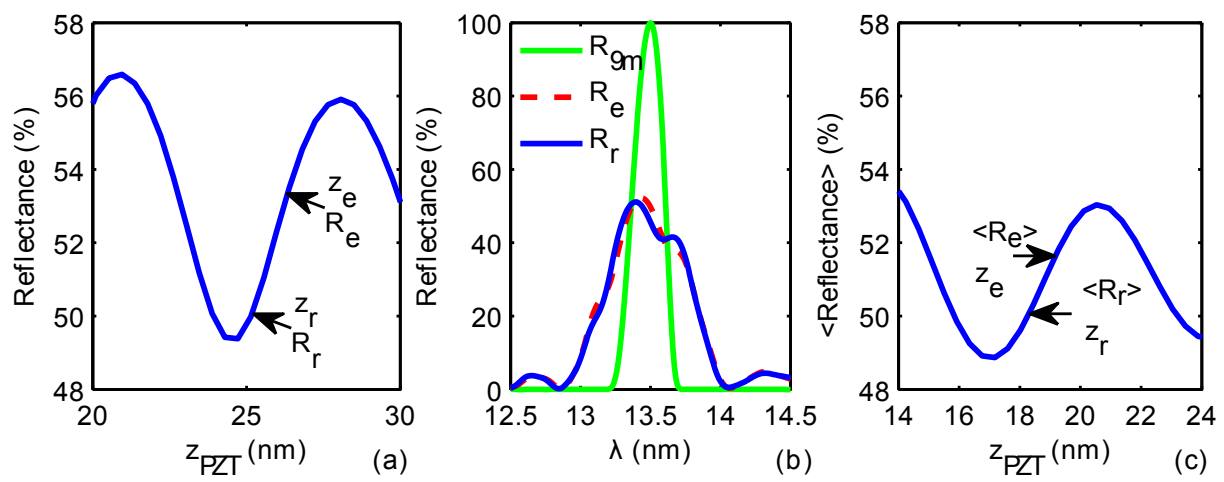

Figure 6.2: (a) Reflectance tuning for a monochromatic source. Reflectance of the active mirror can be increased from $R_{r}$ to $R_{e}$ by applying a voltage and increasing the thickness of the piezoelectric layer from $z_{r}$ to $z_{e}$. (b) Reflectance curves of an active mirror at its relaxed $\left(R_{r}\right)$ and extended state $\left(R_{e}\right)$ and the reflectance curve of nine Mo/Si MLMs $\left(R_{9 m}\right)$ when they are illuminated with a broadband source having a power spectrum that has uniform distribution in the range of $12.5 \mathrm{~nm}$ and $14.5 \mathrm{~nm}$. The tuning range for the integrated reflectance for a single active mirror is minor, but, when the number of MLMs in the optical system increases, the cascaded mirrors have a filtering effect and only central part of the spectrum around $13.5 \mathrm{~nm}$ is reflected, significantly improving the tuning range. (c) Normalized integrated reflectance of the active mirror can be increased from $\langle R\rangle_{r}$ to $\langle R\rangle_{e}$ by applying a voltage.

Up to now, the reflectance tuning has been calculated for the layer thickness parameters given in Table 6.1, but, as can be seen from Fig. 6.1(b), the relaxed reflectance value $\left(R_{r}\right)$, can be satisfied for multiple piezoelectric layer thicknesses. Limiting ourselves to regions with a positive change in reflectance with respect to increasing thickness, the maximum reflectance tuning has been calculated for multiple piezoelectric layer thicknesses. In Fig. 6.3, optimum reflectance tuning ranges $(\Delta R)$, for different piezoelectric layer thicknesses $\left(z_{P Z T}\right)$ and different maximum piezoelectric responses $(\varepsilon)$ are plotted for monochromatic and broadband sources where the piezoelectric layer thicknesses corresponding to $R_{r}$ are shown with markers. For both sources, when the piezoelectric layer thickness is below $15 \mathrm{~nm}$, the dimension change of the piezoelectric layer is not sufficient to cause enough reflectance tuning. On the other hand, when the piezoelectric layer thickness exceeds $30 \mathrm{~nm}$, then the absorption in the piezoelectric layer becomes dominant and the reflectance tuning range decreases. For both sources, the maximum reflectance tuning ranges are obtained for layer thicknesses around $20 \mathrm{~nm}$. 
M onochromatic Source $(\lambda=13.5 \mathrm{~nm})$

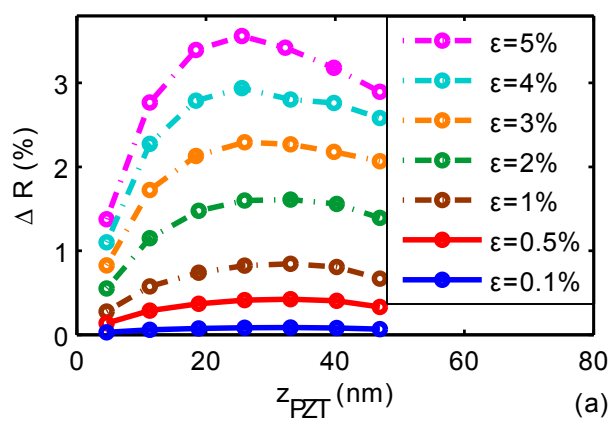

Broadband Source

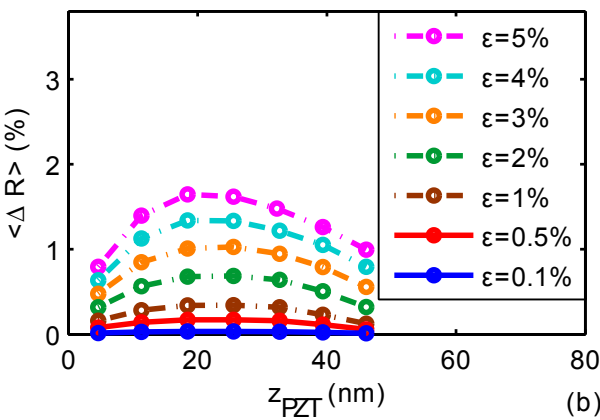

Figure 6.3: Optimum reflectance tuning ranges for (a) monochromatic, and (b) broadband light sources, for different maximum piezoelectric responses $\varepsilon$. For each rising region of a reflectance peak (see Fig. 6.1(b)), a different reflectance tuning range was calculated, which are shown by markers. Maximum reflectance tuning ranges are obtained for layer thicknesses around $20 \mathrm{~nm}$.

\subsection{Experimental results}

In fabricating the active MLM structure, the roughness and the maximum piezoelectric response of the active layer are two important parameters for the maximum reflectance and the reflectance tuning range. We used pulsed laser deposition (PLD), which is often preferred for its capability of growing high quality complex piezoelectric oxides, to grow electrode/piezoelectric film/electrode heterostructures to investigate their roughness and piezoelectric response [23]. As with our calculations, we use layer thicknesses that are expected to be relevant to real-world applications. We deposited epitaxial heterostructures consisting of $20 \mathrm{~nm}$ SRO, $30 \mathrm{~nm}$ PZT and $20 \mathrm{~nm}$ SRO on STO substrates with (001) orientation. The STO substrate was chosen because it is one of the candidate buffer layers for the active MLM design, due to its low lattice mismatch with the electrodes and the piezoelectric layer materials. The low lattice mismatch minimizes the in-plane stress and therefore results in optimized smooth growth.

The STO substrate was treated with $\mathrm{BHF}$ solution to obtain a $\mathrm{TiO}_{2}$ termination layer, followed by annealing at $950^{\circ} \mathrm{C}$ for 1.5 hour before deposition. A $\mathrm{KrF}$ excimer laser (wavelength $248 \mathrm{~nm}$ ) was used to ablate the target materials with laser fluences of $2.1 \mathrm{~J} / \mathrm{cm}^{2}$ at a $1 \mathrm{~Hz}$ repetition rate and $2.5 \mathrm{~J} / \mathrm{cm}^{2}$ at $10 \mathrm{~Hz}$ repetition rate for SRO and PZT, respectively. During the deposition, the substrate temperature was kept at $600{ }^{\circ} \mathrm{C}$ and the distance to the target was $50 \mathrm{~mm}$. A background pressure of 0.6 mbar was used during SRO deposition with gas ratios of $50 \% \mathrm{Ar}$ and $50 \% \mathrm{O}_{2}$, which resulted in atomically smooth growth [24]. PZT was deposited at five different $\mathrm{O}_{2}$ partial pressures to find the optimum $\mathrm{O}_{2}$ pressure to grow a smooth PZT film. The surface roughness of the substrates and the heterostructures were measured using a Nanoscope IV (Veeco Instruments, NY, USA) atomic force microscope in tapping mode ex-situ. The 
measured root mean square (RMS) surface roughness values as a function of $\mathrm{O}_{2}$ partial pressure are plotted in Fig. 6.4(a). It can be seen that an RMS surface roughness of $0.3 \mathrm{~nm}$ was obtained for 0.175 mbar $\mathrm{O}_{2}$ partial pressure, which is close to the RMS surface roughness of the substrate $(0.2 \mathrm{~nm})$. Layer thicknesses were determined by reflectivity measurements and the crystal structure by x-ray diffraction using a Bruker d8 Discover diffractometer (Bruker, Karlsruhe, Germany) (Results are not shown). The $\theta-2 \theta$ scans show that the PZT has (001) orientation for the smoothest heterostructures. The local piezoelectric response of a second heterostructure (SRO/PZT), deposited under identical conditions is shown in Fig. 6.4(b). The piezoelectric coefficient $\left(d_{33}\right)$ and the deformation was recorded on the local ferroelectric domain structure using a Nanoscope III (Veeco Instruments, NY, USA) piezoelectric response force microscope. As it can be seen from the Fig. 6.4(b), a $d_{33}$ value of $60 \mathrm{pm} / \mathrm{V}$ was measured.
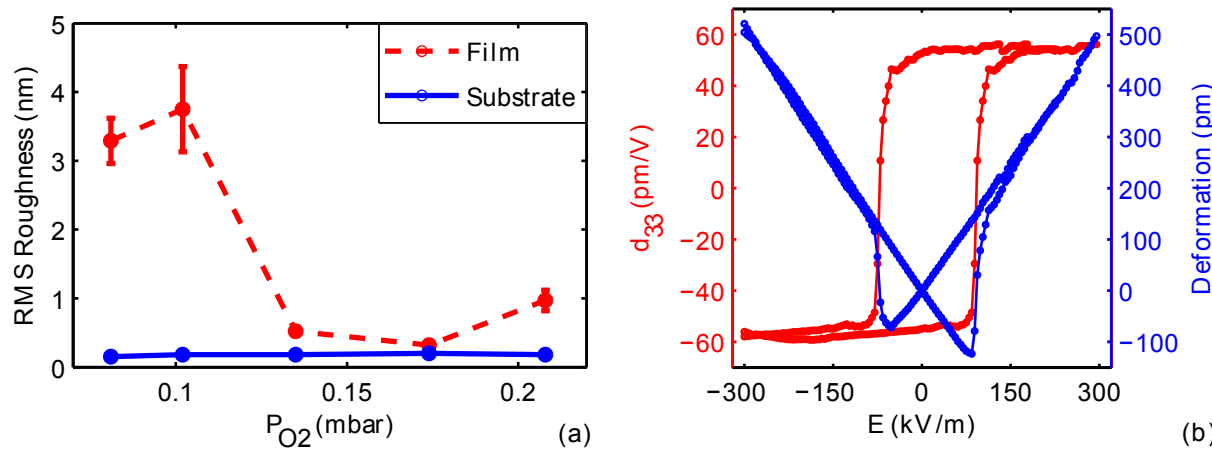

Figure 6.4: (a) RMS roughness of the STO substrate and the SRO/PZT/SRO heterostructure. (b) The piezoelectric coefficient and the deformation measurement for $30 \mathrm{~nm}$ thick PZT thin film deposited at 0.175 mbar $\mathrm{O}_{2}$ partial pressure.

\subsection{Conclusions}

In conclusion, the design of an active multilayer EUV mirror (MLM) structure has been presented. The active MLM was designed by integrating a piezoelectric layer (with electrodes and buffer layer) between two Mo/Si MLMs to allow reflectance tuning by applying an external voltage. Conditions necessary to optimize the piezoelectric layer thickness for maximum reflectance tuning were calculated. Piezoelectric films with $30 \mathrm{~nm}$ thickness have been deposited at different $\mathrm{O}_{2}$ pressures in order to investigate the RMS surface roughness and the piezoelectric response. RMS roughness of the heterostructure can be decreased down to $0.3 \mathrm{~nm}$, which is comparable to the RMS substrate roughness of $0.2 \mathrm{~nm}$. The piezoelectric coefficient of the film is measured to be $d_{33}=60 \mathrm{pm} / \mathrm{V}$. 


\section{Acknowledgements}

This research program is funded by the Technology Foundation (STW) under contract 10448 "Smart Multilayer Interactive Optics for Lithography at Extreme UV wavelengths (SMILE)". This work is additionally supported by the "FOM Industrial Partnership Programme (CP3E)" which is carried out under contract with Carl Zeiss SMT GmbH, Oberkochen, ASML, Veldhoven, and the "Stichting voor Fundamenteel Onderzoek der Materie (FOM)", the latter being financially supported by the "Nederlandse Organisatie voor Wetenschappelijk Onderzoek (NWO)".

\section{Bibliography}

[1] L. Golub, M. Herant, K. Kalata, I. Lovas, G. Nystrom, F. Pardo, E. Spiller, and J. Wilczynski, Sub-arcsecond observations of the solar X-ray corona, Nature 344, 842 (1990).

[2] D. S. Martínez-Galarce, A. B. C. Walker, II, D. B. Gore, C. C. Kankelborg, R. B. Hoover, T. W. Barbee, Jr., and P. F. X. Boerner, High resolution imaging with multilayer telescopes: resolution performance of the MSSTA II telescopes, Optical Engineering 39, 1063 (2000).

[3] I. Artioukov, V. Asadchikov, Y. S. Kas'yanov, A. Fedorenko, V. Kondratenko, R. Serov, A. Vinogradov, and S. Yulin, Schwarzschild soft-x-ray microscope for imaging of nonradiating objects, Optics Letters 20, 2451 (1995).

[4] A. Sakdinawat and D. Attwood, Nanoscale X-ray imaging, Nature Photonics 4, 840 (2010).

[5] W. Moos, A. Zwicker, S. Regan, and M. Finkenthal, Layered synthetic microstructures for soft $x$-ray spectroscopy of magnetically confined plasmas, Review of Scientific Instruments 61, 2733 (1990).

[6] A. M. Hawryluk, N. M. Ceglio, and D. G. Stearns, Applications of microfabrication technology to x-ray laser cavities, Journal of Vacuum Science \& Technology B 6, 2153 (1988).

[7] C. Gwyn, R. Stulen, D. Sweeney, and D. Attwood, Extreme ultraviolet lithography, Journal of Vacuum Science \& Technology B 16, 3142 (1998).

[8] D. Attwood, Soft $x$-rays and extreme ultraviolet radiation: principles and applications (Cambridge University Press, 2007).

[9] S. Bajt, D. G. Stearns, and P. A. Kearney, Investigation of the amorphousto-crystalline transition in Mo/Si multilayers, Journal of Applied Physics 90, 1017 (2001). 
[10] S. Yulin, T. Feigl, T. Kuhlmann, N. Kaiser, A. Fedorenko, V. Kondratenko, O. Poltseva, V. Sevryukova, A. Y. Zolotaryov, and E. Zubarev, Interlayer transition zones in Mo/Si superlattices, Journal of Applied Physics 92, 1216 (2002).

[11] I. Nedelcu, R. Van De Kruijs, A. Yakshin, and F. Bijkerk, Temperaturedependent nanocrystal formation in Mo/Si multilayers, Physical Review B 76, 245404 (2007).

[12] I. Nedelcu, R. Van De Kruijs, A. Yakshin, and F. Bijkerk, Thermally enhanced interdiffusion in Mo/Si multilayers, Journal of Applied Physics 103, 083549 (2008).

[13] S. Bajt, J. B. Alameda, T. W. Barbee Jr, W. M. Clift, J. A. Folta, B. Kaufmann, and E. A. Spiller, Improved reflectance and stability of Mo-Si multilayers, Optical Engineering 41, 1797 (2002).

[14] A. Yakshin, R. Van De Kruijs, I. Nedelcu, E. Zoethout, E. Louis, F. Bijkerk, H. Enkisch, and S. Müllender, Enhanced reflectance of interface engineered Mo/Si multilayers produced by thermal particle deposition, Proceedings of SPIE 65170I (2007).

[15] D. L. Windt, IMD-Software for modeling the optical properties of multilayer films, Computers in Physics 12, 360 (1998).

[16] J. Son and Y.-H. Shin, Highly c-Oriented $\mathrm{PbZr}_{0.48} \mathrm{Ti}_{0.52} \mathrm{O}_{3}$ Thin Films on Glass Substrates, Electrochemical and Solid-State Letters 12, G20 (2009).

[17] D. H. Kim, Y. K. Kim, S. Hong, Y. Kim, and S. Baik, Nanoscale bit formation in highly (111)-oriented ferroelectric thin films deposited on glass substrates for high-density storage media, Nanotechnology 22, 245705 (2011).

[18] T. Feigl, H. Lauth, S. Yulin, and N. Kaiser, Heat resistance of EUV multilayer mirrors for long-time applications, Microelectronic Engineering 57, 3 (2001).

[19] T. Böttger, D. C. Meyer, P. Paufler, S. Braun, M. Moss, H. Mai, and E. Beyer, Thermal stability of Mo/Si multilayers with boron carbide interlayers, Thin Solid Films 444, 165 (2003).

[20] I. Nedelcu, R. W. van de Kruijs, A. E. Yakshin, and F. Bijkerk, Microstructure of $\mathrm{Mo} / \mathrm{Si}$ multilayers with $\mathrm{B}_{4} \mathrm{C}$ diffusion barrier layers, Applied Optics 48, 155 (2009).

[21] S. Bruijn, R. van de Kruijs, A. Yakshin, E. Zoethout, and F. Bijkerk, Thermally induced decomposition of $B_{4} C$ barrier layers in Mo/Si multilayer structures, Surface and Coatings Technology 205, 2469 (2010).

[22] International Centre for Diffraction Data. 
[23] R. Eason, Pulsed laser deposition of thin films: applications-led growth of functional materials (John Wiley \& Sons, 2007).

[24] J.-P. Maria, H. McKinstry, and S. Trolier-McKinstry, Origin of preferential orthorhombic twinning in $\mathrm{SrRuO}_{3}$ epitaxial thin films, Applied Physics Letters 76, 3382 (2000). 


\section{Valorization and outlook}

In this thesis we describe the development of novel concepts and optical components to enhance the imaging performance at extreme ultraviolet (EUV) wavelengths. This corresponds to a potential to achieve the highest resolution in imaging known to date, i.e. a spatial resolution below $10 \mathrm{~nm}$, in applications such as photolithography and microscopy [1-3]. The investigated topics can be subdivided into two categories. A first category includes a mirror design that can be used to enhance the source quality of the EUV lithography tools. Our novel design is comprised of a diffractive Fresnel zone plate patterned in the surface of a mirror to achieve spectral purification, i.e. removing undesired wavelength bands of the EUV light sources. Simultaneously, the removed light is recycled to produce more EUV light and thus increase the overall source efficiency. The second category is a new class of multilayer mirrors with adaptive features that can be used to correct wavefront distortions, reflectance degradations or wavelength deviations. These adaptive optics are based on integrated crystalline piezoelectric thin films that can be adjusted with an externally applied voltage. Typically, the multilayer mirrors and substrates that are used in EUV applications have an amorphous structure which makes it challenging to grow high quality crystalline piezoelectric films. We developed methods to grow crystalline piezoelectric films with the highest, i.e. epitaxial, quality on amorphous structures and enhance their piezoelectric response to the highest piezoelectric response on glass substrates known to date.

The topics investigated in this thesis address physics questions which arose upon demands of the EUV lithography industry. In this sense, this research is driven by applications ultimately applied in industrial environments. In practice this research was conducted in close collaboration with the industrial partner Carl-Zeiss SMT under the project name "Smart Multilayer Interactive Optics for Lithography at Extreme UV Wavelengths (SMILE)". Some of the topics in the project were patented in view of their commercial potential. The adaptive optics concept developed in this thesis is considered a step towards enhancing the optical resolution at EUV wavelengths. Due to its high applicability and importance, the research on adaptive multilayer optics is being continued in a follow-up project that is already funded by the "Stichting Technologie en Wetenschap (STW)" of Netherlands in 2013 in collaboration with Carl-Zeiss SMT.

The thesis findings also have a strong potential to be utilized, i.e., "valorized" in a variety of other industrial and scientific applications as will be explained in addition to EUV lithography in the next paragraphs. 


\subsection{Enhancement of EUV source quality}

The light sources currently developed for EUV lithography tools are based on a laser- and discharge produced plasmas (LPP and DPP, respectively). In an LPP source a high power IR drive laser is used to generate an EUV emitting plasma. Though high EUV powers can be produced with the LPP approach, there are two challenges due to the partial reflection of the IR drive laser light from the plasma. The reflected IR light is lost for the plasma heating process while additionally causing undesired heating of the exposed photoresist materials, having followed the optical path through the EUV optics chain. In chapter 2 we presented a novel mirror design for preventing the IR light from reaching the photoresist. The design is based on a binary Fresnel zone plate that is patterned into the surface of a multilayer mirror. With this approach the undesired IR light can be removed from the exit of the light source and focused back onto the plasma. This results in a spectral purification of the EUV light by a factor of four orders of magnitude. The fraction of the IR light that is refocused onto the plasma is $35 \%$ of back reflected IR light impinging on the mirror surface. This fraction can be further increased by using multilevel Fresnel zone patterns [4]. Fabrication would follow the patterning process demonstrated before [5].

The design can also be used to focus two light beams, at different wavelengths arriving from different directions, at the same point. Such an application is timeresolved IR spectroscopy in which a UV pump pulse is used to create a transient response in the sample and the change in the absorption spectra of the sample is measured using a delayed IR probe pulse [6].

\subsection{Adaptive multilayer optics}

Though multilayer mirrors used at the EUV wavelengths reflect a major fraction, $70 \%$ at $13.5 \mathrm{~nm}$, of the incident light, the remaining $30 \%$ is absorbed. This absorbed fraction and the high average powers required in lithography applications lead to heat loads on the optics. The associated temperature changes can lead to distortion of the reflected wavefront, degradation of the mirror reflectance and deviation of the peak reflectance from the desired wavelength. We developed adaptive multilayer optics to correct for these undesired effects based on integrated crystalline piezoelectric films as shown in Fig. 7.1. In the wavefront correction scheme that is shown in Fig. 7.1(a) the piezoelectric film is integrated beneath the multilayer mirror to displace the complete multilayer mirror stack. In the reflectance tuning scheme that is shown in Fig. 7.1(b) the piezoelectric layer is integrated between two multilayer mirror stacks. The reflectance of the mirror can be adjusted by changing the thickness of the piezoelectric layer. In the wavelength tuning case shown in Fig. 7.1(c) the piezoelectric films are integrated in each bilayer of the multilayer mirror. The piezoelectric layer thicknesses can be adjusted to shift the reflectance curve to a different wavelength.

The new, adaptive multilayer optics concept is designed to be used in EUV lithography but not limited to it. The concept can be used to enhance the EUV or soft x-ray beam quality in terms of optimizing the wavefront phase, homogeneity 

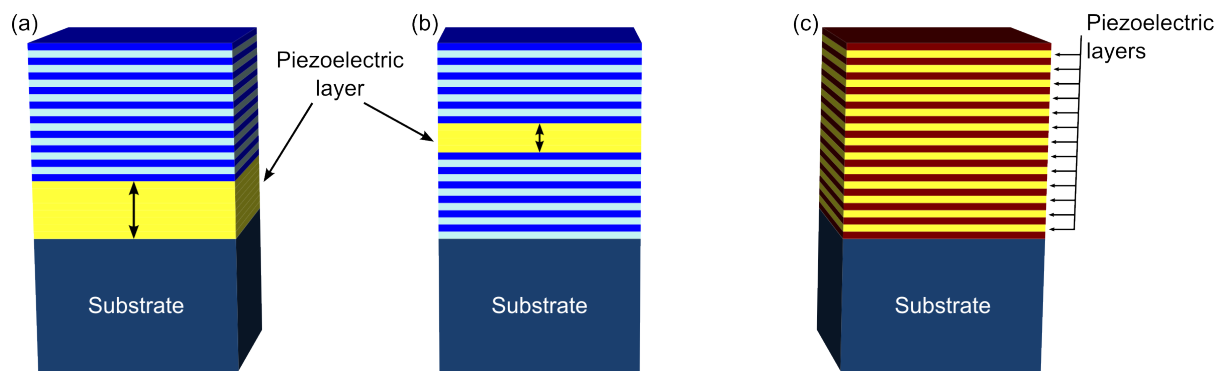

Figure 7.1: (a) A piezoelectric layer integrated beneath the multilayer mirror to move the multilayer stack vertically for shaping or correcting wavefronts. (b) A piezoelectric layer integrated between two multilayer mirror stacks to adjust the degree of interference between the beams reflected from the upper and lower multilayers, for tuning the reflectance. (c) Piezoelectric layers integrated at each period of the multilayer mirror to adjust the multilayer mirror period for tuning the wavelength or spectral response of the mirror.

of the intensity across the wavefront and matching of the spectrum of the mirror and source in areas where similar multilayer mirrors are used. Examples are free-electron laser (FEL) research, soft x-ray microscopy and astronomy [3, 7-9].

In FELs, usually grazing incidence mirrors are used that can meet the optical beam quality requirements in terms of surface figure and roughness, but the acceptance angle in a focusing configuration is very small. Multilayer mirrors equipped with the presented adaptive schemes can enable normal incidence configurations, thus large acceptance angles. This large acceptance angle combined with the close to diffraction limited wavefront qualities enable focusing high optical power into extremely small sizes that can be used to achieve unprecedented peak power densities for studying non-linear processes in extremely small length and time scales.

In the next four subsections we present the achieved results and valorization aspects of adaptive multilayer optics for (i) wavefront correction, (ii) reflectance tuning, (iii) wavelength tuning, and the required (iv) piezoelectric film growth on glass substrates.

\subsubsection{Wavefront correction}

We have demonstrated the feasibility of the wavefront correction at EUV wavelengths using the developed PZT films. Using wavefront calculations we determined the pixel size required for high-quality wavefront correction. The developed PZT films showed a high piezoelectric coefficient of $250 \mathrm{pm} / \mathrm{V}$ for the pixel sizes relevant for EUV lithography applications as demonstrated by Saathof [10]. This high piezoelectric response enables achieving the required surface displacement of $3.4 \mathrm{~nm}[10]$ with moderate voltages $(13.5 \mathrm{~V})$ that is much lower than the measured voltage limit $(100 \mathrm{~V})$ of the PZT films. This wavefront correction ap- 
proach with thin PZT films can definitely be used in EUV lithography. Compared to thermal actuation methods reported in the literature [10-12], our piezoelectric approach has the advantage of being three orders of magnitude faster. Our wavefront correction approach does not alter the spectral response of the multilayer mirror therefore it can be used at different wavelengths in applications such as soft x-ray microscopes, FELs or space telescopes [3, 7-9]. Considering the piezoelectric coefficient and the voltage limit, a maximum surface displacement of $25 \mathrm{~nm}$ can be achieved. This makes it possible to use the developed wavefront correction approach at even longer wavelengths, up to $100 \mathrm{~nm}$ considering the required stroke as quarter of the wavelength.

\subsubsection{Reflectance tuning}

We presented the design and demonstrated the feasibility of critical parameters of an adaptive multilayer mirror of which the reflectance can be tuned. The reflectance tuning is achieved by integrating a piezoelectric film between two multilayer mirror stacks. The feasibility of the surface smoothness and piezoelectric response with such a thin piezoelectric film was demonstrated experimentally. The root mean squared (RMS) surface roughness of the piezoelectric films can be decreased down to $0.3 \mathrm{~nm}$ on a substrate with $0.2 \mathrm{~nm}$ surface roughness which is an acceptable level for the EUV lithography applications. The piezoelectric film showed a piezoelectric response of $60 \mathrm{pm} / \mathrm{V}$. A dimension change of $0.5 \mathrm{~nm}$ was measured for a piezoelectric film with $30 \mathrm{~nm}$ thickness. This corresponds to a reflectance tuning range of $\sim 1 \%$. When the presented reflectance tuning mirror is spatially structured into independent regions, this tuning range can allow a spatially structured illumination and homogenization of an spatially uneven illumination.

\subsubsection{Wavelength tuning}

The last investigated adaptive scheme consists of a multilayer stack that can change its bilayer period by adjustment of a voltage applied. The change in the bilayer period translates, by the Bragg condition, into a shift in the wavelength at which the peak reflectance occurs. Individual layer thickness in such an adaptive multilayer stack is approximately a quarter of the wavelength, i.e. $3.4 \mathrm{~nm}$ for $13.5 \mathrm{~nm}$ wavelength. Achieving piezoelectric response at such small thicknesses and having acceptable levels of reflectance is still a challenge. We investigated perovskite oxides that are known to have piezoelectric properties even at small thicknesses and calculated reflectance of adaptive mirrors comprised of these perovskite materials. The reflectance values of these adaptive multilayers reach up to $49 \%$ and $54 \%$ at wavelengths $13.5 \mathrm{~nm}$ and $6.7 \mathrm{~nm}$, respectively. Compared to the maximum reflectance values of $70 \%$ and $57 \%$ at $13.5 \mathrm{~nm}$ and $6.7 \mathrm{~nm}$ wavelengths respectively these adaptive mirrors have a somewhat lower reflectance [9, 13]. 


\subsubsection{Controlled epitaxial growth of piezoelectric films on glass substrates}

Growing piezoelectric films directly on amorphous structures such as glass substrates results in polycrystalline films with reduced piezoelectric response. In order to achieve a high piezoelectric response, epitaxial growth with control on the growth orientation is needed. We achieved locally epitaxial columnar growth of piezoelectric films on glass substrates using crystalline nanosheets as buffer layers to promote the crystalline growth and control the growth orientation. The growth was performed using pulsed laser deposition, that is a proven technique for growing high quality piezoelectric films. The piezoelectric materials was $\mathrm{PbZr}_{0.52} \mathrm{Ti}_{0.48} \mathrm{O}_{3}$ (PZT) composition which is known to have the highest piezoelectric response. The developed PZT films shows a stable and high piezoelectric response of $98 \mathrm{pm} / \mathrm{V}$. This growth method can also be used in applications where crystalline growth of piezoelectric films on amorphous structures or inexpensive glass substrates is needed. Here data storage as one of such application is explained.

In this thesis we investigated the PZT thin films for their high piezoelectric response. On the other hand PZT is also a ferroelectric material with a large spontaneous polarization that can be reversed by an electric field. This spontaneous polarization is used to store information in ferroelectric random access memories $[14,15]$. Ferroelectric memory has the advantage of high density data storage compared to their traditional magnetic counterparts. On the other hand, high quality growth is usually achieved on expensive $\mathrm{SrTiO}_{3}, \mathrm{MgO}$ or $\mathrm{LaAlO}_{3}$ substrates [16-19]. Therefore our method to grow ferroelectric films on inexpensive glass substrates contributes to the ferroelectric memory applications.

The presented piezoelectric films had a columnar structure and a high piezoelectric response compared to previously reported piezoelectric films on glass. However, this high piezoelectric response is still lower than the bulk piezoelectric response, due to clamping of the PZT film by the underlying substrate. In Chapter 4 we showed that the mechanical coupling between columns has a strong impact on the clamping of the PZT film. As such the clamping can be minimized by reducing the lateral density of the columns during pulsed laser deposition of the films. In our method the columnar density was minimized by increasing the repetition rate of the deposition. We measured a high piezoelectric response, up to $280 \mathrm{pm} / \mathrm{V}$, with the PZT films deposited at high repetition rate, $20 \mathrm{~Hz}$. The method contributes to understanding of the clamping effect on the piezoelectric response and it can be utilized to enhance the piezoelectric response of the PZT films used in actuator and sensor applications [20-22]. One of such applications is ultrasound imaging where high longitudinal and reduced transverse piezoelectric response due to the observed columnar growth can be used to generate high frequency acoustic signals. Having a high piezoelectric coefficient enables operation at relatively low voltages. 


\section{Bibliography}

[1] C. Wagner and N. Harned, EUV lithography: Lithography gets extreme, Nature Photonics 4, 24 (2010).

[2] A. Sakdinawat and D. Attwood, Nanoscale X-ray imaging, Nature Photonics 4, 840 (2010).

[3] W. Chao, B. D. Harteneck, J. A. Liddle, E. H. Anderson, and D. Attwood, Soft $x$-ray microscopy at a spatial resolution better than $15 \mathrm{~nm}$, Nature $\mathbf{4 3 5}$, 1210 (2005).

[4] J. Jahns and S. J. Walker, Two-dimensional array of diffractive microlenses fabricated by thin film deposition, Applied Optics 29, 931 (1990).

[5] Y. Platonov, M. Kriese, R. Crucet, Y. Li, V. Martynov, L. Jiang, J. Rodriguez, U. Mueller, J. Daniel, S. Khatri, A. Magruder, S. Grantham, C. Tarrio, and T. B. Lucatorto, in Collector development with ir suppression and EUVL optics refurbishment at RIT (2013).

[6] H. W. Siesler, Y. Ozaki, S. Kawata, and H. Michael Heise, Near-infrared spectroscopy: Principles, instruments, applications (Wiley, 2002).

[7] E. Ziegler, A. Freund, J. Susini, G. Marot, L. Zhang, F. de Bergevin, and C. Riekel, X-ray mirrors for the European synchrotron radiation facility, Optical Engineering 29, 928 (1990).

[8] E. Segato, V. DaDeppo, S. Debei, G. Naletto, G. Cremonese, and E. Flamini, Method for studying the effects of thermal deformations on optical systems for space application, Applied Optics 50, 2836 (2011).

[9] E. Louis, A. E. Yakshin, T. Tsarfati, and F. Bijkerk, Nanometer interface and materials control for multilayer EUV-optical applications, Progress in Surface Science 86, 255 (2011).

[10] R. Saathof, Adaptive optics to counteract thermal aberrations, Ph.D. thesis, Technische Universiteit Delft, The Netherlands, 2013.

[11] S. Ravensbergen, Adaptive optics for extreme ultraviolet lithography: Actuator design and validation for deformable mirror concepts, Ph.D. thesis, Technische Universiteit Eindhoven, The Netherlands, 2012.

[12] S. Ravensbergen, P. Rosielle, and M. Steinbuch, Deformable mirrors with thermo-mechanical actuators for extreme ultraviolet lithography: Design, realization and validation, Precision Engineering 37, 353 (2013).

[13] I. A. Makhotkin, E. Zoethout, R. W. E. van de Kruijs, L. E. Yakunin, S. N., A. M. Yakunin, S. Müllender, and F. Bijkerk, Short period La/B and LaN/B multilayer mirrors for $6.8 \mathrm{~nm}$ wavelength, Optics Express 20, 11778 (2012). 
[14] J. Son and Y.-H. Shin, Highly c-Oriented $\mathrm{PbZr}_{0.48} \mathrm{Ti}_{0.52} \mathrm{O}_{3}$ Thin Films on Glass Substrates, Electrochemical and Solid-State Letters 12, G20 (2009).

[15] D. H. Kim, Y. K. Kim, S. Hong, Y. Kim, and S. Baik, Nanoscale bit formation in highly (111)-oriented ferroelectric thin films deposited on glass substrates for high-density storage media, Nanotechnology 22, 245705 (2011).

[16] L. X. Cao, Y. Xu, B. R. Zhao, L. P. Guo, L. Li, B. Xu, Y. Z. Zhang, H. Chen, A. J. Zhu, Z. H. Mai, J. H. Zhao, Y. F. Fu, and L. X. J, A structural investigation of high-quality epitaxial $\mathrm{Pb}(\mathrm{Zr}, \mathrm{Ti}) \mathrm{O}_{3}$ thin films, Journal of Physics D: Applied Physics 30, 1455 (1997).

[17] I. Vrejoiu, G. Le Rhun, L. Pintilie, D. Hesse, M. Alexe, and U. Gösele, Intrinsic ferroelectric properties of strained tetragonal $\mathrm{PbZr}_{0.2} \mathrm{Ti}_{0.8} \mathrm{O}_{3}$ obtained on layerbylayer grown, defectfree singlecrystalline films, Advanced Materials 18, 1657 (2006).

[18] G. Barucca, A. De Benedittis, A. Di Cristoforo, G. Majni, P. Mengucci, F. Leccabue, and B. E. Watts, Crystallisation of perovskite PZT films on $\mathrm{MgO}$ substrates, Thin Solid Films 319, 207 (1998).

[19] J. H. Kim and F. F. Lange, Epitaxial growth of $\mathrm{PbZr}_{0.5} \mathrm{Ti}_{0.5} \mathrm{O}_{3}$ thin films on (001) $\mathrm{LaAlO}_{3}$ by the chemical solution deposition method, Journal of Materials Research 14, 4004 (1999).

[20] D. Balma, A. Mazzalai, N. Chidambaram, C. S. Sandu, A. Neels, A. Dommann, P. Hess, D. Binz, and P. Muralt, High piezoelectric longitudinal coefficients in solgel PZT thin film multilayers, Journal of the American Ceramic Society 97, 2069 (2014).

[21] C. Emery and S. Smith, Ultrasonic imaging using a 5-MHz multilayer/singlelayer hybrid array for increased signal-to-noise ratio, IEEE Transactions on Ultrasonics, Ferroelectrics, and Frequency Control 46, 1101 (1999).

[22] M. Zipparo, K. Bing, and K. Nightingale, Imaging arrays with improved transmit power capability, IEEE Transactions on Ultrasonics, Ferroelectrics, and Frequency Control 57, 2076 (2010). 



\section{A Wavelength tuning}

Multilayer mirrors (MLMs) are widely used in extreme ultraviolet (EUV) and soft x-ray wavelengths for applications such as photolithography, microscopy, astronomy and free electron laser research [1]. The MLMs are fabricated by depositing many pairs of high and low refractive index materials to combine partial reflections occurring due to the refractive index difference. For the design of MLMs, usually a material with very low absorption and another material that has a high refractive index contrast with the spacer layer is selected. These low absorptive and high refractive index materials are usually referred as spacer and absorber layers, respectively. The total thickness of an absorber and a spacer layer pair, i.e. bilayer period, and the ratio of the layer thicknesses determines the wavelength of maximum reflectance according to the Bragg condition [1]. A major fraction of the incoming light can be reflected using MLMs designed according to the Bragg condition, but also part of the incoming light is absorbed by the layers. This absorbed EUV light leads to a significant heat load on the MLM. The temperature changes induced by the heat load can cause deformation of the substrate and multilayer mirror. Due to the extremely small wavelength, down to sub-10 nm, even minor changes in the MLM thickness can lead to significant deviations from the desired wavelength response of the MLM.

In this appendix we present an MLM concept that can be adapted to wavelength deviations by changing its layer thickness. In this adaptive MLM design, at least one of the layer is chosen as a piezoelectric material that can be expanded by applying an external voltage. To demonstrate the principle and quantify the shift of the reflectance curve, a hypothetical example with a standard MLM is explained in Fig. A.1. The solid reflectance curve shown in Fig. A.1 is the reflectance curve of a Mo/Si MLM that is designed for maximum reflectance around $13.5 \mathrm{~nm}$ at normal incidence. In the MLM, the bilayer period is $6.9 \mathrm{~nm}$, the ratio of the absorber thickness to the bilayer period is 0.4 and the number of bilayers is 50 . With these settings, the maximum reflectance is $\sim 75 \%$ at $13.5 \mathrm{~nm}$. When the absorber Mo layer is expanded hypothetically by $1 \%$, which can be achieved by piezoelectric materials [2], the reflectance curve shifts to a longer wavelength, $13.6 \mathrm{~nm}$, as shown with dashed line in Fig. A.1. The wavelength shift is significant considering the EUV wavelength and comparable to the expansion of the absorber layer, approximately $1 \%$. There is no significant change in the maximum reflectance. After this demonstration of the principle, adaptive MLMs in which the layers are selected from piezoelectric materials will be explained.

In a realistic MLM with piezoelectric materials, the reflectance becomes an important parameter to be investigated. Here, we considered the materials taken from a crystallography database [3-5] as the MLM components and calculated their refractive indices based on their density, molecular composition and atomic 


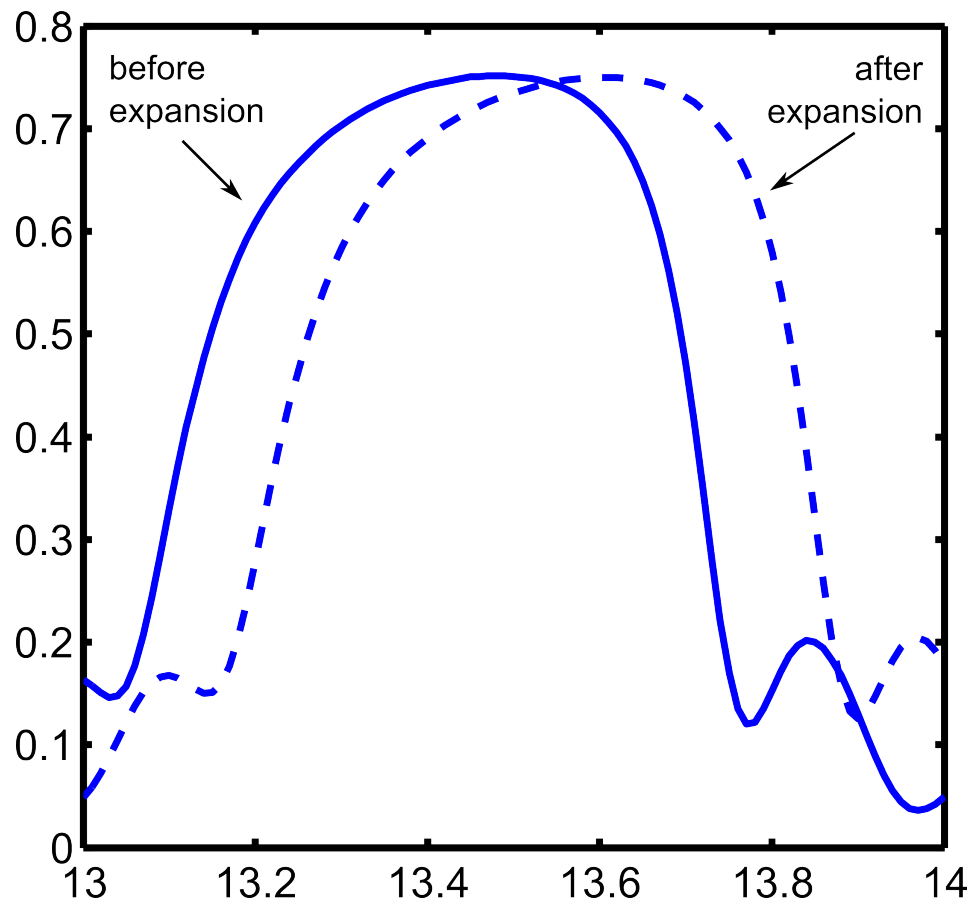

Figure A.1: Shift of the reflectance curve after expansion of the absorber layer by $1 \%$.

scattering factors [1]. Using the calculated refractive indices, the reflectance of the adaptive MLMs were computed [6] at two major wavelengths $13.5 \mathrm{~nm}$ and $6.7 \mathrm{~nm}$ that are important for lithography applications. The thickness of a single layer is approximately quarter of the wavelength, which corresponds to $1.7 \mathrm{~nm}$ for $6.7 \mathrm{~nm}$ wavelength. With such small thickness the layers should likely to be grown completely from crystalline materials to have a high quality piezoelectric layer that can have a piezoelectric response. Here we investigated perovskite oxides that are known to have piezoelectric response at such thicknesses and studied extensively for their remarkably high piezoelectric response [7]. The perovskite oxides has a chemical formula of $\mathrm{ABO}_{3}$, where $\mathrm{A}$ and $\mathrm{B}$ are cations. The number of perovskite oxides that were found in the crystallography database was large, more than 600 . We utilized these perovskite materials as the absorber layer, and kept $\mathrm{Si}$ and $\mathrm{B}$ as the spacer layer for $13.5 \mathrm{~nm}$ and $6.7 \mathrm{~nm}$, respectively. The MLMs that have reflectance values higher than $40 \%$ are presented in Table A.1. The reflectance reach up to $49 \%$ and $53.5 \%$ at wavelengths $13.5 \mathrm{~nm}$ and $6.7 \mathrm{~nm}$, respectively. 


\section{Bibliography}

[1] D. Attwood, Soft $x$-rays and extreme ultraviolet radiation: principles and applications (Cambridge University Press, 2007).

[2] M. Bayraktar, A. Chopra, G. Rijnders, K. Boller, and F. Bijkerk, Wavefront correction in the extreme ultraviolet wavelength range using piezoelectric thin films, Optics Express 22, 30623 (2014).

[3] S. Graulis, A. Dakevi, A. Merkys, D. Chateigner, L. Lutterotti, M. Quirs, N. R. Serebryanaya, P. Moeck, R. T. Downs, and A. Le Bail, Crystallography Open Database (COD): an open-access collection of crystal structures and platform for world-wide collaboration, Nucleic Acids Research 40, D420 (2012).

[4] S. Grazulis, D. Chateigner, R. T. Downs, A. T. Yokochi, M. Quiros, L. Lutterotti, E. Manakova, J. Butkus, P. Moeck, and A. Le Bail, Crystallography Open Database - an open-access collection of crystal structures, Journal of Applied Crystallography 42, 726 (2009).

[5] R. T. Downs and M. Hall-Wallace, The american mineralogist crystal structure database, American Mineralogist 88, 247 (2003).

[6] I. V. Kozhevnikov, R. van der Meer, H. Bastiaens, K.-J. Boller, and F. Bijkerk, Analytic theory of soft $x$-ray diffraction by lamellar multilayer gratings, Optics Express 19, 9172 (2011).

[7] D. D. Fong, G. B. Stephenson, S. K. Streiffer, J. A. Eastman, O. Auciello, P. H. Fuoss, and C. Thompson, Ferroelectricity in ultrathin perovskite films, Science 304, 1650 (2004). 
Table A.1: Reflectance of spectrally adaptive multilayer mirrors at $13.5 \mathrm{~nm}$ and 6.7 nm wavelengths.

\begin{tabular}{|c|c|c|c|}
\hline $\begin{array}{l}\text { Wavelength } \\
(\mathrm{nm})\end{array}$ & Absorber & $\begin{array}{l}\text { Density } \\
\left(\mathrm{g} / \mathrm{cm}^{3}\right)\end{array}$ & $\begin{array}{l}\text { Reflectance } \\
\%\end{array}$ \\
\hline 13.5 & $\mathrm{SrCa}_{0.33} \mathrm{Nb}_{0.67} \mathrm{O}_{3}$ & 5.09 & 49.0 \\
\hline (Spacer layer: Si, & $\mathrm{CaIrO}_{3}$ & 8.62 & 46.0 \\
\hline \multirow[t]{11}{*}{ Density: $2.33 \mathrm{~g} / \mathrm{cm}^{3}$ ) } & $\mathrm{YMnO}_{3}$ & 5.15 & 45.8 \\
\hline & $K_{0.3} \mathrm{Na}_{0.7} \mathrm{NbO}_{3}$ & 4.50 & 43.7 \\
\hline & $\mathrm{Mn}_{2} \mathrm{O}_{3}$ & 5.03 & 43.5 \\
\hline & $K_{0.05} \mathrm{Na}_{0.95} \mathrm{NbO}_{3}$ & 4.51 & 42.1 \\
\hline & $L i_{0.02} N a_{0.98} N b O_{3}$ & 4.61 & 42.1 \\
\hline & $\mathrm{CaCO}_{3}$ & 2.57 & 41.4 \\
\hline & $\mathrm{PbZn}_{0.31} \mathrm{Nb}_{0.61} T i_{0.08} \mathrm{O}_{3}$ & 8.39 & 40.9 \\
\hline & $\mathrm{Tl}_{2} \mathrm{O}_{3}$ & 10.3 & 40.5 \\
\hline & $\mathrm{PbH}_{0.4} \mathrm{Ti}_{0.6} \mathrm{O}_{3}$ & 9.67 & 40.4 \\
\hline & $\mathrm{FeMnO}_{3}$ & 5.16 & 40.2 \\
\hline & $\mathrm{PbVO}_{3}$ & 7.54 & 40.0 \\
\hline 6.7 & $L a_{2} O_{3}$ & 6.61 & 53.5 \\
\hline (Spacer layer: B, & $\mathrm{Ce}_{2} \mathrm{O}_{3}$ & 6.90 & 45.4 \\
\hline Density: $2.37 \mathrm{~g} / \mathrm{cm}^{3}$ ) & $\mathrm{Pr}_{2} \mathrm{O}_{3}$ & 7.11 & 41.5 \\
\hline
\end{tabular}




\section{B Controlled growth of $\mathrm{PbZr}_{0.52} \mathrm{Ti}_{0.48} \mathrm{O}_{3}$ using nanosheet coated Si (001)}

Preferentially (001) and (110)-oriented $\mathrm{PbZr}_{0.52} \mathrm{Ti}_{0.48} \mathrm{O}_{3}$ (PZT) films with a $\mathrm{LaNiO}_{3}$ (LNO) bottom electrode were deposited on buffered Si (001) substrates using pulsed laser deposition. This high degree of control on growth orientation of these LNO and PZT thin films was achieved by using $\mathrm{Ca}_{2} \mathrm{Nb}_{3} \mathrm{O}_{10}$ (CNO) and $\mathrm{Ti}_{0.87} \mathrm{O}_{2}$ (TO) nanosheets as buffer layers deposited on the $\mathrm{Si}$ by the LangmuirBlodgett technique. The measured remnant polarization and piezoelectric response of the (001)-oriented PZT films on CNO-nanosheets are $16 \mathrm{\mu C} / \mathrm{cm}^{2}$ and $120 \mathrm{pm} / \mathrm{V}$, respectively. These values are comparable to the values reported for the epitaxial $\mathrm{PZT}$ films grown on $\mathrm{CeO}_{2} /$ yttriastabilized zirconia (YSZ) buffered Si substrates while the maximum deposition temperature is lowered from $800^{\circ} \mathrm{C}$ to $600^{\circ} \mathrm{C}$ which are the typical deposition temperatures of YSZ and PZT respectively. This lower maximum deposition temperature shows that the integration of PZT films by the nanosheet buffer layers is not limited to $\mathrm{Si}$, but can be extended to substrates with low processing temperatures like glass for various device applications in the future. 


\section{B.1 Introduction}

In recent years lead zirconate titanate, $\mathrm{PbZr}_{x} \mathrm{Ti}_{1-x} \mathrm{O}_{3}(\mathrm{PZT})$, films have drawn much attention because of their potential use in microelectromechnical systems (MEMS) [1] and nonvolatile ferroelectric random access memories [2]. The properties of PZT films strongly depend on the composition (Zr/Ti ratio) therefore PZT (52/48) composition near the morphotropic phase boundary (MPB) is commonly preferred due to its high piezoelectric response [3]. Moreover in order to utilize the optimal functional properties of PZT, high crystalline quality films with control on crystal-orientation is required [4]. Therefore, high quality epitaxial PZT films are usually prepared on single crystal oxide substrates such as $\mathrm{SrTiO}_{3}, \mathrm{MgO}$ and $\mathrm{LaAlO}_{3}$ using various physical and chemical vapor deposition techniques [5-8]. However, these substrates are expensive and therefore rarely used in practical applications. In the semiconductor industry, silicon ( $\mathrm{Si}$ ) substrates are commonly used, necessitating high quality growth of PZT on Si or Si-containing substrates. Integration of PZT on Si substrates and circuitry could pave the way to more efficient fabrication of devices. However, growth of oxide films, including PZT, directly on Si causes oxidation of the Si surface forming amorphous silicon oxide at the film-substrate interface, which is detrimental for growth of high quality crystalline films. As a result considerable efforts have been invested to find buffer layers that prevent such oxidation at the Si surface, allowing control of the crystal orientation of the subsequent layers. One way of growing oxides on $\mathrm{Si}$ is depositing yttria-stabilized zirconia (YSZ) and then $\mathrm{CeO}_{2}$ as buffer layers [9-12]. There are also efforts to integrate preferentially oriented PZT on platinized Si substrates at lower temperatures, but issues like control of the growth orientation and fatigue are still to be overcome for a stable and reproducible response of PZT [13, 14]. Ferroelectric and piezoelectric properties are highly anisotropic, thus integration of PZT on Si with control of the growth orientation is highly desired [4]. Orientation-control on growth direction is usually achieved by using $\mathrm{SrRuO}_{3}(\mathrm{SRO})$ or $\mathrm{LaNiO}_{3}$ (LNO) electrodes on YSZ and/or $\mathrm{CeO}_{2}$ buffer layers. However, growth of $\mathrm{YSZ}$ and $\mathrm{CeO}_{2}$ on $\mathrm{Si}$ is usually achieved at very high temperatures $\left(750-800^{\circ} \mathrm{C}\right)[9-12]$ which would create problems for any circuitry on the same substrate. In addition, such a high temperature growth process is not very suitable for the integration of PZT on substrates like glass. Hence in order to integrate PZT on cost effective substrates such as Si and glass, development of new integration processes with lower processing temperature is required.

Recently two-dimensional materials such as oxide nanosheets have attracted attention due to their novel physical properties and potential use in a wide range of applications such as dielectrics and semiconductors [15-18]. The advantage of the nanosheets is that they can be transferred in crystalline form to the substrates such as Si and glass at room temperature. Contrary to the major attention from the dielectric and semiconductor applications, little attention has been paid on the potential use of the nanosheets as a buffer/seed layer to promote piezoelectric film growth $[19,20]$. On the other hand, these works $[19,20]$ use chemical solution deposition for growth of Ti rich compositions of PZT and the produced films suf- 
fer from mixed growth and high coercive fields. In addition, no data is reported regarding the piezoelectric properties of the PZT films, which is indeed crucial for the device applications. To our knowledge, there is no detailed investigation on control of growth direction of PZT (52/48) films using $\mathrm{Ca}_{2} \mathrm{Nb}_{3} \mathrm{O}_{10}$ (CNO) and $\mathrm{Ti}_{0.87} \mathrm{O}_{2}$ (TO) nanosheets as buffer layers on Si substrates. In this letter, we report the controlled growth of the (001) and (110)-oriented $\mathrm{PZT} / \mathrm{LaNiO}_{3}$ (LNO) films using CNO and TO nanosheets as buffer layers, respectively on Si substrates. Comparable ferroelectric and piezoelectric properties were measured for both (001) and (110)-oriented PZT films. We used pulsed laser deposition (PLD) which is known to give better control on the stoichiometry and crystal orientation. The entire growth process was achieved at temperatures lower than $600{ }^{\circ} \mathrm{C}$, which makes nanosheets attractive buffer layers for the integration of $\mathrm{PZT}$ also on glass.

\section{B.2 Experimental procedure}

$\mathrm{CNO}$ and TO-nanosheet films were transferred from a single layer CNO and TO colloidal solution using the LangmuirBlodgett (LB) technique at room temperature. The solutions of CNO and TO-nanosheets were prepared by exfoliation of layered $\mathrm{HCa}_{2} \mathrm{Nb}_{3} \mathrm{O}_{10} \cdot 1.5 \mathrm{H}_{2} \mathrm{O}$ and $\mathrm{H}_{1.7} \mathrm{Ti}_{1.73} \mathrm{O}_{4} \cdot \mathrm{H}_{2} \mathrm{O}$ respectively, by using tetrabutylammonium hydroxide $(\mathrm{TBAOH})$ [18-21]. The nanosheet films with layer number of $>3$ are not stable at temperatures higher than $650{ }^{\circ} \mathrm{C}[22]$, thus only 1 layer of $\mathrm{CNO}$ and 2 layers of TO-nanosheets were deposited on Si substrates. Prior to the deposition of the PZT/LNO stack, the nanosheet buffered Si (001) substrates were annealed at $600{ }^{\circ} \mathrm{C}$ for 60 minutes in a 0.140 mbar oxygen atmosphere. This step was required to burn out the surfactant used to grow the nanosheets and increase the adhesion between substrate and nanosheets. Figure B.1(a) and (b) show $20 \times 20 \mathrm{\mu m}^{2}$ atomic force microscopy (AFM) images of the CNO and TO-nanosheets. The AFM images show successful deposition of $\mathrm{CNO}$ and TO nanosheets on Si substrates with high area coverage.
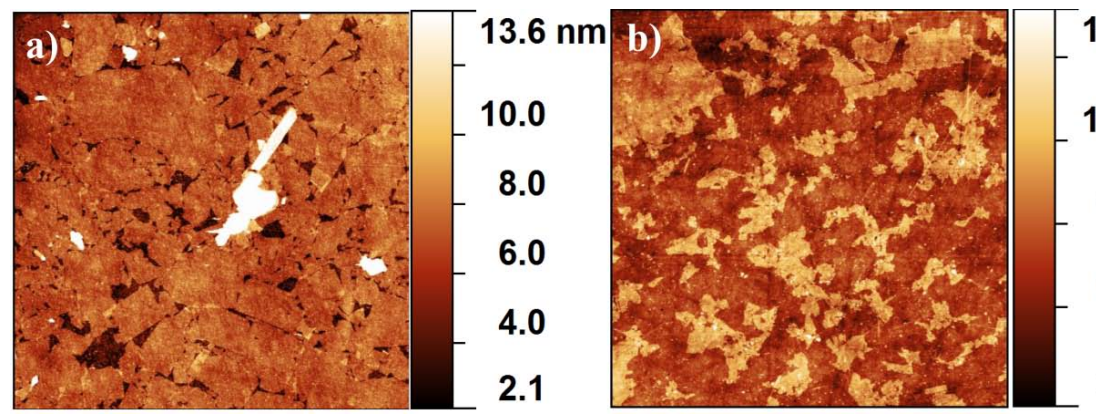

$13.2 \mathrm{~nm}$

10.0

8.0

6.0

3.6

Figure B.1: AFM images recorded in $20 \times 20 \mathrm{\mu m}^{2}$ area for (a) $\mathrm{Ca}_{2} \mathrm{Nb}_{3} \mathrm{O}_{10}$ and (b) $\mathrm{Ti}_{0.87} \mathrm{O}_{2}$-nanosheets revealing high area coverage. 
All films were deposited in-situ using PLD by ablating materials from stoichiometric targets of LNO and PZT with KrF excimer laser pulses at $248 \mathrm{~nm}$ wavelength with $20 \mathrm{~ns}$ pulse duration. A base pressure of $5 \times 10^{-7}$ mbar was maintained in the deposition chamber before raising the substrate temperature. PZT films with $750 \mathrm{~nm}$ thickness were sandwiched between $200 \mathrm{~nm}$ thick bottom and $100 \mathrm{~nm}$ thick top LNO electrodes respectively. In order to avoid any degradation of PZT phase, the top LNO electrode layer was deposited at the same growth temperature as of $\mathrm{PZT}\left(585^{\circ} \mathrm{C}\right)$. Later on, $100 \mathrm{~nm}$ thick $\mathrm{Pt}$ was sputtered on the LNO top electrode by radio frequency sputtering at room temperature to increase the homogeneity of the electric field. After the depositions, top electrode was patterned into capacitors with $200 \times 200 \mathrm{\mu m}^{2}$ area using standard photolithography process and structured by argon etching.

Crystallographic characterization was performed by means of a Philips X'Pert MRD X-ray diffractometer using $\mathrm{Cu}-\mathrm{K} \alpha$ radiation. The scanning electron microscopy images of both of the heterostructures were recorded using a Zeiss MERLIN HR-SEM. Polarization-electric field $(P-E)$ hysteresis loop and switching current-electric field $(I-E)$ characteristics of the PZT films were recorded with a ferroelectric tester (TF analyzer 2000, aixACCT). The ferroelectric hysteresis loops were measured using bipolar triangular pulses at $1 \mathrm{kHz}$. The piezoelectric response of the films was measured using a Polytec MSA-400 laser Doppler vibrometer (LDV) operating at $8 \mathrm{kHz}$.

\section{B.3 Results and discussion}

The XRD patterns of PZT and LNO films on CNO and TO-nanosheet buffered Si (001) substrates (pseudo-cubic indexing is used for all) are shown in Fig. B.2(a). The PZT films consist of a pure perovskite phase and no pyrochlore phase was observed in both heterostructures. Figure B.2(a) shows that PZT films grown on CNO and TO-nanosheets have (001)-orientation and (110)-orientation, respectively. The CNO-nanosheet had a $2 \mathrm{D}$ square lattice with a lattice parameter of $a=3.86 \AA$, which is an exact fit to that of the (001) face of the cubic LNO $(a=3.86 \AA)$. This exact fit of lattice parameters promotes (001)-growth of both LNO and PZT on CNO-nanosheets as schematically shown in Fig. B.2(b-c) and observed in the XRD patterns. TO-nanosheets have a lepidocrocite-type structure with a $2 \mathrm{D}$ rectangular lattice with lattice parameter of $a=3.76 \AA$ and $a=2.97 \AA$ [17]. The two-fold cell of a TO-nanosheet provides a close match to the (110) unit cell of LNO as schematically shown in Fig. B.2(d-e). This promotes (110)-oriented growth of both LNO and PZT. However, nanosheets are randomly oriented in-plane, thus the subsequent layers are also expected to be randomly oriented in-plane. The microstructure of the PZT films deposited on $\mathrm{CNO}$ and TO-nanosheet buffered Si substrate was further investigated by SEM as shown in Fig. B.3(a) and (b), respectively. Cross-sectional SEM images reveal thicknesses around $600 \mathrm{~nm}$ and $200 \mathrm{~nm}$ for the PZT film and LNO electrode layer, respectively. It has been observed from the SEM images that PZT films show a columnar type of growth for both heterostructures due to the large lattice 
mismatch ( $5 \%$ ) between LNO and PZT.
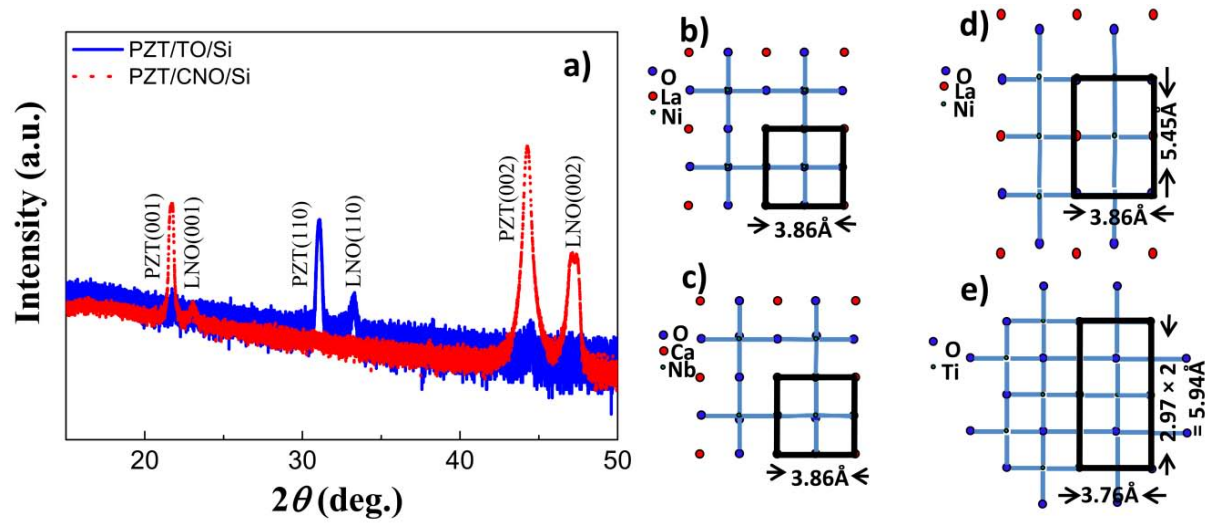

Figure B.2: (a) XRD $\theta-2 \theta$ scan of PZT films revealing (001) and (110)-orientation on $\mathrm{Ca}_{2} \mathrm{Nb}_{3} \mathrm{O}_{10} / \mathrm{Si}$ and $\mathrm{Ti}_{0.87} \mathrm{O}_{2} / \mathrm{Si}$ substrates. Since the nanosheets are only a few unit cells thick, they are not visible in the XRD measurements. A schematic demonstration of (b-c) the ideal fitting of the $\mathrm{LaNiO}_{3}$ (100) plane lattice parameters to the $\mathrm{Ca}_{2} \mathrm{Nb}_{3} \mathrm{O}_{10}$ lattice parameters, (d-e) the match of $\mathrm{LaNiO}_{3}$ (110) plane lattice parameters and $\mathrm{Ti}_{0.87} \mathrm{O}_{2}$ lattice parameters.

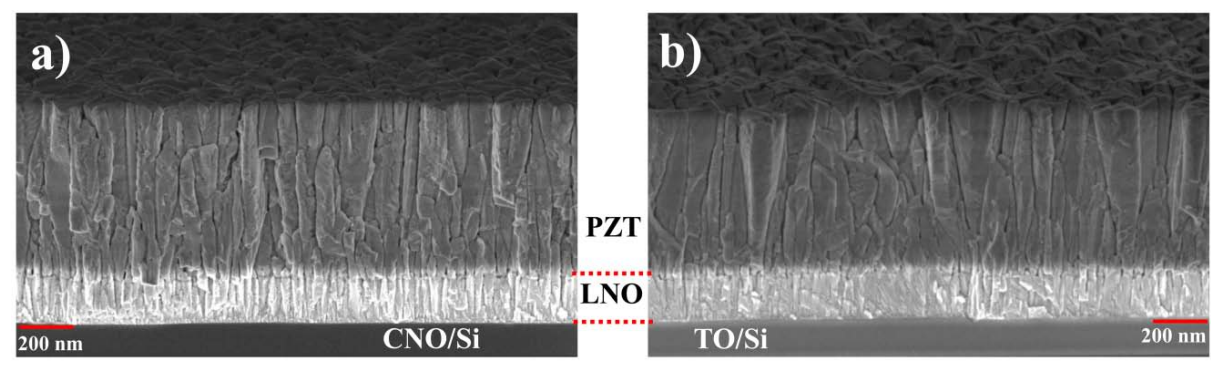

Figure B.3: SEM images of PZT films on (a) LNO/CNO/Si and (b) LNO/TO/Si.

The polarization-electric field $(P-E)$ hysteresis and switching current-electric field $(I-E)$ which were measured at $1 \mathrm{kHz}$ for two heterostructures are shown in Fig. B.4(a) and (b) respectively. Despite having different crystal-orientations for PZT films remnant polarization $\left(P_{r}\right)$ and coercive field $\left(E_{c}\right)$ for both of the heterostructures are comparable. The remnant polarization $\left(P_{r}\right)$, and coercive field $\left(E_{c}\right)$ for both structures are $16 \mu \mathrm{C} / \mathrm{cm}^{2}$ and $32 \mathrm{kV} / \mathrm{cm}$ respectively, which is comparable to the values reported for the epitaxial PZT films on buffered Si substrates [12]. Well saturated hysteresis loops and sharp switching peaks observed in ferroelectric measurements show that the quality of these PZT films on nanosheets is comparable to the quality of epitaxial PZT films reported on 
YSZ $\backslash \mathrm{CeO}_{2}$ buffered Si substrates [12]. Furthermore, the piezoelectric response of the PZT film was locally measured using LDV. The samples were fixed to large metal plates with silver paste to prevent any contribution of the bending of the substrates to the LDV measurements. The piezoelectric hysteresis loops shown in Fig. B.5 were measured at electrodes with $200 \times 200 \mathrm{\mu m}^{2}$ area in response to a small AC electric field of $6.7 \mathrm{kV} / \mathrm{cm}$. This AC electric field was superimposed on a DC electric field sweeping from $-170 \mathrm{kV} / \mathrm{cm}$ to $170 \mathrm{kV} / \mathrm{cm}$. The maximum piezoelectric response $\left(d_{33}\right)$ measured for PZT films on CNO and TO-nanosheets are $120 \mathrm{pm} / \mathrm{V}$ and $108 \mathrm{pm} / \mathrm{V}$, respectively. These values are higher as compared to the values reported for the PZT films by LDV [23], which shows the high quality of PZT films on nanosheets buffered Si substrates.
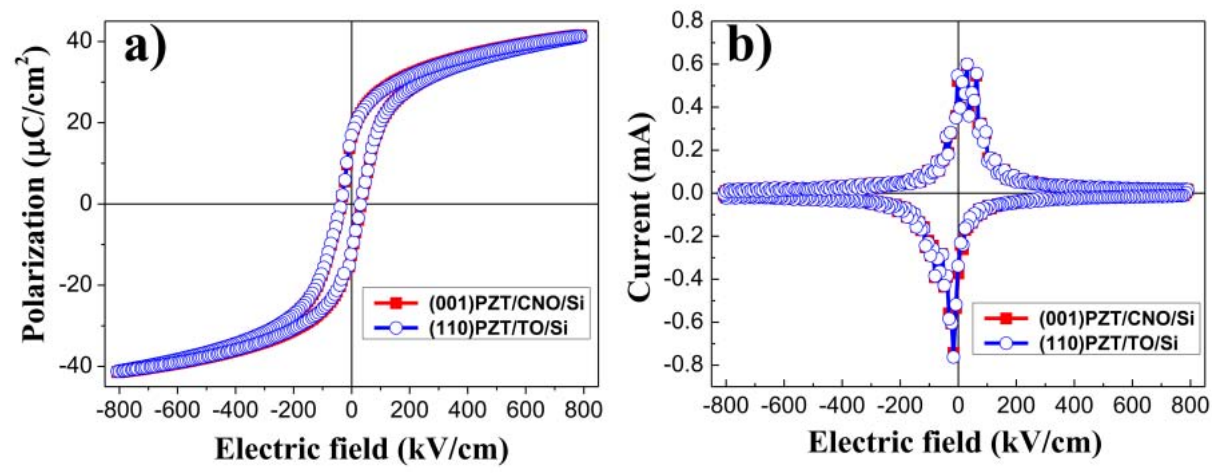

Figure B.4: (a) Macroscopic polarization-electric field hysteresis and (b) switching current-electric field curves of PZT films on $\mathrm{Ca}_{2} \mathrm{Nb}_{3} \mathrm{O}_{10}$ and $\mathrm{Ti}_{0.87} \mathrm{O}_{2}$-nanosheets revealing that PZT films could be switched in either direction.

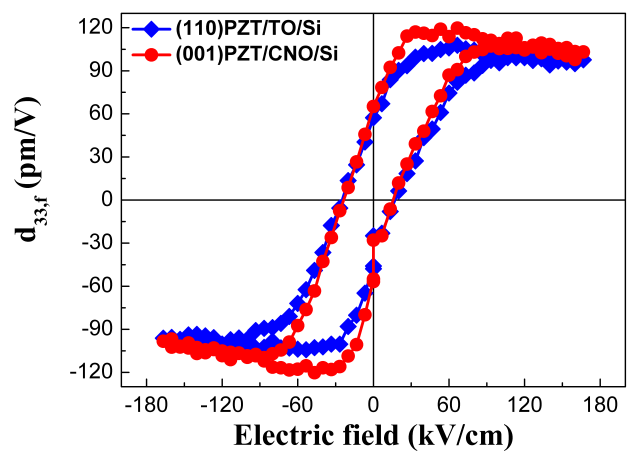

Figure B.5: The piezoelectric hysteresis $\left(d_{33}\right)$ loops measured by LDV for PZT films on $\mathrm{Ca}_{2} \mathrm{Nb}_{3} \mathrm{O}_{10}$ and $\mathrm{Ti}_{0.87} \mathrm{O}_{2}$-nanosheets. 


\section{B.4 Conclusions}

In summary, a high degree of control on the crystal growth orientation of PZT films on Si substrates is achieved by incorporating CNO and TO-nanosheets. The results reveal that the PZT films deposited on nanosheets have comparable ferroelectric properties, whereas superior piezoelectric properties were obtained as compare to the films reported in the literature. This paper shows that integration of PZT films at relatively low temperature with control on crystal-orientation to the Si-based devices is possible, which is expected to have a positive impact on many practical applications of piezoelectric films.

\section{Acknowledgements}

This research program is funded by "Stichting Technologie en Wetenschap (STW)" under the contract 10448 with the project name "Smart Multilayer Interactive Optics for Lithography at Extreme UV wavelengths (SMILE)". The authors would like to thank Prof. J. E. ten Elshof and Mr. Maarten Nijland for providing the nanosheets and Dr. Minh Nguyen for Pt coating.

\section{Bibliography}

[1] S. Trolier-McKinstry and P. Muralt, Thin film piezoelectrics for MEMS, Journal of Electroceramics 12, 7 (2004).

[2] Ferroelectric thin films, edited by M. Okuyama and Y. Ishibashi (SpringerVerlag, Berlin Heidelberg, 2005).

[3] B. Jaffe, W. R. Cook, and H. Jaffe, Piezoelectric ceramics (Academic, London, 1971).

[4] X.-h. Du, J. Zheng, U. Belegundu, and K. Uchino, Crystal orientation dependence of piezoelectric properties of lead zirconate titanate near the morphotropic phase boundary, Applied Physics Letters 72, 2421 (1998).

[5] L. X. Cao, Y. Xu, B. R. Zhao, L. P. Guo, L. Li, B. Xu, Y. Z. Zhang, H. Chen, A. J. Zhu, Z. H. Mai, J. H. Zhao, Y. F. Fu, and L. X. J, A structural investigation of high-quality epitaxial $\mathrm{Pb}(\mathrm{Zr}, \mathrm{Ti}) \mathrm{O}_{3}$ thin films, Journal of Physics D: Applied Physics 30, 1455 (1997).

[6] I. Vrejoiu, G. Le Rhun, L. Pintilie, D. Hesse, M. Alexe, and U. Gösele, Intrinsic ferroelectric properties of strained tetragonal $\mathrm{PbZ}_{0.2} \mathrm{Ti}_{0.8} \mathrm{O}_{3}$ obtained on layerbylayer grown, defectfree singlecrystalline films, Advanced Materials 18, 1657 (2006).

[7] G. Barucca, A. De Benedittis, A. Di Cristoforo, G. Majni, P. Mengucci, F. Leccabue, and B. E. Watts, Crystallisation of perovskite PZT films on $\mathrm{MgO}$ substrates, Thin Solid Films 319, 207 (1998). 
[8] J. H. Kim and F. F. Lange, Epitaxial growth of $\mathrm{PbZr}_{0.5} \mathrm{Ti}_{0.5} \mathrm{O}_{3}$ thin films on (001) $\mathrm{LaAlO}_{3}$ by the chemical solution deposition method, Journal of Materials Research 14, 4004 (1999).

[9] D. K. Fork, D. B. Fenner, G. A. N. Connell, J. M. Phillips, and T. H. Geballe, Epitaxial yttria-stabilized zirconia on hydrogen-terminated Si by pulsed laser deposition, Applied Physics Letters 57, 1137 (1990).

[10] S. J. Wang, C. K. Ong, L. P. You, and S. Y. Xu, Epitaxial growth of yittriastabilized zirconia oxide thin film on natively oxidized silicon wafer without an amorphous layer, Semiconductor Science and Technology 15, 836 (2000).

[11] A. Chopra, D. Pantel, Y. Kim, M. Alexe, and D. Hesse, Microstructure and ferroelectric properties of epitaxial cation ordered $\mathrm{PbSc}_{0.5} \mathrm{Ta}_{0.5} \mathrm{O}_{3}$ thin films grown on electroded and buffered Si(100), Journal of Applied Physics 114, 084107 (2013).

[12] M. Dekkers, M. D. Nguyen, R. Steenwelle, P. M. te Riele, D. H. A. Blank, and G. Rijnders, Ferroelectric properties of epitaxial $\mathrm{Pb}(\mathrm{Zr}, \mathrm{Ti}) \mathrm{O}_{3}$ thin films on silicon by control of crystal orientation, Applied Physics Letters 95, 012902 (2009).

[13] J. G. E. Gardeniers, A. Smith, and C. Cobianu, Characterisation of sol-gel PZT films on Pt-coated substrates, Journal of Micromechanics and Microengineering 5, 153 (1995).

[14] A. Kumar, M. Alam, A. Mangiaracina, and M. Shamsuzzoha, Synthesis of the PZT films deposited on Pt-coated (100) Si substrates for nonvolatile memory applications, Journal of Electronic Materials 26, 1331 (1997).

[15] M. Osada, K. Akatsuka, Y. Ebina, Y. Kotani, K. Ono, H. Funakubo, S. Ueda, K. Kobayashi, K. Takada, and T. Sasaki, Langmuir-Blodgett fabrication of nanosheet-based dielectric films without an interfacial dead layer, Japanese Journal of Applied Physics 47, 7556 (2008).

[16] R. Ma and T. Sasaki, Nanosheets of oxides and hydroxides: Ultimate 2D charge-bearing functional crystallites, Advanced Materials 22, 5082 (2010).

[17] T. Shibata, H. Takano, Y. Ebina, D. S. Kim, T. C. Ozawa, K. Akatsuka, T. Ohnishi, K. Takada, T. Kogure, and T. Sasaki, Versatile van der Waals epitaxy-like growth of crystal films using two-dimensional nanosheets as a seed layer: orientation tuning of $\mathrm{SrTiO}_{3}$ films along three important axes on glass substrates, Journal of Material Chemistry C 2, 441 (2014).

[18] M. Nijland, S. Kumar, R. Lubbers, D. H. A. Blank, G. Rijnders, G. Koster, and J. E. ten Elshof, Local control over nucleation of epitaxial thin films by seed layers of inorganic nanosheets, ACS Applied Materials \& Interfaces 6, 2777 (2014). 
[19] K. Kikuta, K. Noda, S. Okumura, T. Yamaguchi, and S.-i. Hirano, Orientation control of perovskite thin films on glass substrates by the application of a seed layer prepared from oxide nanosheets, Journal of Sol-Gel Science and Technology 42, 381 (2007).

[20] Y. Minemura, K. Nagasaka, T. Kiguchi, T. J. Konno, H. Funakubo, and H. Uchida, Fabrication and evaluation of one-axis oriented lead zirconate titanate films using metal-oxide nanosheet interface layer, Japanese Journal of Applied Physics 52, 09KA04 (2013).

[21] A. Kumar, S. K. C. Palanisamy, J. M. Boter, C. Hellenthal, J. E. ten Elshof, and $\mathrm{H}$. J. W. Zandvliet, Imaging of $\mathrm{Ti}_{0.87} \mathrm{O}_{2}$ nanosheets using scanning tunneling spectroscopy, Applied Surface Science 265, 201 (2013).

[22] K. Fukuda, Y. Ebina, T. Shibata, T. Aizawa, I. Nakai, and T. Sasaki, Unusual crystallization behaviors of anatase nanocrystallites from a molecularly thin titania nanosheet and its stacked forms: Increase in nucleation temperature and oriented growth, Journal of the American Chemical Society 129, 202 (2007).

[23] S. Sivaramakrishnan, P. Mardilovich, A. Mason, A. Roelofs, T. SchmitzKempen, and S. Tiedke, Electrode size dependence of piezoelectric response of lead zirconate titanate thin films measured by double beam laser interferometry, Applied Physics Letters 103, 132904 (2013). 



\section{Zernike - Fringe polynomials}

The Zernike - Fringe polynomials that are used in chapter 5 are listed below. $\varphi$ is the angle measured counterclockwise and $\rho$ is the normalized radial coordinate. Visualization of the polynomials with unity amplitude is presented in Fig. C.1.

$$
\begin{aligned}
& Z_{1}=1 \\
& Z_{2}=\rho \cos (\varphi) \\
& Z_{3}=\rho \sin (\varphi) \\
& Z_{4}=2 \rho^{2}-1 \\
& Z_{5}=\rho^{2} \cos (2 \varphi) \\
& Z_{6}=\rho^{2} \sin (2 \varphi) \\
& Z_{7}=\left(3 \rho^{2}-2\right) \rho \cos (\varphi) \\
& Z_{8}=\left(3 \rho^{2}-2\right) \rho \sin (\varphi) \\
& Z_{9}=6 \rho^{4}-6 \rho^{2}-1 \\
& Z_{10}=\rho^{3} \cos (3 \varphi) \\
& Z_{11}=\rho^{3} \sin (3 \varphi) \\
& Z_{12}=\left(4 \rho^{2}-3\right) \rho^{2} \cos (2 \varphi) \\
& Z_{13}=\left(4 \rho^{2}-3\right) \rho^{2} \sin (2 \varphi) \\
& Z_{14}=\left(10 \rho^{4}-12 \rho^{2}+3\right) \rho \cos (\varphi) \\
& Z_{15}=\left(10 \rho^{4}-12 \rho^{2}+3\right) \rho \sin (\varphi) \\
& Z_{16}=20 \rho^{6}-30 \rho^{4}+12 \rho^{2}-1 \\
& Z_{17}=\rho^{4} \cos (4 \varphi) \\
& Z_{18}=\rho^{4} \sin (4 \varphi) \\
& Z_{19}=\left(5 \rho^{2}-4\right) \rho^{3} \cos (3 \varphi) \\
& Z_{20}=\left(5 \rho^{2}-4\right) \rho^{3} \sin (3 \varphi) \\
& Z_{21}=\left(15 \rho^{4}-20 \rho^{2}+6\right) \rho^{2} \cos (2 \varphi) \\
& Z_{22}=\left(15 \rho^{4}-20 \rho^{2}+6\right) \rho^{2} \sin (2 \varphi) \\
& Z_{23}=\left(35 \rho^{6}-60 \rho^{4}+30 \rho^{2}-4\right) \rho \cos (\varphi) \\
& Z_{24}=\left(35 \rho^{6}-60 \rho^{4}+30 \rho^{2}-4\right) \rho \sin (\varphi) \\
& Z_{25}=70 \rho^{8}-140 \rho^{6}+90 \rho^{4}-20 \rho^{2}+1 \\
& Z_{26}=\rho^{5} \cos (5 \varphi) \\
& Z_{27}=\rho^{5} \sin (5 \varphi) \\
& Z_{28}=\left(6 \rho^{2}-5\right) \rho^{4} \cos (4 \varphi) \\
& Z_{29}=\left(6 \rho^{2}-5\right) \rho^{4} \sin (4 \varphi) \\
& Z_{30}=\left(21 \rho^{4}-30 \rho^{2}+10\right) \rho^{3} \cos (3 \varphi) \\
& Z_{31}=\left(21 \rho^{4}-30 \rho^{2}+10\right) \rho^{3} \sin (3 \varphi) \\
& Z_{32}=\left(56 \rho^{6}-105 \rho^{4}+60 \rho^{2}-10\right) \rho^{2} \cos (2 \varphi) \\
& Z_{33}=\left(56 \rho^{6}-105 \rho^{4}+60 \rho^{2}-10\right) \rho^{2} \sin (2 \varphi) \\
& Z_{34}=\left(126 \rho^{8}-280 \rho^{6}+210 \rho^{4}-60 \rho^{2}+5\right) \rho \cos (2 \varphi) \\
& Z_{35}=\left(126 \rho^{8}-280 \rho^{6}+210 \rho^{4}-60 \rho^{2}+5\right) \rho \sin (2 \varphi) \\
& Z_{36}=252 \rho^{10}-630 \rho^{8}+560 \rho^{6}-210 \rho^{4}+30 \rho^{2}-1 \\
&
\end{aligned}
$$




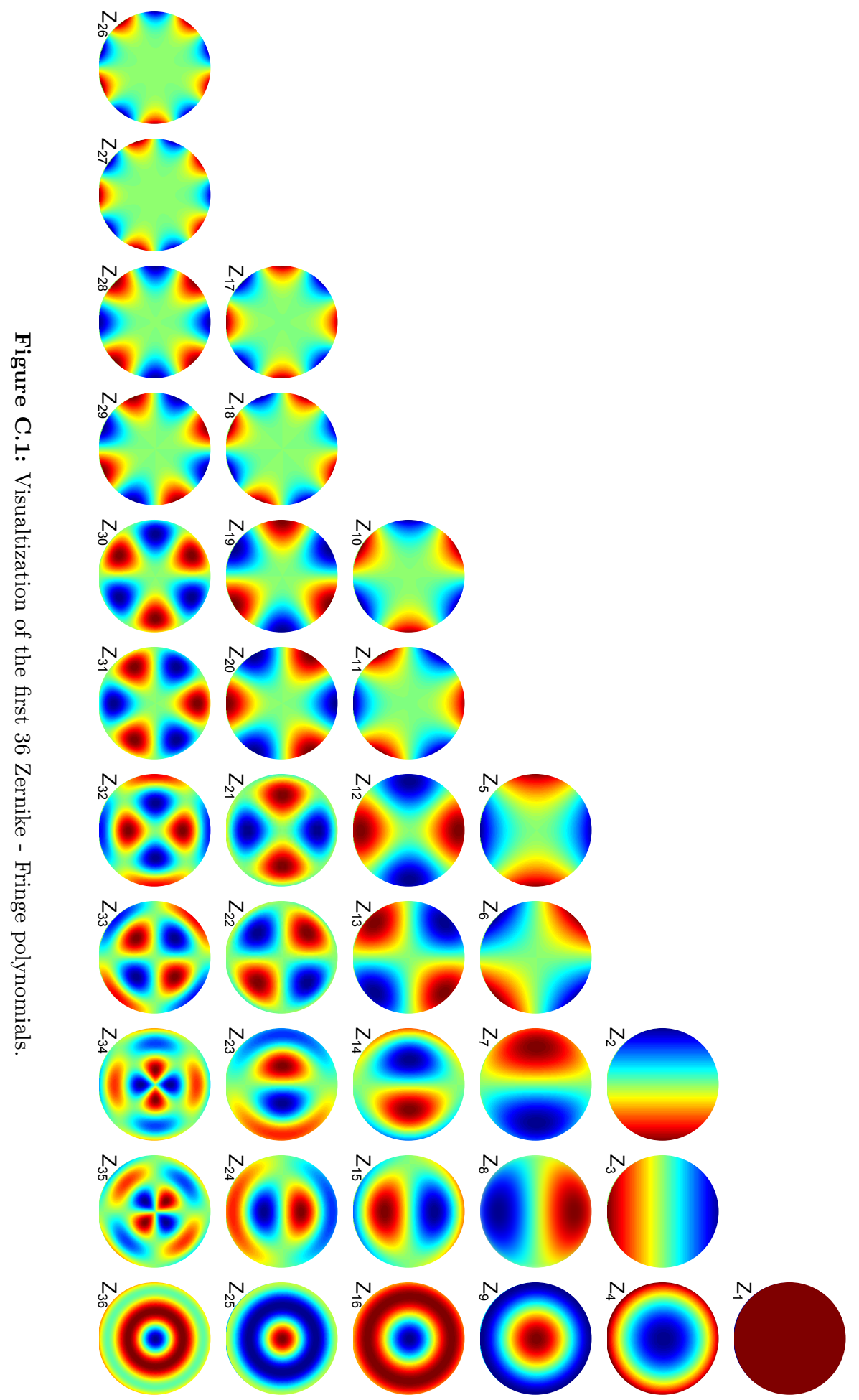




\section{Summary}

In this thesis we describe the development of a new class of optical components to enhance the imaging performance by enabling adaptations of the optics. When used at extreme ultraviolet (EUV) wavelengths, such "adaptive optics" offers the potential to achieve the highest spatial resolution in imaging known to date, i.e., a resolution down to several nanometers. These optics are discussed e.g. in the field of photolithography for the fabrication of more energy efficient and faster electronic equipment.

The topics in this thesis can be subdivided into two parts. The first part includes a mirror design that can be used to enhance the spectral quality of EUV light sources. Our design consists of a diffractive Fresnel zone plate patterned in the surface of a curved mirror to achieve spectral purification, i.e., removing undesired wavelengths of the EUV light sources. The removed light is recycled to produce more EUV light. This design achieves four orders of magnitude purification and provides a 35\% improvement in efficiency.

The second part of the thesis describes a new class of adaptive multilayer mirrors that can be used to correct wavefront distortions, reflectance degradations or wavelength deviations. These adaptive optics contain integrated crystalline piezoelectric thin films of which the film thickness can be adjusted by an externally applied voltage. Because the optics substrates typically have an amorphous structure, so far it was not possible to grow high quality crystalline piezoelectric films on them. We utilized crystalline nanosheets on these substrates to grow the highest quality, i.e., epitaxial, piezoelectric films $\left(\mathrm{PbZr}_{0.52} \mathrm{Ti}_{0.48} \mathrm{O}_{3}, \mathrm{PZT}\right)$. The PZT films show a record high piezoelectric coefficient of $98 \mathrm{pm} / \mathrm{V}$, although the piezoelectric response in this case is still reduced due to clamping by the substrate. We minimized the clamping effect by depositing films with a columnar structure, which enhances the voltage-controlled change in film thickness. In these films we measured a piezoelectric response of $280 \mathrm{pm} / \mathrm{V}$, the highest value reported on glass substrates. Importantly, the developed films can produce a stroke of $25 \mathrm{~nm}$. This order of magnitude is useful when considering to correct EUV wavefront distortions. 



\section{Nederlandse samenvatting}

In dit proefschrift beschrijven wij de ontwikkeling van een nieuwe klasse optische componenten die de beeldvorming verbeteren door middel van aanpasbare optiek. Toegepast bij extreem ultraviolette (EUV) golflengten biedt zulke "adaptieve optiek" de mogelijkheid tot het behalen van de hoogste ruimtelijke resolutie in beeldvorming tot nog toe: een resolutie tot op enkele nanometers. Het wordt verwacht dat deze optiek gebruikt wordt bij fotolithografie voor de fabricatie van energie-efficiëntere en snellere elektronische apparatuur.

De onderwerpen in dit proefschrift kunnen worden onderverdeeld in twee delen. Het eerste deel bevat een spiegelontwerp dat gebruikt kan worden om de spectrale kwaliteit van EUV-lichtbronnen te verhogen. Ons ontwerp bestaat uit een diffractieve Fresnel-zoneplaat ingebed in het oppervlak van een gebogen spiegel om spectrale zuivering te bereiken: het verwijderen van ongewenste golflengten uit de EUV-lichtbronnen. Het verwijderde licht wordt hergebruikt om meer EUVlicht te produceren. Dit ontwerp bereikt een zuivering van vier ordegrootten en biedt een $35 \%$ toename in efficiëntie.

Het tweede deel van dit proefschrift beschrijft een nieuwe klasse van adaptieve multilaagspiegels die gebruikt kunnen worden voor het corrigeren van golffrontvervormingen, reflectiviteitsdegradaties, of golflengteafwijkingen. Deze adaptieve optische componenten bevatten geïntegreerde kristallijne piëzo-elektrische dunne films waarvan de filmdikte gevarieerd kan worden door het aanbrengen van een externe spanning. Omdat optische substraten doorgaans een amorfe structuur hebben was het tot op heden niet mogelijk om hier hoogwaardige kristallijne piëzo-elektrische films op te groeien. Wij hebben kristallijne nanolagen toegepast op deze substraten om piëzo-elektrische films $\left(\mathrm{PbZr}_{0.52} \mathrm{Ti}_{0.48} \mathrm{O}_{3}, \mathrm{PZT}\right)$ van de hoogst mogelijke kwaliteit, epitaxiaal, te groeien. De PZT-films vertonen een recordhoge piëzo-elektrische coëfficiënt van $98 \mathrm{pm} / \mathrm{V}$, hoewel de piëzo-elektrische respons in dit geval nog steeds beperkt is door inklemming door het substraat. Wij hebben het effect van de inklemming geminimaliseerd door het deponeren van films met een kolomstructuur, die de verandering in filmdikte ten gevolge van een externe spanning vergroot. In deze films hebben wij een piëzo-elektrische respons gemeten van $280 \mathrm{pm} / \mathrm{V}$, de hoogst gerapporteerde waarde op glazen substraten. Nog belangrijker is dat de ontwikkelde films een slag van $25 \mathrm{~nm}$ kunnen produceren, wat voldoende groot is voor het corrigeren van de verwachte EUVgolffrontvervormingen. 



\section{Acknowledgements}

Four years of doctoral research is a long journey that is unthinkable without support from many people, my research was not an exception. Success of my research benefited greatly from encouragement, guidance and work of my supervisors, colleagues and friends in addition to my efforts. I would like to use this opportunity to thank them.

First of all, I would like to express my deep gratitude to my promotor Fred Bijkerk for his encouragement and guidance both in research and in more general sense. Your knowledge, experience and network helped me a lot towards what we achieved in this project. I should extend my deep gratitude to my second promotor Klaus Boller for his inspiring approach and invaluable contribution to my research. Fred and Klaus, during the course of my doctoral research, I experienced how different approaches to a challenge can be combined to achieve a better result. Thanks for giving me the opportunity to gain this experience that will be a lesson for me in the rest of my life.

My research project had a substantial material science part in addition to optics. Considering my background in optics and electronics, I needed to learn materials science from the very basics. I would like to gratefully acknowledge the supervision and facilities provided by Guus Rijnders in the material science part of this project.

I wish to convey my sincere thanks to Chris Lee for his inspiring daily supervision at the beginning of my Ph.D. I would like to thank Chris and also the Lee family who helped me to settle down when I first moved to Netherlands. I would like to extend my thanks to Fred van Goor for his supervision in the third year of my research. His experience and guidance helped me to broaden my knowledge.

This research would not have been possible without the input from Anuj Chopra and Werner Wessels. With Anuj we worked in close collaboration to prepare the samples. As a postdoctoral researcher you were always generous to share your experience and knowledge in material science. Thanks for your scientific input as well as nice talks we had on variety of topics like world politics. We worked with Werner when he was doing his masters assignment to prepare the first samples in the project. Thanks for your contributions to make a good start to the project. I would like to thank Minh Nguyen for his valuable contribution to this project by platinum coating the samples though he had no obligations.

I want to convey my gratefulness to my graduation committee members Guus Rijnders, Dirk Gravesteijn, Beatriz Noheda, Carsten Fallnich and Leon Abelmann for reading my thesis and providing valuable feedback.

I'm deeply thankful to Stephan Müllender, Udo Dinger, Gisela von Blanckenhagen, Toralf Grüner and Kerstin Hild from Carl-Zeiss SMT for our valuable discussions during the project meetings and their confidence in the success of 
this project. I should specifically mention and thank for the contribution of Udo during the patent writing process. Also Gisela for throughly reading every document I sent to her for clearance and speeding up the clearance process at the most needed moment.

I would like to thank the members of my research group, Laser Physics and Nonlinear Optics (LPNO), for the nice and friendly working atmosphere. I thank to Jonathan Barreaux, Jörn Epping, Youwen Fan, Marco Garcia Porcel, Siew Jean Goh, Kasper Orsel, Caterina Taddei, Yin Tao, Tom Wolterink, Ale Strooisma, Thomas Denis, Robert van der Meer, Ruud Oldenbeuving, Ingrid van der Schoor, Peter van der Slot and Bert Bastiaens for the fruitful discussions we had during the coffee breaks, Ph.D. dinners and group events.

I would like to extend my thanks to the members of our neighboring group, Complex Photonic Systems (COPS), for their great company and fruitful discussions during the coffee breaks. I thank to the members of the Inorganic Materials Science Group (IMS) for their help in practical issues while working in the labs. I would like to thank the members of the Industrial Focus Group Extreme Ultraviolet Optics (XUV) for the fruitful discussions during group meetings in the past four years and their warm welcome to my new position as a postdoctoral researcher in the group.

The doctoral life is not only about the research but also the social activities. Here I would like to thank my friends whom I played football with in various teams. I express my thanks to all the people that I was playing football in the weekends and to the Soup-a-Stars team. I would like to acknowledge my thanks to the players of the TUFAT team: Osman, Mihai, Yakup abi, Sertan, Haktan, Çağrı, Devrim, Aykan, Okan, Erdi, Koray and Can. Of course, I should mention and thank to the members of my first team, Drienerlo Z3, that I was playing in the regional league for the last four years. Sertan, Emilio, Mark, Erwin, Gerard, Roger and Joris; thank you guys for the good games and the joyful team atmosphere. Well, we did not win all the games but we had great time.

I wish to thank to the Turkish community starting from the board members of the Turkish Student Association at Twente (TUSAT). It was a great pleasure to work with Yakup, Hasan, Umut, Uğur, Çăgrı, Akın and Aykan in the TUSAT board. I would like to thank my friends Didem, Semih, Sinem, Ramazan, Kardelen, Oğuzcan, Özlem K., Engin, Özlem S., Hayrettin, Recep, Burcu, Alim, Bedile, Namı, Buğra, Aysun, Emre, Eda, Gülistan and also the people who left Enschede. We had great time both during the lunches and in many events.

Approaching to the end, I would like to express my deepest gratitude to my family. I always felt your support, trust and confidence in me. Without your support, I would not be the person I am today.

Last but not the least, I would like to heartily thank Nurcan who is the best surprise in my life. You entered my life at a surprising time and place, and made my life better in many ways with your love. From the very beginning, your understanding, support and hope made our long distance relationship and my Ph.D. life much easier. 